\title{
Dispersive Limit of the Euler-Poisson System in Higher Dimensions
}

\author{
Xueke $\mathrm{Pu}$ \\ Department of Mathematics, Chongqing University, Chongqing 400044, P.R.China; \\ Mathematical Sciences Research Institute in Chongqing \\ xuekepu@cqu.edu.cn
}

\begin{abstract}
In this paper, we consider the dispersive limit of the Euler-Poisson system for ion-acoustic waves. We establish that under the Gardner-Morikawa type transformations, the solutions of the Euler-Poisson system converge globally to the Kadomtsev-Petviashvili II equation in $\mathbb{R}^{2}$ and the Zakharov-Kuznetsov equation in $\mathbb{R}^{3}$ for well-prepared initial data, under different scalings. This justifies rigorously the KP-II limit and the ZKE limit of the Euler-Poisson equation.
\end{abstract}

AMS Subject Classification: 35Q53; 35Q35

Key Words: Euler-Poisson equation; Kadomtsev-Petviashvili-II equation; ZakharovKuznetsov equation; long wavelength limit

\section{Introduction}

Consider the Euler-Poisson (EP) system in 2D,

$$
\left\{\begin{array}{l}
\partial_{t} n+\nabla \cdot(n \mathbf{u})=0, \\
\partial_{t} \mathbf{u}+\mathbf{u} \cdot \nabla \mathbf{u}+T_{i} \frac{\nabla n}{n}=-\nabla \phi, \\
\Delta \phi=e^{\phi}-n,
\end{array}\right.
$$

where $n(t, x), \mathbf{u}(t, x)=\left(u_{1}(t, x), u_{2}(t, x)\right)$ and $\phi(t, x)$ are respectively the density, velocity of the ions and the electric potential at time $t \geq 0$ and position $x=\left(x_{1}, x_{2}\right) \in \mathbb{R}^{2}$. Here $T_{i} \geq 0$ denotes the ion temperature, and all the other physical parameters are set to be 1 .

The Euler-Poisson system (1.1) is a fundamental two-fluid model describing the dynamics of a plasma, in which compressible ion and electron fluids interact with their self-consistent electrostatic force. Here, the hot isothermal electrons are described by the Boltzmann distribution. Such an Euler-Poisson system was widely investigated in the past years. The interested readers may refer to [3, 4, 6, 10, 16, 18, 19] and the references therein. Many important nonlinear dispersive PDEs, such as the Kadomtsev-Petviashvili II (KP-II) equations [13] and the Zakharov-Kuznetsov equations (ZKE) [23] can be formally derived from the Euler-Poisson system, and they are widely used as approximate models of the Euler-Poisson system in some limit sense, in many physical contexts. These equations are higher dimensional generalizations of the Korteweg-de Vries (KdV) equation [15] and were extensively studied in the past decades. In particular, the KP-II equation describes the propagation of long nonlinear waves along the $x_{1}$-axis on the surface of a media when the variation along the $x_{2}$-axis proceeds slowly. However, there is up to now no rigorous mathematical justifications of such dispersive limits. The purpose of this paper is to justify rigorously 
these formal limits at least for well-prepared initial data. We also remark that in recent years, the KdV and the KP-I limits are justified from different interesting models, such as the water wave problem, the Schrödinger equation and the Euler-Poisson equation [2, 10, 21].

This paper is organized as follows. In Section 2, we formally derive the 2D KP-II and state the main Theorem 2.1. For clarity, the formal derivation of the ZKE from a slightly different 3D Euler-Poisson system B.1 is postponed to Appendix B. To give a unified treatment for the KP-II limit and the ZKE limit, we write the remainder equations into a unified system 2.28) by introducing some new differential notations. Section 3 and 4 are dedicated to the proof of Theorem 2.1. In Section 3, we prove Theorem 2.1 for the case of $T_{i}>0$ for both the KP-II limit in 2D and the ZKE limit in 3D. In Section 4, we prove Theorem 2.1 for the case of $T_{i}=0$ for the ZKE limit in 3D.

Throughout this paper, we use $[A, B]=A B-B A$ to denote the commutator of $A$ and $B$ and $\|\cdot\|_{X}$ to denote an $X$-norm. When $X=L^{2}$, the subscript of $\|\cdot\|_{L^{2}}$ is usually omitted. We also use $\langle f, g\rangle_{0}=\int f g d x$ to denote the inner product of two $L^{2}$ functions.

\section{Formal derivation and the main results}

\subsection{Formal derivation of the KPE}

In this subsection, we derive the KP equation from the 2D Euler-Poisson equation 1.1). Consider the following Gardner-Morikawa type of transformation in (1.1)

$$
\varepsilon^{1 / 2}\left(x_{1}-V t\right) \rightarrow x_{1}, \quad \varepsilon x_{2} \rightarrow x_{2}, \quad \varepsilon^{3 / 2} t \rightarrow t
$$

where $\varepsilon$ stands for the amplitude of the initial disturbance and is assumed to be small compared with unity and $V$ is the wave speed to be determined. Then we obtain the parameterized system

$$
\left\{\begin{array}{l}
\varepsilon \partial_{t} n-V \partial_{x_{1}} n+\partial_{x_{1}}\left(n u_{1}\right)+\varepsilon^{1 / 2} \partial_{x_{2}}\left(n u_{2}\right)=0 \\
\varepsilon \partial_{t} u_{1}-V \partial_{x_{1}} u_{1}+u_{1} \partial_{x_{1}} u_{1}+\varepsilon^{1 / 2} u_{2} \partial_{x_{2}} u_{1}+T_{i} \frac{\partial_{x_{1}} n}{n}=-\partial_{x_{1}} \phi \\
\varepsilon \partial_{t} u_{2}-V \partial_{x_{1}} u_{2}+u_{1} \partial_{x_{1}} u_{2}+\varepsilon^{1 / 2} u_{2} \partial_{x_{2}} u_{2}+T_{i} \frac{\varepsilon^{1 / 2} \partial_{x_{2}} n}{n}=-\varepsilon^{1 / 2} \partial_{x_{2}} \phi \\
\varepsilon \partial_{x_{1}}^{2} \phi+\varepsilon^{2} \partial_{x_{2}}^{2} \phi=e^{\phi}-n
\end{array}\right.
$$

Consider the following formal expansion

$$
\left\{\begin{array}{l}
n=1+\varepsilon n^{(1)}+\varepsilon^{2} n^{(2)}+\varepsilon^{3} n^{(3)}+\cdots \\
u_{1}=\varepsilon u_{1}^{(1)}+\varepsilon^{2} u_{1}^{(2)}+\varepsilon^{3} u_{1}^{(3)}+\cdots \\
u_{2}=\varepsilon^{3 / 2} u_{2}^{(1)}+\varepsilon^{5 / 2} u_{2}^{(2)}+\varepsilon^{7 / 2} u_{2}^{(3)}+\cdots \\
\phi=\varepsilon \phi^{(1)}+\varepsilon^{2} \phi^{(2)}+\varepsilon^{3} \phi^{(3)}+\cdots
\end{array}\right.
$$

Plugging this formal expansion into the system 2.2), we get a power series of $\varepsilon$, whose coefficients depend on $\left(n^{(k)}, \mathbf{u}^{(k)}, \phi^{(k)}\right)$ for $k \geq 1$, where $\mathbf{u}^{(k)}=\left(u_{1}^{(k)}, u_{2}^{(k)}\right)^{T}$.

\subsubsection{Derivation of the KPE for $n^{(1)}$}

From the power series of $\varepsilon$ we thus obtained, we get at the order of $\varepsilon$ :

Coefficients of $\varepsilon^{1}$ :

$$
\left\{\begin{array}{l}
-V \partial_{x_{1}} n^{(1)}+\partial_{x_{1}} u_{1}^{(1)}=0 \\
-V \partial_{x_{1}} u_{1}^{(1)}+T_{i} \partial_{x_{1}} n^{(1)}=-\partial_{x_{1}} \phi^{(1)} \\
0=\phi^{(1)}-n^{(1)}
\end{array}\right.
$$


To get a nontrivial solution of $\left(n^{(1)}, u_{1}^{(1)}, \phi^{(1)}\right)$, we let the determinant of the coefficient matrix of (2.4) to vanish to obtain

$$
V^{2}=T_{i}+1
$$

For definiteness, we set $V=\sqrt{T_{i}+1}$ in the following.

At higher orders, we obtain

Coefficients of $\varepsilon^{3 / 2}$ :

$$
-V \partial_{x_{1}} u_{2}^{(1)}+T_{i} \partial_{x_{2}} n^{(1)}=-\partial_{x_{2}} \phi^{(1)}
$$

Coefficients of $\varepsilon^{2}$ :

$$
\left\{\begin{array}{l}
\partial_{t} n^{(1)}-V \partial_{x_{1}} n^{(2)}+\partial_{x_{1}} u_{1}^{(2)}+\partial_{x_{1}}\left(n^{(1)} u_{1}^{(1)}\right)+\partial_{x_{2}} u_{2}^{(1)}=0 \\
\partial_{t} u_{1}^{(1)}-V \partial_{x_{1}} u_{1}^{(2)}+u_{1}^{(1)} \partial_{x_{1}} u_{1}^{(1)}+T_{i}\left\{\partial_{x_{1}} n^{(2)}-n^{(1)} \partial_{x_{1}} n^{(1)}\right\}=-\partial_{x_{1}} \phi^{(1)} \\
\partial_{x_{1}}^{2} \phi^{(1)}=\phi^{(2)}+\frac{1}{2}\left(\phi^{(1)}\right)^{2}-n^{(2)}
\end{array}\right.
$$

From (2.4), we may assume that

$$
u_{1}^{(1)}=V n^{(1)}, \phi^{(1)}=n^{(1)},
$$

which also make (2.4) valid, thanks to (2.5). Then from (2.6), we have

$$
\partial_{x_{1}} u_{2}^{(1)}=V \partial_{x_{2}} n^{(1)}
$$

thanks to 2.8). Therefore, to solve $n^{(1)}, \mathbf{u}^{(1)}$ and $\phi^{(1)}$, we need only to solve $n^{(1)}$.

To find out the equation satisfied by $n^{(1)}$, we take $\partial_{x_{1}}$ of $(2.7 \mathrm{c})$, multiply $(2.7 \mathrm{a})$ by $V$, and then add them to $2.7 \mathrm{~b}$. We obtain

$$
\partial_{t} n^{(1)}+V n^{(1)} \partial_{x_{1}} n^{(1)}+\frac{1}{2 V} \partial_{x_{1}}^{3} n^{(1)}+\frac{1}{2} \partial_{x_{2}} u_{2}^{(1)}=0 .
$$

Differentiating this equation with respect to $x_{1}$, and using [2.9], we obtain

$$
\partial_{x_{1}}\left\{\partial_{t} n^{(1)}+V n^{(1)} \partial_{x_{1}} n^{(1)}+\frac{1}{2 V} \partial_{x_{1}}^{3} n^{(1)}\right\}+\frac{V}{2} \partial_{x_{2}}^{2} n^{(1)}=0 .
$$

This is the Kadomtsev-Petviashvili II equation satisfied by the first order profile $n^{(1)}$.

Proposition 2.1. The Cauchy problem for the KP-II equation is well-posed in $H^{s}\left(\mathbb{R}^{2}\right)$ for $s \geq 0$.

This theorem is proved in the seminal paper of Bourgain [1]. Actually, it is known to be well-posed in spaces of much lower regularity [11, 12, 20]. However, Proposition 2.1] is enough for our purpose.

Remark 2.1. The system of 2.8), 2.9) and (2.11) is a closed system. Once $n^{(1)}$ is solved from 2.11), we have all the other first order profiles $\mathbf{u}^{(1)}$ and $\phi^{(1)}$ from (2.8) and (2.9).

From (2.7a) and (2.7c), we may assume

$$
\left\{\begin{array}{l}
u_{1}^{(2)}=V n^{(2)}+\underline{u}_{1}^{(1)} K P \\
\phi^{(2)}=n^{(2)}+\underline{\phi}_{K P}^{(1)},
\end{array}\right.
$$

where $\underline{u}_{1}^{(1)}$ and $\underline{\phi}_{K P}^{(1)}$ depend only on $n^{(1)}$, which is smooth in some time interval $\left[0, \tau_{*}\right)$ thanks to Proposition 2.1. 
At the order $\varepsilon^{5 / 2}$, we obtain

Coefficients of $\varepsilon^{5 / 2}$ :

$$
-V \partial_{x_{1}} u_{2}^{(2)}+u_{1}^{(1)} \partial_{x_{1}} u_{2}^{(1)}+T_{i}\left\{\partial_{x_{2}} n^{(2)}-n^{(1)} \partial_{x_{2}} n^{(1)}\right\}=-\partial_{x_{2}} \phi^{(2)}
$$

By using (2.12) and rearranging, we have

$$
V \partial_{x_{1}} u_{2}^{(2)}=\left(T_{i}+1\right) \partial_{x_{2}} n^{(2)}+u_{1}^{(1)} \partial_{x_{1}} u_{2}^{(1)}-T_{i} n^{(1)} \partial_{x_{2}} n^{(1)}+\partial_{x_{2}} \underline{\phi}_{K P}^{(1)}
$$

\subsubsection{Derivation of the Linearized KPE for $n^{(k)}$}

From (2.12) and (2.14), we see that to determine $\left(n^{(2)}, \mathbf{u}^{(2)}, \phi^{(2)}\right)$, we need only to determine $n^{(2)}$.

At the order of $\varepsilon^{3}$, we obtain

Coefficients of $\varepsilon^{3}$ :

$$
\left\{\begin{array}{c}
\partial_{t} n^{(2)}-V \partial_{x_{1}} n^{(3)}+\partial_{x_{1}} u_{1}^{(3)} \\
\quad+\partial_{x_{1}}\left(n^{(1)} u_{1}^{(2)}+n^{(2)} u_{1}^{(1)}\right)+\partial_{x_{2}}\left(u_{2}^{(2)}+n^{(1)} u_{2}^{(1)}\right)=0, \\
\partial_{t} u_{1}^{(2)}-V \partial_{x_{1}} u_{1}^{(3)}+\partial_{x_{1}}\left(u_{1}^{(1)} u_{1}^{(2)}\right)+T_{i} \partial_{x_{1}} n^{(3)} \\
\quad+T_{i}\left\{\partial_{x_{1}} n^{(1)}\left(\frac{1}{2}\left(n^{(1)}\right)^{2}-n^{(2)}\right)-\partial_{x_{1}} n^{(2)} n^{(1)}\right\}=-\partial_{x_{1}} \phi^{(3)}, \\
\partial_{x_{1}}^{2} \phi^{(2)}+\partial_{x_{2}}^{2} \phi^{(1)}=\phi^{(3)}+\phi^{(1)} \phi^{(2)}+\frac{1}{3 !}\left(\phi^{(1)}\right)^{3}-n^{(3)} .
\end{array}\right.
$$

Taking $\partial_{x_{1}}$ of (2.15c), multiplying (2.15a) with $V$, and then adding them to (2.15b), we obtain the linearized inhomogeneous KP equation

$$
\partial_{x_{1}}\left\{\partial_{t} n^{(2)}+2 V \partial_{x_{1}}\left(n^{(1)} n^{(2)}\right)+\frac{1}{2 V} \partial_{x_{1}}^{3} n^{(2)}\right\}+\frac{V}{2} \partial_{x_{2}}^{2} n^{(2)}=\underline{G}_{K P}^{(1)},
$$

where we have used (2.12) and (2.14). Here $\underline{G}_{K P}^{(1)}$ depends only on $n^{(1)}$ and comes form the inhomogeneous dependence of $u_{1}^{(2)}$ and $\phi^{(2)}$ on $n^{(2)}$ in 2.12).

At the order $\varepsilon^{7 / 2}$, we obtain Coefficients of $\varepsilon^{7 / 2}$ :

$$
\begin{aligned}
\partial_{t} u_{2}^{(2)}- & V \partial_{x_{1}} u_{2}^{(3)}+u_{1}^{(1)} \partial_{x_{1}} u_{2}^{(2)}+u_{1}^{(2)} \partial_{x_{1}} u_{2}^{(1)}+u_{2}^{(1)} \partial_{x_{2}} u_{2}^{(1)}+T_{i} \partial_{x_{1}} n^{(3)} \\
+ & T_{i}\left\{\partial_{x_{2}} n^{(1)}\left(\frac{1}{2}\left(n^{(1)}\right)^{2}-n^{(2)}\right)-\partial_{x_{2}} n^{(2)} n^{(1)}\right\}=-\partial_{x_{2}} \phi^{(3)}
\end{aligned}
$$

Inductively, we can derive all the profiles $\left(n^{(k)}, \mathbf{u}^{(k)}, \phi^{(k)}\right)$ for $k \geq 3$. Proceeding as above, we obtain the following linearized inhomogeneous KP equation for $n^{(k)}$ for $k \geq 3$ :

$$
\partial_{x_{1}}\left\{\partial_{t} n^{(k)}+2 V \partial_{x_{1}}\left(n^{(1)} n^{(k)}\right)+\frac{1}{2 V} \partial_{x_{1}}^{3} n^{(k)}\right\}+\frac{V}{2} \partial_{x_{2}}^{2} n^{(k)}=\underline{G}_{K P}^{(k-1)}
$$

where the inhomogeneous term $\underline{G}_{K P}^{(k-1)}$ depends only on $\left(n^{(j)}, \mathbf{u}^{(j)}, \phi^{(j)}\right)$ for $1 \leq j \leq k-1$.

Since (2.18) is linear in $n^{(k)}$, we easily obtain

Proposition 2.2. The Cauchy problem of the linearized inhomogeneous KPE $(2.18)(k \geq 2)$ is well-posed in $H^{s}\left(\mathbb{R}^{3}\right)$ for $s \geq 0$. 


\subsection{Remainder system}

To make the above procedure rigorous, we need to cut off and consider the remainder terms. For this, we consider the following expansion

$$
\left\{\begin{array}{l}
n=1+\varepsilon n^{(1)}+\varepsilon^{2} n^{(2)}+\varepsilon^{3} n^{(3)}+\varepsilon^{2} n_{R}^{\varepsilon} \\
u_{1}=\varepsilon u_{1}^{(1)}+\varepsilon^{2} u_{1}^{(2)}+\varepsilon^{3} u_{1}^{(3)}+\varepsilon^{2} u_{1}^{\varepsilon}, \\
u_{2}=\varepsilon^{3 / 2} u_{2}^{(1)}+\varepsilon^{5 / 2} u_{2}^{(2)}+\varepsilon^{7 / 2} u_{2}^{(3)}+\varepsilon^{2} u_{2}^{\varepsilon} \\
\phi=\varepsilon \phi^{(1)}+\varepsilon^{2} \phi^{(2)}+\varepsilon^{3} \phi^{(3)}+\varepsilon^{2} \phi_{R}^{\varepsilon}
\end{array}\right.
$$

where $\left(n^{(i)}, \mathbf{u}^{(i)}, \phi^{(i)}\right)$ for $1 \leq i \leq 3$ are the first few profiles constructed above and $\left(n_{R}^{\varepsilon}, \mathbf{u}_{R}^{\varepsilon}, \phi_{R}^{\varepsilon}\right)$ are the remainder terms that may depend on $\varepsilon$ and $\left(n^{(i)}, \mathbf{u}^{(i)}, \phi^{(i)}\right)$ for $1 \leq i \leq 3$. More precisely, $n^{(1)}$ satisfies (2.11), $u_{1}^{(1)}, \phi^{(1)}$ satisfy (2.8) and $u_{2}^{(1)}$ satisfies (2.9); $n^{(2)}$ satisfies (2.16), $u_{1}^{(2)}, \phi^{(2)}$ satisfy (2.12) and $u_{2}^{(2)}$ satisfies 2.14; ; and similarly for the third order profile $\left(n^{(3)}, \mathbf{u}^{(3)}, \phi^{3}\right)$.

Inserting (2.19) into (2.2), and then subtracting the systems of the coefficient up to order $\varepsilon^{7 / 2}$, we obtain the remainder system of $\left(n_{R}^{\varepsilon}, \mathbf{u}_{R}^{\varepsilon}, \phi_{R}^{\varepsilon}\right)$. For notational convenience, we denote $\mathbf{u}=\left(u_{1}, u_{2}\right)^{T}, \widetilde{\mathbf{u}}=$ $\left(\widetilde{u}_{1}, \widetilde{u}_{2}\right)^{T}, \mathbf{u}_{R}^{\varepsilon}=\left(u_{1}^{\varepsilon}, u_{2}^{\varepsilon}\right)^{T}$ and

$$
\begin{aligned}
& \widetilde{n}=n^{(1)}+\varepsilon n^{(2)}+\varepsilon^{2} n^{(3)}, \quad \widetilde{\phi}=\phi^{(1)}+\varepsilon \phi^{(2)}+\varepsilon^{2} \phi^{(3)} \\
& \widetilde{u}_{1}=u_{1}^{(1)}+\varepsilon u_{1}^{(2)}+\varepsilon^{2} u_{1}^{(3)}, \quad \widetilde{u}_{2}=\varepsilon^{1 / 2} u_{2}^{(1)}+\varepsilon^{3 / 2} u_{2}^{(2)}+\varepsilon^{5 / 2} u_{2}^{(3)} .
\end{aligned}
$$

Proposition 2.3. Let $(n, \boldsymbol{u}, \phi)$ in (2.19) be a solution of the Euler-Poisson equation (1.1), then the remain$\operatorname{ders} n_{R}^{\varepsilon}, \boldsymbol{u}_{R}^{\varepsilon}$ and $\phi_{R}^{\varepsilon}$ in (2.19) satisfy

$$
\left\{\begin{aligned}
\partial_{t} n_{R}^{\varepsilon}- & \frac{V-u_{1}}{\varepsilon} \partial_{x_{1}} n_{R}^{\varepsilon}+\frac{\varepsilon^{1 / 2} u_{2}}{\varepsilon} \partial_{x_{2}} n_{R}^{\varepsilon}+\frac{n}{\varepsilon} \partial_{x_{1}} u_{1}^{\varepsilon}+\frac{\varepsilon^{1 / 2} n}{\varepsilon} \partial_{x_{2}} u_{2}^{\varepsilon} \\
& +n_{R}^{\varepsilon} \partial_{x_{1}} \widetilde{u}_{1}+\varepsilon^{1 / 2} n_{R}^{\varepsilon} \partial_{x_{2}} \widetilde{u}_{2}+u_{1}^{\varepsilon} \partial_{x_{1}} \widetilde{n}+\varepsilon^{1 / 2} u_{2}^{\varepsilon} \partial_{x_{2}} \widetilde{n}+\varepsilon R_{n}=0, \\
\partial_{t} u_{1}^{\varepsilon} & -\frac{V-u_{1}}{\varepsilon} \partial_{x_{1}} u_{1 R}^{\varepsilon}+\frac{\varepsilon^{1 / 2} u_{2}}{\varepsilon} \partial_{x_{2}} u_{1}^{\varepsilon}+u_{1}^{\varepsilon} \partial_{x_{1}} \widetilde{u}_{1}+\varepsilon^{1 / 2} u_{2}^{\varepsilon} \partial_{x_{2}} \widetilde{u}_{1} \\
& +T_{i} \frac{\partial_{x_{1}} n_{R}^{\varepsilon}}{\varepsilon n}-T_{i} \frac{p_{1}}{n} n_{R}^{\varepsilon}-T_{i} \frac{\varepsilon R_{T 1}}{n}+\varepsilon R_{1}=-\frac{1}{\varepsilon} \partial_{x_{1}} \phi_{R}^{\varepsilon}, \\
\partial_{t} u_{2}^{\varepsilon} & -\frac{V-u_{1}}{\varepsilon} \partial_{x_{1}} u_{2}^{\varepsilon}+\frac{\varepsilon^{1 / 2} u_{2}}{\varepsilon} \partial_{x_{2}} u_{2}^{\varepsilon}+u_{1}^{\varepsilon} \partial_{x_{1}} \widetilde{u}_{2}+\varepsilon^{1 / 2} u_{2}^{\varepsilon} \partial_{x_{2}} \widetilde{u}_{2} \\
& +T_{i} \frac{\varepsilon^{1 / 2} \partial_{x_{2}} n_{R}^{\varepsilon}}{\varepsilon n}-T_{i} \frac{\varepsilon^{1 / 2} p_{2}}{n} n_{R}^{\varepsilon}-T_{i} \frac{\varepsilon^{1 / 2} \varepsilon R_{T 2}}{n}+\varepsilon R_{2}=-\frac{\varepsilon^{1 / 2}}{\varepsilon} \partial_{x_{2}} \phi_{R}^{\varepsilon}, \\
\varepsilon \partial_{x_{1}}^{2} \phi_{R}^{\varepsilon} & +\varepsilon^{2} \partial_{x_{2}}^{2} \phi_{R}^{\varepsilon}=\phi_{R}^{\varepsilon}-n_{R}^{\varepsilon}+\varepsilon \phi^{(1)} \phi_{R}^{\varepsilon}+\varepsilon^{3 / 2} R_{\phi},
\end{aligned}\right.
$$

where $\left(\widetilde{n}, \widetilde{u}_{1}, \widetilde{u}_{2}\right)$ are given in (2.20) and $\left(n^{(i)}, u_{1}{ }^{(i)}, u_{2}{ }^{(i)}, \phi^{(i)}\right)$ for $1 \leq i \leq 3$ satisfy the systems (2.4), (2.6), (2.7), (2.13), 2.15) and (2.17). Here, $R_{n}, \boldsymbol{R}_{\boldsymbol{u}}=\left(R_{1}, R_{2}\right)$ and the coefficients $p_{i}$ and $R_{T i}$ for $i=1,2$ depend only on $\left(\widetilde{n}, \widetilde{u}_{1}, \widetilde{u}_{2}, \widetilde{\phi}\right)$. In $(2.21 \mathrm{a}), \boldsymbol{e}_{1}=(1,0,0)^{\prime}$ is a constant vector.

We also give some basic estimates for the remainder term $R_{\phi}$ in the following

Lemma 2.1. Let $k \geq 0$ be an integer, then there exists a constant $1 \leq C_{1}=C_{1}\left(\sqrt{\varepsilon}\left\|\phi_{R}^{\varepsilon}\right\|_{H^{\delta}}\right)$, such that

$$
\begin{aligned}
\left\|R_{\phi}\right\|_{H^{k}} & \leq C_{1}\left(\sqrt{\varepsilon}\left\|\phi_{R}^{\varepsilon}\right\|_{H^{\delta}}\right)\left(1+\left\|\phi_{R}^{\varepsilon}\right\|_{H^{k}}\right), \quad \text { and } \\
\left\|\partial_{t} R_{\phi}\right\|_{H^{k}} & \leq C_{1}\left(\sqrt{\varepsilon}\left\|\phi_{R}^{\varepsilon}\right\|_{H^{\delta}}\right)\left(1+\left\|\partial_{t} \phi_{R}^{\varepsilon}\right\|_{H^{k}}\right),
\end{aligned}
$$

where $\delta=\max \{2, k-1\}$. Furthermore, the constant $C_{1}(\cdot)$ can be chosen to be nondecreasing. 
The proof of Proposition 2.3 and Lemma 2.1 is given in the Appendix A. To prove the KPE limit rigorously, we need only to derive some uniform estimates for the remainder $\left(n_{R}^{\varepsilon}, \mathbf{u}_{R}^{\varepsilon}, \phi_{R}^{\varepsilon}\right)$.

From a different scaling, we can derive the Zakharov-Kuznetsov equation from the Euler-Poisson system with static magnetic field. The derivations of such a ZKE and its remainder equation are detailed in Appendix B. See Proposition B.3

\subsection{Unified remainder system}

We introduce a unified form the remainder system 2.21) for the KP-II limit in 2D and B.25) for the ZKE limit in 3D. This will substantially simplify the presentation of this paper. For this purpose, we define

$$
\bar{\nabla}= \begin{cases}\left(\partial_{x_{1}}, \sqrt{\varepsilon} \partial_{x_{2}}\right)^{T} & d=2 \\ \left(\partial_{x_{1}}, \partial_{x_{2}}, \partial_{x_{3}}\right)^{T} & d=3\end{cases}
$$

where ${ }^{T}$ means transpose and $\bar{\Delta}=\bar{\nabla} \cdot \bar{\nabla}$. We also denote

$$
\mathbf{p}= \begin{cases}\left(p_{1}, \sqrt{\varepsilon} p_{2}\right)^{T} & d=2, \\ \left(p_{1}, p_{2}, p_{3}\right)^{T} & d=3,\end{cases}
$$

and

$$
\mathbf{R}_{T}= \begin{cases}\left(R_{T 1}, \sqrt{\varepsilon} R_{T 2}\right)^{T} & d=2, \\ \left(R_{T 1}, R_{T 2}, R_{T 3}\right)^{T} & d=3,\end{cases}
$$

where $p_{i}$ and $R_{T i}$ are those in $(2.21$. Let $(\widetilde{n}, \widetilde{\mathbf{u}}, \widetilde{\phi})$ denote either the expressions in 2.20 for the 2D case or the expressions in (B.24) for the 3D case. Under these notations, we have

$$
(n, \mathbf{u}, \phi)=(1+\varepsilon \widetilde{n}, \varepsilon \widetilde{\mathbf{u}}, \varepsilon \widetilde{\phi})+\left(\varepsilon^{2} n_{R}^{\varepsilon}, \varepsilon^{2} \mathbf{u}_{R}^{\varepsilon}, \varepsilon^{2} \phi_{R}^{\varepsilon}\right)
$$

for both the $2 \mathrm{D}$ case and the $3 \mathrm{D}$ case.

Proposition 2.4. Under these notations of (2.24)-(2.27), the remainder equations for (2.21) and (B.25) can be unified into

$$
\left\{\begin{array}{c}
\partial_{t} n_{R}^{\varepsilon}-\frac{V \boldsymbol{e}_{1}-\boldsymbol{u}}{\varepsilon} \cdot \bar{\nabla} n_{R}^{\varepsilon}+\frac{n}{\varepsilon} \bar{\nabla} \cdot \boldsymbol{u}_{R}^{\varepsilon}+n_{R}^{\varepsilon} \bar{\nabla} \cdot \widetilde{\boldsymbol{u}}+\boldsymbol{u}_{R}^{\varepsilon} \cdot \bar{\nabla} \widetilde{n}+\varepsilon R_{n}=0, \\
\partial_{t} \boldsymbol{u}_{R}^{\varepsilon}-\frac{V \boldsymbol{e}_{1}-\boldsymbol{u}}{\varepsilon} \cdot \bar{\nabla} \boldsymbol{u}_{R}^{\varepsilon}+\boldsymbol{u}_{R}^{\varepsilon} \cdot \bar{\nabla} \widetilde{\boldsymbol{u}}+\frac{T_{i}}{\varepsilon n} \bar{\nabla} n_{R}^{\varepsilon} \\
\quad-\frac{T_{i} \boldsymbol{p}}{\varepsilon n} n_{R}^{\varepsilon}+\varepsilon \boldsymbol{R}_{\boldsymbol{u}}-\frac{T_{i} \varepsilon}{n} \boldsymbol{R}_{T}=-\frac{1}{\varepsilon} \bar{\nabla} \phi_{R}^{\varepsilon}+\frac{b}{\varepsilon^{3 / 2}} \boldsymbol{u}_{R}^{\varepsilon} \times \boldsymbol{e}_{1}, \\
\varepsilon \bar{\Delta} \phi_{R}^{\varepsilon}=\phi_{R}^{\varepsilon}-n_{R}^{\varepsilon}+\varepsilon \phi^{(1)} \phi_{R}^{\varepsilon}+\varepsilon^{3 / 2} R_{\phi},
\end{array}\right.
$$

where the constant $b=0$ in $2 D$ and $b=1$ in $3 D$ to indicate the presence of a static magnetic field. Furthermore, 2.28c is equivalent to the following

$$
\varepsilon \bar{\Delta} \phi_{R}^{\varepsilon}=\phi_{R}^{\varepsilon}-n_{R}^{\varepsilon}+\varepsilon \phi^{(1)} \phi_{R}^{\varepsilon}+\frac{\varepsilon^{2}}{2}\left(\phi_{R}^{\varepsilon}\right)^{2}+\varepsilon^{2} \bar{R}_{\phi} .
$$

Here, $R_{\phi}$ and $\bar{R}_{\phi}$ satisfy the estimates in Lemma 2.1

From Proposition 2.1 and 2.2 we may assume that the known profiles $(\widetilde{n}, \widetilde{\mathbf{u}}, \widetilde{\phi})$ are smooth enough such that there exist some $C>0$ and some $s \geq 4$,

$$
\sup _{\left[0, \tau_{*}\right]}\left\|(\widetilde{n}, \widetilde{\mathbf{u}}, \widetilde{\phi}), R_{n}, \mathbf{R}_{\mathbf{u}}, \mathbf{R}_{T}\right\|_{H^{s}} \leq C,
$$

where $\tau_{*}$ is the existence time in Proposition 2.1 or Proposition (B.1). 


\subsection{Main results}

Now, we are in a good position to state the main results of this paper. We first introduce the following $\varepsilon$-dependent norms. We denote (the triple norm)

$$
\left\{\begin{array}{l}
\left\|n_{R}^{\varepsilon}\right\|_{s^{\prime}}^{2}=\left\|\mathbf{u}_{R}^{\varepsilon}\right\|_{H^{s^{\prime}}}^{2}, \\
\left\|\mathbf{u}_{R}^{\varepsilon}\right\|_{s^{\prime}}^{2}=\left\|\mathbf{u}_{R}^{\varepsilon}\right\|_{H^{s^{\prime}}}^{2}+\varepsilon\left\|\bar{\nabla} \mathbf{u}_{R}^{\varepsilon}\right\|_{H^{s^{\prime}}}^{2}, \\
\left\|\phi_{R}^{\varepsilon}\right\|_{s^{\prime}}^{2}=\left\|\phi_{R}^{\varepsilon}\right\|_{H^{s^{\prime}}}^{2}+\varepsilon\left\|\bar{\nabla} \phi_{R}^{\varepsilon}\right\|_{H^{s^{\prime}}}^{2}+\varepsilon^{2}\left\|\bar{\Delta} \phi_{R}^{\varepsilon}\right\|_{H^{s^{\prime}}}^{2},
\end{array}\right.
$$

where $\|\cdot\|_{H^{s^{\prime}}}$ is the standard Sobolev norm.

Theorem 2.1. Let $s \geq 4$ be such that (2.30) holds and $\left(n^{(i)}, \boldsymbol{u}^{(i)}, \phi^{(i)}\right) \in H^{s}$ for $1 \leq i \leq 3$ (resp. $1 \leq i \leq 6$ ) be solutions constructed on the interval $\left[0, \tau_{*}\right)$ in Proposition 2.1 (resp. Proposition B.1] with initial data $\left(n_{0}^{(i)}, \boldsymbol{u}_{0}^{(i)}, \phi_{0}^{(i)}\right) \in H^{s}$. Let $4 \leq s^{\prime} \leq s$ and assume that the initial data $\left(n_{0}, \boldsymbol{u}_{0}, \phi_{0}\right)$ for the EP system (1.1) (resp. EP system (1B.1) has the expansion of the form (2.19) (resp. (B.23) and $\left.\left(n_{R}^{\varepsilon}, u_{R}^{\varepsilon}, \phi_{R}^{\varepsilon}\right)\right|_{t=0}=$ $\left(n_{R 0}^{\varepsilon}, u_{R 0}^{\varepsilon}, \phi_{R 0}^{\varepsilon}\right)$ satisfy (2.28). Then for any $0<\tau_{0}<\tau_{*}$, there exist $\varepsilon_{0}>0$ and $C_{\tau_{0}}>0$ such that when $0<\varepsilon<\varepsilon_{0}$, the solutions of the EP system (1.1) (resp. (B.1) with initial data $\left(n_{0}, \boldsymbol{u}_{0}, \phi_{0}\right)$ can be expressed in the expansion (2.19) (resp. (B.23)), such that the solutions $\left(n_{R}^{\varepsilon}, u_{R}^{\varepsilon}, \phi_{R}^{\varepsilon}\right)$ of (2.28) satisfy

1) when $T_{i}>0$, for either $d=2$ or $d=3$,

$$
\sup _{\left[0, \tau_{0}\right]}\left\|\left(n_{R}^{\varepsilon}, \boldsymbol{u}_{R}^{\varepsilon}, \phi_{R}^{\varepsilon}\right)\right\|_{H^{s^{\prime}}}^{2} \leq C_{\tau_{0}}\left(1+\left\|\left(n_{R 0}^{\varepsilon}, u_{R 0}^{\varepsilon}, \phi_{R 0}^{\varepsilon}\right)\right\|_{H^{s^{\prime}}}^{2}\right),
$$

2) when $T_{i}=0$, for $d=3$

$$
\sup _{\left[0, \tau_{0}\right]}\left\|\left(n_{R}^{\varepsilon}, u_{R}^{\varepsilon}, \phi_{R}^{\varepsilon}\right)\right\|_{s^{\prime}}^{2} \leq C_{\tau_{0}}\left(1+\left\|\left(n_{R 0}^{\varepsilon}, u_{R 0}^{\varepsilon}, \phi_{R 0}^{\varepsilon}\right)\right\|_{s^{\prime}}^{2}\right),
$$

where $\left\|\left(n_{R}^{\varepsilon}, \boldsymbol{u}_{R}^{\varepsilon}, \phi_{R}^{\varepsilon}\right)\right\|_{s^{\prime}}^{2}=\left\|n_{R}^{\varepsilon}\right\|_{s^{\prime}}^{2}+\left\|\boldsymbol{u}_{R}^{\varepsilon}\right\|_{s^{\prime}}^{2}+\left\|\phi_{R}^{\varepsilon}\right\|_{s^{\prime}}^{2}$ is defined in 2.31).

Remark 2.2. When $T_{i}>0$, the result for the KP-II limit in 2D can be generalized to any dimensions $d \geq 2$, following the same lines of the proof in Section 3. But the index $s^{\prime}$ will be replaced by a greater one depending on the dimension $d \geq 2$.

This theorem provides a rigorous justification of the Kadomtsev-Petviashvili equation in 2D and the Zakharov-Kuznetsov equation in 3D from the Euler-Poisson system in the long wavelength limit (in the ZKE limit, the Euler-Poisson we used is $(\mathbb{B . 1}$, see Appendix B). To prove this result, we need to derive a uniform bound for the remainder $\left(n_{R}^{\varepsilon}, \mathbf{u}_{R}^{\varepsilon}, \phi_{R}^{\varepsilon}\right)$ in (2.28). However, this is not starightforward, especially when $T_{i}=0$. When $T_{i}=0$, the system of (2.28a) and (2.28b) for $\left(n_{R}^{\varepsilon}, \mathbf{u}_{R}^{\varepsilon}\right)$ does not match the common structure of Friedrich's symmetric systems. Because of this, the approach by Grenier [5] cannot be applied, which depends heavily on the symmetrizability of the underlying system. To overcome this difficulty, we need to combine the energy estimates with the delicate structure of the Poisson equation carefully. This is why we introduce the norm $\|\cdot\|_{s^{\prime}}$ in the case $T_{i}=0$.

\section{Proof of Theorem 2.1 for $T_{i}>0$}

This section is dedicated to the proof of Theorem 2.1 for the case of $T_{i}>0$. For this purpose, we need only to derive a uniform bound for the remainder equation (2.28). To slightly simplify the presentation, we assume that (2.28) has smooth solutions in a small time $\tau_{\varepsilon}$ dependent on $\varepsilon$. Let $\tilde{C}$ be a constant, which will be determined later, much larger than the bound of $\left\|\left(n_{R}^{\varepsilon}, \mathbf{u}_{R}^{\varepsilon}, \phi_{R}^{\varepsilon}\right)\right\|_{s^{\prime}}$, such that on $\left[0, \tau_{\varepsilon}\right]$

$$
\sup _{\left[0, \tau_{\varepsilon}\right]}\left\|\left(n_{R}^{\varepsilon}, \mathbf{u}_{R}^{\varepsilon}, \phi_{R}^{\varepsilon}\right)\right\|_{s^{\prime}} \leq \tilde{C} .
$$


We will prove that $\tau_{\varepsilon}>\tau_{0}$ as $\varepsilon \rightarrow 0$ for any $0<\tau_{0}<\tau_{*}$, where $\tau_{*}$ is the existence time of the limit equation (2.11) or (B.13). Recalling the expressions for $n$ and $\mathbf{u}$ in 2.27), we immediately know that there exists some $\varepsilon_{1}=\varepsilon_{1}(\tilde{C})>0$ such that on $\left[0, \tau_{\varepsilon}\right]$,

$$
1 / 2<n<3 / 2, \quad|\mathbf{u}| \leq 1 / 2,
$$

for all $0<\varepsilon<\varepsilon_{1}$.

Lemma 3.1. Let $\left(n_{R}^{\varepsilon}, \boldsymbol{u}_{R}^{\varepsilon}, \phi_{R}^{\varepsilon}\right)$ be a solution to (2.28) and $0 \leq k \leq s^{\prime} \leq s$ be an integer. There exist some $0<\varepsilon_{1}<1$ and $C, C_{0}>0$ such that for every $0<\varepsilon<\varepsilon_{1}$,

$$
\begin{gathered}
\left\|n_{R}^{\varepsilon}\right\|_{H^{k}}^{2} \leq C\left\|\phi_{R}^{\varepsilon}\right\|_{H^{k}}^{2}+C \varepsilon\left\|\bar{\nabla} \phi_{R}^{\varepsilon}\right\|_{H^{k}}^{2}+C \varepsilon^{2}\left\|\bar{\Delta} \phi_{R}^{\varepsilon}\right\|_{H^{k}}^{2}+C C_{0}^{2} \varepsilon^{2}, \\
\left\|\phi_{R}^{\varepsilon}\right\|_{H^{k}}^{2}+\varepsilon\left\|\bar{\nabla} \phi_{R}^{\varepsilon}\right\|_{H^{k}}^{2}+\varepsilon^{2}\left\|\bar{\Delta} \phi_{R}^{\varepsilon}\right\|_{H^{k}}^{2} \leq C\left\|n_{R}^{\varepsilon}\right\|_{H^{k}}^{2}+C C_{0}^{2} \varepsilon^{2} .
\end{gathered}
$$

Proof. Taking $H^{k}$ inner product of (2.28c) with $\phi_{R}^{\varepsilon}$, and integrating by parts, we have

$$
\begin{aligned}
\varepsilon\left\|\bar{\nabla} \phi_{R}^{\varepsilon}\right\|_{H^{k}}^{2}+\left\|\phi_{R}^{\varepsilon}\right\|_{H^{k}}^{2} & =\left\langle n_{R}^{\varepsilon}, \phi_{R}^{\varepsilon}\right\rangle_{H^{k}}-\left\langle\varepsilon \phi^{(1)} \phi_{R}^{\varepsilon}, \phi_{R}^{\varepsilon}\right\rangle_{H^{k}}-\left\langle\varepsilon^{3 / 2} R_{\phi}, \phi_{R}^{\varepsilon}\right\rangle_{H^{k}} \\
& \leq 2\left\|n_{R}^{\varepsilon}\right\|_{H^{k}}^{2}+\frac{1}{4}\left\|\phi_{R}^{\varepsilon}\right\|_{H^{k}}^{2}+C \varepsilon\left\|\phi_{R}^{\varepsilon}\right\|_{H^{k}}^{2}+2 \varepsilon^{3}\left\|R_{\phi}\right\|_{H^{k}}^{2} .
\end{aligned}
$$

From (3.1) and Lemma 2.1 there exist some constant $\varepsilon_{1}=\varepsilon_{1}(\tilde{C})>0$ and $C_{0}:=C_{1}(1)>0$, such that $C_{1}\left(\sqrt{\varepsilon}\left\|\phi_{R}^{\varepsilon}\right\|_{H^{\alpha}}\right) \leq C_{0}$ when $0<\varepsilon<\varepsilon_{1}$. This enables us to get $\left\|R_{\phi}\right\|_{H^{k}}^{2} \leq 2 C_{0}\left(1+\left\|\phi_{R}^{\varepsilon}\right\|_{H^{k}}^{2}\right)$. Therefore, there exists some $\varepsilon_{1}=\varepsilon_{1}(\tilde{C})>0$ (still denoted as $\varepsilon_{1}$ ) such that when $\varepsilon<\varepsilon_{1}$, we have

$$
C \varepsilon\left\|\phi_{R}^{\varepsilon}\right\|_{H^{k}}^{2}+2 \varepsilon^{3}\left\|R_{\phi}\right\|_{H^{k}}^{2} \leq \frac{1}{4}\left\|\phi_{R}^{\varepsilon}\right\|_{H^{k}}^{2}+4 C_{0}^{2} \varepsilon^{2} .
$$

Combining (3.5) and (3.6), we have

$$
\varepsilon\left\|\bar{\nabla} \phi_{R}^{\varepsilon}\right\|_{H^{k}}^{2}+\left\|\phi_{R}^{\varepsilon}\right\|_{H^{k}}^{2} \leq C\left\|n_{R}^{\varepsilon}\right\|_{H^{k}}^{2}+C C_{0}^{2} \varepsilon^{2}
$$

for some universal constant $C>0$.

Taking $H^{k}$ inner product of $\left(\overline{B .25 \mathrm{C}}\right.$ with $\varepsilon \bar{\Delta} \phi_{R}^{\varepsilon}$ and integrating by parts, we have similarly

$$
\begin{aligned}
& \varepsilon^{2}\left\|\bar{\Delta} \phi_{R}^{\varepsilon}\right\|_{H^{k}}^{2}+\varepsilon\left\|\bar{\nabla} \phi_{R}^{\varepsilon}\right\|_{H^{k}}^{2} \\
& \quad=\varepsilon\left\langle n_{R}^{\varepsilon}, \bar{\Delta} \phi_{R}^{\varepsilon}\right\rangle_{H^{k}}-\varepsilon^{2}\left\langle\phi^{(1)} \phi_{R}^{\varepsilon}, \bar{\Delta} \phi_{R}^{\varepsilon}\right\rangle_{H^{k}}+\varepsilon^{5 / 2}\left\langle\bar{\nabla} R_{\phi}, \bar{\nabla} \phi_{R}^{\varepsilon}\right\rangle_{H^{k}} \\
& \quad \leq 2\left\|n_{R}^{\varepsilon}\right\|_{H^{k}}^{2}+\frac{\varepsilon^{2}}{8}\left\|\bar{\Delta} \phi_{R}^{\varepsilon}\right\|_{H^{k}}^{2}+C \varepsilon^{2}\left\|\bar{\nabla} \phi_{R}^{\varepsilon}\right\|_{H^{k}}^{2}+\frac{\varepsilon}{8}\left\|\bar{\nabla} \phi_{R}^{\varepsilon}\right\|_{H^{k}}^{2}+2 \varepsilon^{4}\left\|\bar{\nabla} R_{\phi}\right\|_{H^{k}}^{2} .
\end{aligned}
$$

Similarly, from Lemma2.1, there exists some $\varepsilon_{1}=\varepsilon_{1}(\tilde{C})>0$ such that when $\varepsilon<\varepsilon_{1}$, we have

$$
\left\|\bar{\nabla} R_{\phi}\right\|_{H^{k}} \leq 2 C_{0}\left(1+\left\|\bar{\nabla} \phi_{R}^{\varepsilon}\right\|_{H^{k}}\right) .
$$

It then follows that

$$
\varepsilon^{2}\left\|\bar{\Delta} \phi_{R}^{\varepsilon}\right\|_{H^{k}}^{2}+\varepsilon\left\|\bar{\nabla} \phi_{R}^{\varepsilon}\right\|_{H^{k}}^{2} \leq C\left\|n_{R}^{\varepsilon}\right\|_{H^{k}}^{2}+C C_{0}^{2} \varepsilon^{2} .
$$

By adding (3.7) and (3.9) together, we know there exist some constants $\varepsilon_{1}>0, C$ and $C_{0}$ such that

$$
\varepsilon^{2}\left\|\bar{\Delta} \phi_{R}^{\varepsilon}\right\|_{H^{k}}^{2}+\varepsilon\left\|\bar{\nabla} \phi_{R}^{\varepsilon}\right\|_{H^{k}}^{2}+\left\|\phi_{R}^{\varepsilon}\right\|_{H^{k}}^{2} \leq C\left\|n_{R}^{\varepsilon}\right\|_{H^{k}}^{2}+C C_{0}^{2} \varepsilon^{2} .
$$

On the other hand, by taking $H^{\alpha}$ norm of $(\mathrm{B} .25 \mathrm{c})$, we have

$$
\begin{aligned}
\left\|n_{R}^{\varepsilon}\right\|_{H^{k}}^{2} & \leq \varepsilon^{2}\left\|\bar{\Delta} \phi_{R}^{\varepsilon}\right\|_{H^{k}}^{2}+C\left\|\phi_{R}^{\varepsilon}\right\|_{H^{k}}^{2}+C \varepsilon^{3}\left\|R_{\phi}\right\|_{H^{k}}^{2} \\
& \leq \varepsilon^{2}\left\|\bar{\Delta} \phi_{R}^{\varepsilon}\right\|_{H^{k}}^{2}+C\left\|\phi_{R}^{\varepsilon}\right\|_{H^{k}}^{2}+C C_{0}^{2} \varepsilon^{2} .
\end{aligned}
$$

Combining (3.10) and (3.11), we complete the proof. 
Lemma 3.2. Let $\left(n_{R}^{\varepsilon}, \boldsymbol{u}_{R}^{\varepsilon}, \phi_{R}^{\varepsilon}\right)$ be a solution to $\left(\mathbb{B . 2 5}\right.$ and $s^{\prime} \leq s$ be an integer. Then for any $1 \leq k \leq s^{\prime}$, there exist some $0<\varepsilon_{1}<1$ and $C>0$ such that for every $0<\varepsilon<\varepsilon_{1}$,

$$
\left\|\varepsilon \partial_{t} n_{R}^{\varepsilon}\right\|_{H^{k-1}}^{2} \leq C\left\{1+\left\|\boldsymbol{u}_{R}^{\varepsilon}\right\|_{H^{\delta+1}}^{2}+\left\|n_{R}^{\varepsilon}\right\|_{H^{\delta+1}}^{2}\right\}
$$

or equivalently,

$$
\left\|\varepsilon \partial_{t} n_{R}^{\varepsilon}\right\|_{H^{k-1}}^{2} \leq C\left\{1+\left\|\boldsymbol{u}_{R}^{\varepsilon}\right\|_{H^{\delta+1}}^{2}+\left\|\phi_{R}^{\varepsilon}\right\|_{\delta+1}^{2}\right\}
$$

where $\delta=\max \{2, k-1\}$.

Proof. Multiply (2.28a) by $\varepsilon$, and then take $H^{k-1}$ norm to obtain

$$
\begin{aligned}
\left\|\varepsilon \partial_{t} n_{R}^{\varepsilon}\right\|_{H^{k-1}}^{2} \leq & C\left\|\bar{\nabla} n_{R}^{\varepsilon}\right\|_{H^{k-1}}^{2}+C\left\|\mathbf{u} \cdot \bar{\nabla} n_{R}^{\varepsilon}\right\|_{H^{k-1}}^{2}+C\left\|n \bar{\nabla} \cdot \mathbf{u}_{R}^{\varepsilon}\right\|_{H^{k-1}}^{2} \\
& +C \varepsilon^{2}\left\|n_{R}^{\varepsilon} \cdot \bar{\nabla} \widetilde{\mathbf{u}}\right\|_{H^{k-1}}^{2}+C \varepsilon^{2}\left\|\mathbf{u}_{R}^{\varepsilon} \cdot \bar{\nabla} \widetilde{n}\right\|_{H^{k-1}}^{2}+C \varepsilon^{4}\left\|R_{n}\right\|_{H^{k-1}}^{2} .
\end{aligned}
$$

Recall that $n=1+\varepsilon \widetilde{n}+\varepsilon^{2} n_{R}^{\varepsilon}$ and $\mathbf{u}=\varepsilon \widetilde{\mathbf{u}}+\varepsilon^{2} \mathbf{u}_{R}^{\varepsilon}$ in 2.27). By Lemma C.1 we have

$$
\begin{aligned}
\left\|\mathbf{u} \cdot \bar{\nabla} n_{R}^{\varepsilon}\right\|_{H^{k-1}}^{2} & \leq C \varepsilon^{2}\left\|\left(\widetilde{\mathbf{u}}+\varepsilon \mathbf{u}_{R}^{\varepsilon}\right) \cdot \bar{\nabla} n_{R}^{\varepsilon}\right\|_{H^{k-1}}^{2} \\
& \leq C \varepsilon^{2}\left\|\bar{\nabla} n_{R}^{\varepsilon}\right\|_{H^{k-1}}^{2}+C \varepsilon^{4}\left\{\left\|\mathbf{u}_{R}^{\varepsilon}\right\|_{H^{k-1}}^{2}\left\|\bar{\nabla} n_{R}^{\varepsilon}\right\|_{L^{\infty}}^{2}+\left\|\bar{\nabla} n_{R}^{\varepsilon}\right\|_{H^{k-1}}^{2}\left\|\mathbf{u}_{R}^{\varepsilon}\right\|_{L^{\infty}}^{2}\right\} \\
& \leq C \varepsilon^{2}\left\{1+C\left(\varepsilon^{2}\left\|\mathbf{u}_{R}^{\varepsilon}\right\|_{H^{\delta}}^{2}\right)\right\}\left\|\bar{\nabla} n_{R}^{\varepsilon}\right\|_{H^{\delta}}^{2},
\end{aligned}
$$

where $\delta=\max \{2, k-1\}$. Similarly, we have

$$
\begin{aligned}
\left\|n \bar{\nabla} \cdot \mathbf{u}_{R}^{\varepsilon}\right\|_{H^{k-1}}^{2} & \leq\left\|\left(1+\varepsilon \widetilde{n}+\varepsilon^{2} n_{R}^{\varepsilon}\right) \bar{\nabla} \cdot \mathbf{u}_{R}^{\varepsilon}\right\|_{H^{k-1}}^{2} \\
& \leq C\left\|\bar{\nabla} \mathbf{u}_{R}^{\varepsilon}\right\|_{H^{k-1}}^{2}+C \varepsilon^{4}\left\{\left\|\bar{\nabla} \mathbf{u}_{R}^{\varepsilon}\right\|_{H^{k-1}}^{2}\left\|n_{R}^{\varepsilon}\right\|_{L^{\infty}}^{2}+\left\|n_{R}^{\varepsilon}\right\|_{H^{k-1}}^{2}\left\|\bar{\nabla} \mathbf{u}_{R}^{\varepsilon}\right\|_{L^{\infty}}^{2}\right\} \\
& \leq C\left\{1+\varepsilon^{2}\left\|\bar{\nabla} \mathbf{u}_{R}^{\varepsilon}\right\|_{H^{\delta}}^{2}\right\}\left\{\left\|n_{R}^{\varepsilon}\right\|_{H^{\delta}}^{2}+\left\|\mathbf{u}_{R}^{\varepsilon}\right\|_{H^{\delta+1}}^{2}\right\},
\end{aligned}
$$

where $\delta=\max \{2, k-1\}$. On the other hand, since $\widetilde{\mathbf{u}}, \widetilde{n} \in H^{s}$ and $R_{n}$ depends only on $\widetilde{\mathbf{u}}$ and $\widetilde{n}$, the last three terms on the RHS of (3.14) are easily bounded by

$$
C \varepsilon^{2}\left\{1+\left\|\mathbf{u}_{R}^{\varepsilon}\right\|_{H^{k-1}}^{2}+\left\|n_{R}^{\varepsilon}\right\|_{H^{k-1}}^{2}\right\}
$$

Inserting (3.15)-3.17) into 3.14, we obtain

$$
\left\|\varepsilon \partial_{t} n_{R}^{\varepsilon}\right\|_{H^{k-1}}^{2} \leq C\left\{1+\varepsilon^{2}\left\|\mathbf{u}_{R}^{\varepsilon}\right\|_{H^{\delta+1}}^{2}\right\}\left\{1+\left\|n_{R}^{\varepsilon}\right\|_{H^{\delta+1}}^{2}+\left\|\mathbf{u}_{R}^{\varepsilon}\right\|_{H^{\delta}}^{2}\right\},
$$

where $\delta=\max \{2, k-1\}$. Since $\left\|\mathbf{u}_{R}^{\varepsilon}\right\|_{H^{\delta+1}}^{2} \leq\left\|\left(u_{R}^{\varepsilon}, \phi_{R}^{\varepsilon}\right)\right\|^{2} \leq \tilde{C}$ by assumption (3.1), there exists some $\varepsilon_{1} \in(0,1)$ depending on $\tilde{C}$ such that $\varepsilon^{2}\left\|\mathbf{u}_{R}^{\varepsilon}\right\|_{H^{\delta+1}}^{2} \leq 1$ when $0<\varepsilon<\varepsilon_{1}$. Therefore (3.12) is proved. Invoking Lemma 3.1 we obtain

$$
\left\|\varepsilon \partial_{t} n_{R}^{\varepsilon}\right\|_{H^{k-1}}^{2} \leq C\left\{1+\left\|\mathbf{u}_{R}^{\varepsilon}\right\|_{H^{\delta+1}}^{2}+\left\|\phi_{R}^{\varepsilon}\right\|_{\delta+1}^{2}\right\}
$$

for any $1 \leq k \leq s^{\prime}$, where $\delta=\max \{2, k-1\}$ and $\left\|\phi_{R}^{\varepsilon}\right\|_{\delta+1}^{2}$ is given by (2.31). The proof is complete.

Lemma 3.3. Let $\left(n_{R}^{\varepsilon}, \boldsymbol{u}_{R}^{\varepsilon}, \phi_{R}^{\varepsilon}\right)$ be a solution to (B.25) and $s^{\prime} \leq s$ be an integer. Then for any $1 \leq k \leq s^{\prime}$, there exist some $0<\varepsilon_{1}<1$ and $C, C_{0}>0$ such that for every $0<\varepsilon<\varepsilon_{1}$,

$$
\left\|\partial_{t} \phi_{R}^{\varepsilon}\right\|_{k-1}^{2} \leq C\left\|\partial_{t} n_{R}^{\varepsilon}\right\|_{H^{k-1}}^{2}+C \varepsilon\left\|\phi_{R}^{\varepsilon}\right\|_{H^{k-1}}^{2}+C C_{0}^{2} \varepsilon .
$$

where $\delta=\max \{2, k-1\}$. 
Proof. Let $1 \leq k \leq s^{\prime}$. We take $\partial_{t}$ of (2.28c) and then take $H^{k-1}$ inner product with $\partial_{t} \phi_{R}^{\varepsilon}$ to obtain

$$
\begin{aligned}
\varepsilon \| \bar{\nabla} & \partial_{t} \phi_{R}^{\varepsilon}\left\|_{H^{k-1}}^{2}+\right\| \partial_{t} \phi_{R}^{\varepsilon} \|_{H^{k-1}}^{2} \\
& =\left\langle\partial_{t} n_{R}^{\varepsilon}, \partial_{t} \phi_{R}^{\varepsilon}\right\rangle_{H^{k-1}}-\varepsilon\left\langle\partial_{t}\left(\phi^{(1)} \phi_{R}^{\varepsilon}\right), \partial_{t} \phi_{R}^{\varepsilon}\right\rangle_{H^{k-1}}-\varepsilon^{3 / 2}\left\langle\partial_{t} R_{\phi}, \partial_{t} \phi_{R}^{\varepsilon}\right\rangle_{H^{k-1}} \\
& \leq 2\left\|\partial_{t} n_{R}^{\varepsilon}\right\|_{H^{k-1}}^{2}+\frac{1}{4}\left\|\partial_{t} \phi_{R}^{\varepsilon}\right\|_{H^{k-1}}^{2}+C \varepsilon\left\|\partial_{t} \phi_{R}^{\varepsilon}\right\|_{H^{k-1}}^{2}+C \varepsilon\left\|\phi_{R}^{\varepsilon}\right\|_{H^{k-1}}^{2}+2 \varepsilon^{3}\left\|\partial_{t} R_{\phi}\right\|_{H^{k-1}}^{2} .
\end{aligned}
$$

The fourth term in the last line comes from the term $\partial_{t}\left(\phi^{(1)} \phi_{R}^{\varepsilon}\right)$ when $\partial_{t}$ acts on $\phi^{(1)}$. As in the proof of Lemma 3.1, by (2.23) in Lemma2.1, there exists some $\varepsilon_{1}=\varepsilon_{1}(\tilde{C})>0$ such that when $\varepsilon<\varepsilon_{1}$, we have

$$
\varepsilon\left\|\bar{\nabla} \partial_{t} \phi_{R}^{\varepsilon}\right\|_{H^{k-1}}^{2}+\left\|\partial_{t} \phi_{R}^{\varepsilon}\right\|_{H^{k-1}}^{2} \leq C\left\|\partial_{t} n_{R}^{\varepsilon}\right\|_{H^{k-1}}^{2}+C \varepsilon\left\|\phi_{R}^{\varepsilon}\right\|_{H^{k-1}}^{2}+C C_{0}^{2} \varepsilon^{2}
$$

for some universal constant $C>0$.

On the other hand, by taking $\partial_{t}$ of (B.25c) and then taking $H^{k-1}$ inner product with $\varepsilon \partial_{t} \bar{\Delta} \phi_{R}^{\varepsilon}$, we obtain

$$
\varepsilon^{2}\left\|\partial_{t} \bar{\Delta} \phi_{R}^{\varepsilon}\right\|_{H^{k-1}}^{2}+\varepsilon\left\|\partial_{t} \bar{\nabla} \phi_{R}^{\varepsilon}\right\|_{H^{k-1}}^{2} \leq C\left\|\partial_{t} n_{R}^{\varepsilon}\right\|_{H^{k-1}}^{2}+C \varepsilon^{2}\left(\left\|\partial_{t} \phi_{R}^{\varepsilon}\right\|_{H^{k-1}}^{2}+\left\|\phi_{R}^{\varepsilon}\right\|_{H^{k-1}}^{2}\right)+C C_{0}^{2} \varepsilon,
$$

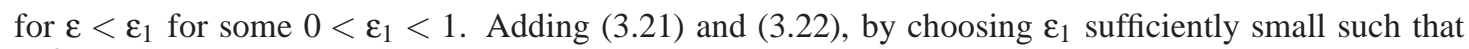
$C \varepsilon_{1}^{2} \leq 1 / 2$, we obtain

$$
\varepsilon^{2}\left\|\partial_{t} \bar{\Delta} \phi_{R}^{\varepsilon}\right\|_{H^{k-1}}^{2}+\varepsilon\left\|\partial_{t} \bar{\nabla} \phi_{R}^{\varepsilon}\right\|_{H^{k-1}}^{2}+\left\|\partial_{t} \phi_{R}^{\varepsilon}\right\|_{H^{k-1}}^{2} \leq C\left\|\partial_{t} n_{R}^{\varepsilon}\right\|_{H^{k-1}}^{2}+C \varepsilon\left\|\phi_{R}^{\varepsilon}\right\|_{H^{k-1}}^{2}+C C_{0}^{2} \varepsilon .
$$

The proof is complete.

Corollary 3.1. Under the same assumptions of Lemma 3.3 we have

$$
\left\|\varepsilon \partial_{t} \phi_{R}^{\varepsilon}\right\|_{k-1}^{2} \leq C\left\{1+\left\|\left(\boldsymbol{u}_{R}^{\varepsilon}, n_{R}^{\varepsilon}, \phi_{R}^{\varepsilon}\right)\right\|_{H^{\delta+1}}^{2}\right\},
$$

or equivalently,

$$
\left\|\mid \varepsilon \partial_{t} \phi_{R}^{\varepsilon}\right\|_{k-1}^{2} \leq C\left\{1+\left\|u_{R}^{\varepsilon}\right\|_{H^{\delta+1}}^{2}+\left\|\phi_{R}^{\varepsilon}\right\|_{\delta+1}^{2}\right\}
$$

where $\delta=\max \{2, k-1\}$.

Proof. The proof is complete by multiplying (3.20) with $\varepsilon^{2}$ and then using Lemma 3.2

Corollary 3.2. Let $\alpha$ be a multi-index with $|\alpha|=k$, then

$$
\begin{cases}\varepsilon^{3}\left\|\varepsilon \partial_{t} \bar{\nabla} \partial_{x}^{\alpha} \phi_{R}^{\varepsilon}\right\|_{L^{2}}^{2} \leq C \varepsilon^{2}\left\|\varepsilon \partial_{t} \bar{\Delta} \phi_{R}^{\varepsilon}\right\|_{H^{k-1}}^{2}, & d=2 ; \\ \varepsilon^{2}\left\|\varepsilon \partial_{t} \bar{\nabla} \partial_{x}^{\alpha} \phi_{R}^{\varepsilon}\right\|_{L^{2}}^{2} \leq C \varepsilon^{2}\left\|\varepsilon \partial_{t} \bar{\Delta} \phi_{R}^{\varepsilon}\right\|_{H^{k-1}}^{2}, & d=3,\end{cases}
$$

where $x \in \mathbb{R}^{d}$ and $\bar{\nabla}$ is defined in (2.24) and $\bar{\Delta}=\bar{\nabla} \cdot \bar{\nabla}$.

Proof. By Reisz theorem [22], we have

$$
\left\|\sqrt{\varepsilon} \partial_{x_{i}} \bar{\nabla} f\right\|_{L^{2}} \leq C\|\bar{\Delta} f\|_{L^{2}}
$$

for those $f$ that makes sense. The proof is complete by letting $f=\varepsilon^{2} \partial_{t} \phi_{R}^{\varepsilon}$.

Proposition 3.1. Let $s^{\prime} \geq 4$ be an integer and $\left(n_{R}^{\varepsilon}, \boldsymbol{u}_{R}^{\varepsilon}, \phi_{R}^{\varepsilon}\right)$ be a solution to 2.28). Then for any integer $0 \leq k \leq s^{\prime}$, there holds

$$
\begin{aligned}
\frac{1}{2} \frac{d}{d t}\left\|\partial_{x}^{\alpha} \boldsymbol{u}_{R}^{\varepsilon}\right\|_{L^{2}}^{2} & +\frac{1}{2} \frac{d}{d t} \int \frac{1+\varepsilon \phi^{(1)}+\varepsilon^{2} \phi_{R}^{\varepsilon}}{n}\left|\partial_{x}^{\alpha} \phi_{R}^{\varepsilon}\right|^{2} \\
& +\frac{1}{2} \frac{d}{d t} \int \frac{\varepsilon}{n}\left|\bar{\nabla} \partial_{x}^{\alpha} \phi_{R}^{\varepsilon}\right|^{2}+\frac{1}{2} \frac{d}{d t} \int \frac{T_{i}}{n^{2}}\left|\partial_{x}^{\alpha} n_{R}^{\varepsilon}\right|^{2} d x \\
\leq & C C_{1}\left(C_{1}+\varepsilon\left\|\left(\boldsymbol{u}_{R}^{\varepsilon}, n_{R}^{\varepsilon}, \phi_{R}^{\varepsilon}\right)\right\|_{H^{s^{\prime}}}^{2}\left\{1+\left\|\left(\boldsymbol{u}_{R}^{\varepsilon}, n_{R}^{\varepsilon}, \phi_{R}^{\varepsilon}\right)\right\|_{H^{s^{\prime}}}^{2}\right\}\right.
\end{aligned}
$$

where $\alpha$ is any multi-index with $|\alpha|=k$. 
For clarity, we divide the proof of this proposition into the following four lemmas.

Lemma 3.4. Let $s^{\prime} \geq 4, k \leq s^{\prime}$ be two non-negative integers and $\alpha$ be any multi-index with $|\alpha|=k$. Then for any solution $\left(n_{R}^{\varepsilon}, \boldsymbol{u}_{R}^{\varepsilon}, \phi_{R}^{\varepsilon}\right)$ of $(2.28)$, we have

$$
\begin{aligned}
\frac{1}{2} \frac{d}{d t}\left\|\partial_{x}^{\alpha} \boldsymbol{u}_{R}^{\varepsilon}\right\|_{L^{2}}^{2}+\frac{1}{2} \frac{d}{d t} \int \frac{1+\varepsilon \phi^{(1)}+\varepsilon^{2} \phi_{R}^{\varepsilon}}{n}\left|\partial_{x}^{\alpha} \phi_{R}^{\varepsilon}\right|^{2}+\frac{1}{2} \frac{d}{d t} \int \frac{\varepsilon}{n}\left|\bar{\nabla} \partial_{x}^{\alpha} \phi_{R}^{\varepsilon}\right|^{2} \\
\leq C C_{1}\left(C_{1}+\varepsilon\left\|\left(\boldsymbol{u}_{R}^{\varepsilon}, n_{R}^{\varepsilon}, \phi_{R}^{\varepsilon}\right)\right\|_{H^{s^{\prime}}}^{2}\right)\left\{1+\left\|\left(\boldsymbol{u}_{R}^{\varepsilon}, n_{R}^{\varepsilon}, \phi_{R}^{\varepsilon}\right)\right\|_{H^{s^{\prime}}}^{2}\right\}+I_{71},
\end{aligned}
$$

where

$$
I_{71}=-T_{i}\left\langle\frac{1}{\varepsilon n} \partial_{x}^{\alpha} \bar{\nabla} n_{R}^{\varepsilon}, \partial_{x}^{\alpha} \boldsymbol{u}_{R}^{\varepsilon}\right\rangle_{0}
$$

Proof. Let $\alpha$ be any multi-index with $|\alpha|=k$. We take $\partial_{x}^{\alpha}$ of $2.28 \mathrm{~b}$ ) and then take $L^{2}$ inner product with $\partial_{x}^{\alpha} \mathbf{u}_{R}^{\varepsilon}$, to obtain

$$
\begin{aligned}
\frac{1}{2} \frac{d}{d t}\left\|\partial_{x}^{\alpha} \mathbf{u}_{R}^{\varepsilon}\right\|_{L^{2}}^{2}= & \frac{V}{\varepsilon}\left\langle\partial_{x_{1}} \partial_{x}^{\alpha} \mathbf{u}_{R}^{\varepsilon}, \partial_{x}^{\alpha} \mathbf{u}_{R}^{\varepsilon}\right\rangle_{0}-\frac{1}{\varepsilon}\left\langle\partial_{x}^{\alpha}\left(\mathbf{u} \cdot \bar{\nabla} \mathbf{u}_{R}^{\varepsilon}\right), \partial_{x}^{\alpha} \mathbf{u}_{R}^{\varepsilon}\right\rangle_{0}-\left\langle\partial_{x}^{\alpha}\left(\mathbf{u}_{R}^{\varepsilon} \cdot \bar{\nabla} \widetilde{\mathbf{u}}\right), \partial_{x}^{\alpha} \mathbf{u}_{R}^{\varepsilon}\right\rangle_{0} \\
& -\varepsilon\left\langle\partial_{x}^{\alpha} \mathbf{R}_{\mathbf{u}}, \partial_{x}^{\alpha} \mathbf{u}_{R}^{\varepsilon}\right\rangle_{0}+\frac{b}{\varepsilon^{3 / 2}}\left\langle\partial_{x}^{\alpha} \mathbf{u}_{R}^{\varepsilon} \times \mathbf{e}_{1}, \partial_{x}^{\alpha} \mathbf{u}_{R}^{\varepsilon}\right\rangle_{0}-\frac{1}{\varepsilon}\left\langle\partial_{x}^{\alpha} \bar{\nabla} \phi_{R}^{\varepsilon}, \partial_{x}^{\alpha} \mathbf{u}_{R}^{\varepsilon}\right\rangle_{0} \\
& -T_{i}\left\langle\partial_{x}^{\alpha}\left(\frac{\bar{\nabla} n_{R}^{\varepsilon}}{\varepsilon n}\right), \partial_{x}^{\alpha} \mathbf{u}_{R}^{\varepsilon}\right\rangle_{0}+T_{i}\left\langle\partial_{x}^{\alpha}\left(\frac{\mathbf{p}}{n} n_{R}^{\varepsilon}\right), \partial_{x}^{\alpha} \mathbf{u}_{R}^{\varepsilon}\right\rangle_{0}+T_{i}\left\langle\partial_{x}^{\alpha}\left(\frac{\varepsilon \mathbf{R}_{T}}{n}\right), \partial_{x}^{\alpha} \mathbf{u}_{R}^{\varepsilon}\right\rangle_{0} \\
= & : I_{1}+\cdots+I_{9} .
\end{aligned}
$$

Estimate of $I_{1}$. By integrating by parts, we have $I_{1}=0$.

Estimate of $I_{2}$. Using the commutator, we have

$$
I_{2}=-\frac{1}{\varepsilon}\left\langle\mathbf{u} \cdot \bar{\nabla} \partial_{x}^{\alpha} \mathbf{u}_{R}^{\varepsilon}, \partial_{x}^{\alpha} \mathbf{u}_{R}^{\varepsilon}\right\rangle_{0}-\frac{1}{\varepsilon}\left\langle\left[\partial_{x}^{\alpha}, \mathbf{u}\right] \cdot \bar{\nabla} \mathbf{u}_{R}^{\varepsilon}, \partial_{x}^{\alpha} \mathbf{u}_{R}^{\varepsilon}\right\rangle_{0}=I_{21}+I_{22}
$$

Since

$$
\begin{aligned}
-\left\langle\mathbf{u} \cdot \bar{\nabla} \partial_{x}^{\alpha} \mathbf{u}_{R}^{\varepsilon}, \partial_{x}^{\alpha} \mathbf{u}_{R}^{\varepsilon}\right\rangle_{0} & =\left\langle\bar{\nabla} \cdot \mathbf{u} \mathbf{u}_{R}^{\varepsilon}, \mathbf{u}_{R}^{\varepsilon}\right\rangle_{0}+\left\langle\mathbf{u} \otimes \partial_{x}^{\alpha} \mathbf{u}_{R}^{\varepsilon}, \bar{\nabla} \partial_{x}^{\alpha} \mathbf{u}_{R}^{\varepsilon}\right\rangle_{0} \\
& =\left\langle\bar{\nabla} \cdot \mathbf{u} \partial_{x}^{\alpha} \mathbf{u}_{R}^{\varepsilon}, \partial_{x}^{\alpha} \mathbf{u}_{R}^{\varepsilon}\right\rangle_{0}+\left\langle\mathbf{u} \cdot \bar{\nabla} \partial_{x}^{\alpha} \mathbf{u}_{R}^{\varepsilon}, \partial_{x}^{\alpha} \mathbf{u}_{R}^{\varepsilon}\right\rangle_{0}
\end{aligned}
$$

we have

$$
-\left\langle\mathbf{u} \cdot \bar{\nabla} \partial_{x}^{\alpha} \mathbf{u}_{R}^{\varepsilon}, \partial_{x}^{\alpha} \mathbf{u}_{R}^{\varepsilon}\right\rangle_{0}=\frac{1}{2}\left\langle\bar{\nabla} \cdot \mathbf{u} \partial_{x}^{\alpha} \mathbf{u}_{R}^{\varepsilon}, \partial_{x}^{\alpha} \mathbf{u}_{R}^{\varepsilon}\right\rangle_{0}
$$

Recalling 2.27], we have by Sobolev embedding,

$$
\begin{aligned}
\left|I_{21}\right| & \leq C\left\|\bar{\nabla}\left(\widetilde{\mathbf{u}}+\varepsilon \mathbf{u}_{R}^{\varepsilon}\right)\right\|_{L^{\infty}}\left\|\partial_{x}^{\alpha} \mathbf{u}_{R}^{\varepsilon}\right\|_{L^{2}}^{2} \\
& \leq C\left(1+\varepsilon\left\|\mathbf{u}_{R}^{\varepsilon}\right\|_{H^{3}}\right)\left\|\partial_{x}^{\alpha} \mathbf{u}_{R}^{\varepsilon}\right\|_{L^{2}}^{2} .
\end{aligned}
$$

On the other hand, recalling [2.27), by commutator estimates in [14], we have

$$
\begin{aligned}
\left\|\left[\partial_{x}^{\alpha}, \mathbf{u}\right] \cdot \bar{\nabla} \mathbf{u}_{R}^{\varepsilon}\right\|_{L^{2}} & \leq C\left(\left\|\bar{\nabla} \mathbf{u}_{R}^{\varepsilon}\right\|_{H^{k-1}}\|\nabla \mathbf{u}\|\left\|_{L^{\infty}}+\right\| \mathbf{u}\left\|_{\dot{H}^{k}}\right\| \bar{\nabla} \mathbf{u}_{R}^{\varepsilon} \|_{L^{\infty}}\right) \\
& \leq C \varepsilon\left(\left\|\mathbf{u}_{R}^{\varepsilon}\right\|_{\dot{H}^{k}}\left(1+\varepsilon\left\|\nabla \mathbf{u}_{R}^{\varepsilon}\right\|_{L^{\infty}}\right)+\left\|\nabla \mathbf{u}_{R}^{\varepsilon}\right\|_{L^{\infty}}\left(1+\varepsilon\left\|\mathbf{u}_{R}^{\varepsilon}\right\|_{H^{k}}\right)\right) \\
& \leq C \varepsilon\left(1+\varepsilon\left\|\mathbf{u}_{R}^{\varepsilon}\right\|_{H^{s^{\prime}}}\right)\left\|\mathbf{u}_{R}^{\varepsilon}\right\|_{H^{s^{\prime}}},
\end{aligned}
$$

where $k \leq s^{\prime}$ and $s^{\prime} \geq 3$. For $I_{22}$ in 3.30 , we then have

$$
\left|I_{22}\right| \leq C\left(1+\left\|\mathbf{u}_{R}^{\varepsilon}\right\|_{H^{s^{\prime}}}\right)\left\|\mathbf{u}_{R}^{\varepsilon}\right\|_{H^{s^{\prime}}}^{2}
$$


Combining (3.31) and (3.32), we have

$$
\left|I_{2}\right| \leq C\left(1+\left\|\mathbf{u}_{R}^{\varepsilon}\right\|_{H^{s^{\prime}}}\right)\left\|\mathbf{u}_{R}^{\varepsilon}\right\|_{H^{s^{\prime}}}^{2} .
$$

Estimate of $I_{3}$. By multiplicative estimates in [14] and (2.30), we have

$$
\begin{aligned}
\left\|\partial_{x}^{\alpha}\left(\mathbf{u}_{R}^{\varepsilon} \cdot \bar{\nabla} \widetilde{\mathbf{u}}\right)\right\|_{L^{2}} & \leq C\left(\left\|\mathbf{u}_{R}^{\varepsilon}\right\|_{\dot{H}^{k}}\|\bar{\nabla} \widetilde{\mathbf{u}}\|_{L^{\infty}}+\left\|\mathbf{u}_{R}^{\varepsilon}\right\|_{L^{\infty}}\|\bar{\nabla} \widetilde{\mathbf{u}}\|_{\dot{H}^{k}}\right) \\
& \leq C\left\|\mathbf{u}_{R}^{\varepsilon}\right\|_{H^{s^{\prime}}},
\end{aligned}
$$

which follows that

$$
\left|I_{3}\right| \leq C\left\|\mathbf{u}_{R}^{\varepsilon}\right\|_{H^{s^{\prime}}}^{2}
$$

Estimate of $I_{4}$. Similarly, from (2.30, we have

$$
\left|I_{4}\right| \leq C\left\|\mathbf{u}_{R}^{\varepsilon}\right\|_{H^{k}}^{2}+C \varepsilon^{2} .
$$

Estimate of $I_{5}$. It is easy to see $I_{5}=0$.

Estimate of $I_{7}$. Recalling $I_{7}$ in 3.29), we have

$$
\begin{aligned}
I_{7} & =-T_{i}\left\langle\partial_{x}^{\alpha}\left(\frac{\bar{\nabla} n_{R}^{\varepsilon}}{\varepsilon n}\right), \partial_{x}^{\alpha} \mathbf{u}_{R}^{\varepsilon}\right\rangle_{0} \\
& =-T_{i}\left\langle\frac{1}{\varepsilon n} \partial_{x}^{\alpha} \bar{\nabla} n_{R}^{\varepsilon}, \partial_{x}^{\alpha} \mathbf{u}_{R}^{\varepsilon}\right\rangle_{0}-T_{i}\left\langle\left[\partial^{\alpha}, \frac{1}{\varepsilon n}\right] \bar{\nabla} n_{R}^{\varepsilon}, \partial_{x}^{\alpha} \mathbf{u}_{R}^{\varepsilon}\right\rangle_{0} \\
& =: I_{71}+I_{72} .
\end{aligned}
$$

We note that when $k=0$, there is no such commutator term. From $\underline{3.2}$, we have

$$
\begin{aligned}
\left\|\nabla\left(\frac{1}{\varepsilon n}\right)\right\|_{L^{\infty}} & \leq C\|\nabla \widetilde{n}\|_{L^{\infty}}+C \varepsilon\left\|\nabla n_{R}^{\varepsilon}\right\|_{L^{\infty}} \\
& \leq C+C \varepsilon\left\|n_{R}^{\varepsilon}\right\|_{H^{3}},
\end{aligned}
$$

where we have used the expression (2.27). Using 2.27, 3.1) and (3.2), we have

$$
\left\|\frac{1}{\varepsilon n}\right\|_{\dot{H}^{k}} \leq C+\varepsilon C_{1}\left\|n_{R}^{\varepsilon}\right\|_{H^{k}}
$$

for $k \geq 1$, where $C_{1}=C_{1}(\varepsilon \tilde{C})$ is some constant depending on $\varepsilon \tilde{C}$. Since $s^{\prime} \geq 3$ and $k \leq s^{\prime}$, we have

$$
\begin{aligned}
\left\|\left[\partial_{x}^{\alpha}, \frac{1}{\varepsilon n}\right] \bar{\nabla} n_{R}^{\varepsilon}\right\|_{L^{2}} & \leq C\left(\left\|\bar{\nabla} n_{R}^{\varepsilon}\right\|_{\dot{H}^{k-1}}\left\|\nabla\left(\frac{1}{\varepsilon n}\right)\right\|_{L^{\infty}}+\left\|\bar{\nabla} n_{R}^{\varepsilon}\right\|_{L^{\infty}}\left\|\frac{1}{\varepsilon n}\right\|_{\dot{H}^{k}}\right) \\
& \leq C\left(1+\varepsilon C_{1}\left\|n_{R}^{\varepsilon}\right\|_{H^{s^{\prime}}}\right)\left\|n_{R}^{\varepsilon}\right\|_{H^{s^{\prime}}},
\end{aligned}
$$

where we have used (3.35) and (3.36). Therefore, we have

$$
\left|I_{72}\right| \leq C\left(1+\varepsilon C_{1}\left\|n_{R}^{\varepsilon}\right\|_{H^{s^{\prime}}}\right)\left(\left\|n_{R}^{\varepsilon}\right\|_{H^{s^{\prime}}}^{2}+\left\|\mathbf{u}_{R}^{\varepsilon}\right\|_{H^{s^{\prime}}}^{2}\right) .
$$

Estimate of $I_{8}$. Recall

$$
I_{8}=T_{i} \sum_{|\alpha|=k}\left\langle\partial_{x}^{\alpha}\left(\frac{\mathbf{p}}{n} n_{R}^{\varepsilon}\right), \partial_{x}^{\alpha} \mathbf{u}_{R}^{\varepsilon}\right\rangle_{0}
$$

From (3.1) and (3.2), we have

$$
\left\|\partial_{x}^{\alpha}\left(\frac{\mathbf{p}}{n}\right)\right\| \leq C \varepsilon+C C_{1} \varepsilon^{2}\left\|n_{R}^{\varepsilon}\right\|_{H^{k}}
$$


where $C_{1}=C_{1}(\varepsilon \tilde{C})$ is a constant depending on $\varepsilon \tilde{C}$. By multiplicative estimate, we then have

$$
\begin{aligned}
\left\|\partial_{x}^{\alpha}\left(\frac{\mathbf{p}}{n} n_{R}^{\varepsilon}\right)\right\| & \leq C\left(\left\|\frac{\mathbf{p}}{n}\right\|_{L^{\infty}}\left\|n_{R}^{\varepsilon}\right\|_{\dot{H}^{k}}+\left\|n_{R}^{\varepsilon}\right\|_{L^{\infty}}\left\|\frac{\mathbf{p}}{n}\right\|_{\dot{H}^{k}}\right) \\
& \leq C\left\|n_{R}^{\varepsilon}\right\|_{\dot{H}^{k}}+C \varepsilon\left\|n_{R}^{\varepsilon}\right\|_{H^{3}}+C C_{1} \varepsilon^{2}\left\|n_{R}^{\varepsilon}\right\|_{H^{3}}\left\|n_{R}^{\varepsilon}\right\|_{H^{k}} \\
& \leq C\left(1+\varepsilon C_{1}\right)\left\|n_{R}^{\varepsilon}\right\|_{H^{k}}+C \varepsilon\left\|n_{R}^{\varepsilon}\right\|_{H^{3}},
\end{aligned}
$$

where we have used $\left\|n_{R}^{\varepsilon}\right\|_{H^{3}} \leq \tilde{C}$ by (3.1) and $s^{\prime} \geq 3$. Therefore, from (3.37), we have

$$
\begin{aligned}
\left|I_{8}\right| & \leq C\left(1+\varepsilon C_{1}\right)\left\|n_{R}^{\varepsilon}\right\|_{H^{k}}\left\|\mathbf{u}_{R}^{\varepsilon}\right\|_{H^{k}}+C \varepsilon\left\|n_{R}^{\varepsilon}\right\|_{H^{3}}\left\|\mathbf{u}_{R}^{\varepsilon}\right\|_{H^{k}} \\
& \leq C\left(1+\varepsilon C_{1}\right)\left\|n_{R}^{\varepsilon}\right\|_{H^{s^{\prime}}}^{2}+C\left\|\mathbf{u}_{R}^{\varepsilon}\right\|_{H^{k}}^{2},
\end{aligned}
$$

where $C_{1}=C_{1}(\varepsilon \tilde{C})$ is a constant depending on $\varepsilon \tilde{C}$.

Estimate of $I_{9}$. The estimate of $I_{9}$ in $(3.29)$ is similar to $I_{8}$. Recalling $(2.30)$, we have

$$
\left|I_{9}\right| \leq C \varepsilon^{2}+C C_{1} \varepsilon^{2}\left\|n_{R}^{\varepsilon}\right\|_{H^{k}}^{2}+\left\|\mathbf{u}_{R}^{\varepsilon}\right\|_{H^{k}}^{2} .
$$

Summarizing, we have

$$
\sum_{i=1}^{5}\left|I_{i}\right|+\left|I_{8}\right|+\left|I_{9}\right| \leq C C_{1}\left(1+\varepsilon\left\|\left(\mathbf{u}_{R}^{\varepsilon}, n_{R}^{\varepsilon}\right)\right\|_{H^{s^{\prime}}}\right)\left(1+\left\|\left(\mathbf{u}_{R}^{\varepsilon}, n_{R}^{\varepsilon}\right)\right\|_{H^{s^{\prime}}}^{2}\right) .
$$

Estimate of $I_{6}$. The estimate for the $I_{6}$ is not straightforward, and is very delicate since we need to use the structure of the remainder system $\mathrm{B.25}$ ) very carefully. By integration by parts, we have

$$
I_{6}=\frac{1}{\varepsilon}\left\langle\partial_{x}^{\alpha} \phi_{R}^{\varepsilon}, \bar{\nabla} \cdot \partial_{x}^{\alpha} \mathbf{u}_{R}^{\varepsilon}\right\rangle_{0},
$$

where $|\alpha|=k$. Taking $\partial_{x}^{\alpha}$ of 2.28a with $|\alpha|=k$, we have

$$
\begin{aligned}
\frac{1}{\varepsilon} \bar{\nabla} \cdot \partial_{x}^{\alpha} \mathbf{u}_{R}^{\varepsilon}= & \frac{1}{n}\left\{\frac{V \mathbf{e}_{1}-\mathbf{u}}{\varepsilon} \cdot \bar{\nabla} \partial_{x}^{\alpha} n_{R}^{\varepsilon}-\partial_{t} \partial_{x}^{\alpha} n_{R}^{\varepsilon}-\left[\partial_{x}^{\alpha}, \frac{\mathbf{u}}{\varepsilon}\right] \cdot \bar{\nabla} n_{R}^{\varepsilon}\right. \\
& \left.-\left[\partial_{x}^{\alpha}, \frac{n}{\varepsilon}\right] \bar{\nabla} \cdot \mathbf{u}_{R}^{\varepsilon}-\partial_{x}^{\alpha}\left(n_{R}^{\varepsilon} \nabla \cdot \widetilde{\mathbf{u}}+\mathbf{u}_{R}^{\varepsilon} \cdot \nabla \widetilde{n}+\varepsilon R_{n}\right)\right\} .
\end{aligned}
$$

Accordingly, $I_{6}$ is divided into five parts

$$
\begin{aligned}
I_{6}= & \left\langle\partial_{x}^{\alpha} \phi_{R}^{\varepsilon}, \frac{V \mathbf{e}_{1}-\mathbf{u}}{\varepsilon n} \cdot \bar{\nabla} \partial_{x}^{\alpha} n_{R}^{\varepsilon}\right\rangle_{0}-\left\langle\partial_{x}^{\alpha} \phi_{R}^{\varepsilon}, \frac{1}{n} \partial_{t} \partial_{x}^{\alpha} n_{R}^{\varepsilon}\right\rangle_{0} \\
& -\left\langle\partial_{x}^{\alpha} \phi_{R}^{\varepsilon}, \frac{1}{\varepsilon n}\left[\partial_{x}^{\alpha}, \mathbf{u}\right] \cdot \bar{\nabla} n_{R}^{\varepsilon}\right\rangle_{0}-\left\langle\partial_{x}^{\alpha} \phi_{R}^{\varepsilon}, \frac{1}{\varepsilon n}\left[\partial_{x}^{\alpha}, n\right] \bar{\nabla} \cdot \mathbf{u}_{R}^{\varepsilon}\right\rangle_{0} \\
& -\left\langle\partial_{x}^{\alpha} \phi_{R}^{\varepsilon}, \frac{1}{n} \partial_{x}^{\alpha}\left(n_{R}^{\varepsilon} \nabla \cdot \widetilde{\mathbf{u}}+\mathbf{u}_{R}^{\varepsilon} \cdot \nabla \widetilde{n}+\varepsilon R_{n}\right)\right\rangle_{0} \\
= & : I_{61}+\cdots+I_{65} .
\end{aligned}
$$

We first estimate $I_{63}-I_{65}$.

On the other hand, recalling [2.27), by commutator estimates in [14], we have

$$
\begin{aligned}
\left\|\left[\partial_{x}^{\alpha}, \mathbf{u}\right] \cdot \bar{\nabla} n_{R}^{\varepsilon}\right\|_{L^{2}} & \leq C\left(\left\|\bar{\nabla} n_{R}^{\varepsilon}\right\|_{\dot{H}^{k-1}}\|\nabla \mathbf{u}\|_{L^{\infty}}+\|\mathbf{u}\|_{\dot{H}^{k}}\left\|\bar{\nabla} n_{R}^{\varepsilon}\right\|_{L^{\infty}}\right) \\
& \leq C \varepsilon\left(\left\|n_{R}^{\varepsilon}\right\|_{\dot{H}^{k}}\left(1+\varepsilon\left\|\nabla \mathbf{u}_{R}^{\varepsilon}\right\|_{L^{\infty}}\right)+\left\|\nabla \mathbf{u}_{R}^{\varepsilon}\right\|_{L^{\infty}}\left(1+\varepsilon\left\|n_{R}^{\varepsilon}\right\|_{H^{k}}\right)\right) \\
& \leq C \varepsilon\left(1+\varepsilon\left\|_{R}^{\varepsilon}\right\|_{H^{s^{\prime}}}\right)\left(\left\|\mathbf{u}_{R}^{\varepsilon}\right\|_{H^{s^{\prime}}}+\left\|n_{R}^{\varepsilon}\right\|_{H^{s^{\prime}}}\right),
\end{aligned}
$$

where $k \leq s^{\prime}$ and $s^{\prime} \geq 3$. Therefore, from $\underline{3.2}$, we have

$$
\begin{aligned}
\left|I_{63}\right| & \leq C\left\|\partial_{x}^{\alpha} \phi_{R}^{\varepsilon}\right\|_{L^{2}}\left\|\left[\partial_{x}^{\alpha}, \mathbf{u}\right] \cdot \bar{\nabla} n_{R}^{\varepsilon}\right\|_{L^{2}} \\
& \leq C\left(1+\varepsilon^{2}\left\|\mathbf{u}_{R}^{\varepsilon}\right\|_{H^{s^{\prime}}}^{2}\right)\left\|\left(\mathbf{u}_{R}^{\varepsilon}, n_{R}^{\varepsilon}\right)\right\|_{H^{s^{\prime}}}^{2}+C\left\|\phi_{R}^{\varepsilon}\right\|_{H^{s^{\prime}}}^{2} .
\end{aligned}
$$


Similarly, we have

$$
\left\|\left[\partial_{x}^{\alpha}, n\right] \bar{\nabla} \cdot \mathbf{u}_{R}^{\varepsilon}\right\|_{L^{2}} \leq C \varepsilon\left(1+\varepsilon\left\|\mathbf{u}_{R}^{\varepsilon}\right\|_{H^{s^{\prime}}}\left(\left\|\mathbf{u}_{R}^{\varepsilon}\right\|_{H^{s^{\prime}}}+\left\|n_{R}^{\varepsilon}\right\|_{H^{s^{\prime}}}\right),\right.
$$

from which it follows that

$$
\left|I_{64}\right| \leq C\left(1+\varepsilon^{2}\left\|\mathbf{u}_{R}^{\varepsilon}\right\|_{H^{s^{\prime}}}^{2}\right)\left\|\left(\mathbf{u}_{R}^{\varepsilon}, n_{R}^{\varepsilon}\right)\right\|_{H^{s^{\prime}}}^{2}+C\left\|\phi_{R}^{\varepsilon}\right\|_{H^{s^{\prime}}}^{2} .
$$

By multiplicative estimates, we have

$$
\left\|\partial_{x}^{\alpha}\left(n_{R}^{\varepsilon} \nabla \cdot \widetilde{\mathbf{u}}+\mathbf{u}_{R}^{\varepsilon} \cdot \nabla \widetilde{n}+\varepsilon R_{n}\right)\right\| \leq C\left\|\left(\mathbf{u}_{R}^{\varepsilon}, n_{R}^{\varepsilon}\right)\right\|_{H^{s^{\prime}}}+C \varepsilon^{2} .
$$

It follows that

$$
\left|I_{65}\right| \leq C \varepsilon\left(1+\varepsilon^{2}\left\|\mathbf{u}_{R}^{\varepsilon}\right\|_{H^{s^{\prime}}}^{2}\right)\left\|\left(\mathbf{u}_{R}^{\varepsilon}, n_{R}^{\varepsilon}\right)\right\|_{H^{s^{\prime}}}^{2}+C \varepsilon\left\|\phi_{R}^{\varepsilon}\right\|_{H^{s^{\prime}}}^{2} .
$$

Summarizing (3.42), (3.44) and (3.46), we have

$$
\left|I_{63}+I_{64}+I_{65}\right| \leq C\left\|\phi_{R}^{\varepsilon}\right\|_{H^{s^{\prime}}}^{2}+C\left(1+\varepsilon^{2}\left\|\mathbf{u}_{R}^{\varepsilon}\right\|_{H^{s^{\prime}}}^{2}\right)\left\|\left(n_{R}^{\varepsilon}, \mathbf{u}_{R}^{\varepsilon}\right)\right\|_{H^{s^{\prime}}}^{2} .
$$

For clarity, we estimate $I_{61}$ and $I_{62}$ in Lemma 3.5 and 3.6 respectively.

We complete the proof of Lemma 3.4 by the following Lemma 3.5 and 3.6

Lemma 3.5. Let $\left(n_{R}^{\varepsilon}, \boldsymbol{u}_{R}^{\varepsilon}, \phi_{R}^{\varepsilon}\right)$ be a solution to (2.28) and $0 \leq k \leq s^{\prime}$ be an integer, then

$$
\left|I_{61}\right| \leq C\left(1+C_{1}\left(\sqrt{\varepsilon}\left\|\phi_{R}^{\varepsilon}\right\|_{H^{s^{\prime}}}\right)+\varepsilon^{2}\left\|\left(n_{R}^{\varepsilon}, \boldsymbol{u}_{R}^{\varepsilon}\right)\right\|_{H^{4}}^{2}\right)\left(1+\left\|\phi_{R}^{\varepsilon}\right\|_{H^{s^{\prime}}}^{2}+\varepsilon\left\|\bar{\nabla} \phi_{R}^{\varepsilon}\right\|_{H^{s^{\prime}}}^{2},\right.
$$

where $I_{61}$ is given in (3.40) and $|\alpha|=k \leq s^{\prime}$.

Proof. Let $\alpha$ be a multi-index such that $|\alpha|=k$. Taking $\partial_{x}^{\alpha}$ of $(2.28 \mathrm{c}$, we have

$$
\partial_{x}^{\alpha} n_{R}^{\varepsilon}=\partial_{x}^{\alpha} \phi_{R}^{\varepsilon}-\varepsilon \bar{\Delta} \partial_{x}^{\alpha} \phi_{R}^{\varepsilon}+\varepsilon \partial_{x}^{\alpha}\left(\phi^{(1)} \phi_{R}^{\varepsilon}\right)+\varepsilon^{3 / 2} \partial_{x}^{\alpha} R_{\phi} .
$$

Then $I_{61}$ in $\underline{3.40}$ is divided into

$$
\begin{aligned}
I_{61}= & \left\langle\partial_{x}^{\alpha} \phi_{R}^{\varepsilon}, \frac{V \mathbf{e}_{1}-\mathbf{u}}{\varepsilon n} \cdot \bar{\nabla} \partial_{x}^{\alpha} \phi_{R}^{\varepsilon}\right\rangle_{0}-\varepsilon\left\langle\partial_{x}^{\alpha} \phi_{R}^{\varepsilon}, \frac{V \mathbf{e}_{1}-\mathbf{u}}{\varepsilon n} \cdot \bar{\nabla} \bar{\Delta} \partial_{x}^{\alpha} \phi_{R}^{\varepsilon}\right\rangle_{0} \\
& +\varepsilon\left\langle\partial_{x}^{\alpha} \phi_{R}^{\varepsilon}, \frac{V \mathbf{e}_{1}-\mathbf{u}}{\varepsilon n} \cdot \bar{\nabla} \partial_{x}^{\alpha}\left(\phi^{(1)} \phi_{R}^{\varepsilon}\right)\right\rangle_{0}+\varepsilon^{3 / 2}\left\langle\partial_{x}^{\alpha} \phi_{R}^{\varepsilon}, \frac{V \mathbf{e}_{1}-\mathbf{u}}{\varepsilon n} \cdot \bar{\nabla} \partial_{x}^{\alpha} R_{\phi}\right\rangle_{0} \\
= & : I_{611}+I_{612}+I_{613}+I_{614} .
\end{aligned}
$$

Estimate of $I_{611}$. By integrating by parts, we have

$$
I_{611}=-\frac{1}{2}\left\langle\partial_{x}^{\alpha} \phi_{R}^{\varepsilon}, \bar{\nabla} \cdot\left(\frac{V \mathbf{e}_{1}-\mathbf{u}}{\varepsilon n}\right) \partial_{x}^{\alpha} \phi_{R}^{\varepsilon}\right\rangle_{0}
$$

By direct computation, we have

$$
\bar{\nabla} \cdot\left(\frac{V \mathbf{e}_{1}-\mathbf{u}}{\varepsilon n}\right)=-\frac{\left(V \mathbf{e}_{1}-\mathbf{u}\right)}{n^{2}} \cdot\left(\bar{\nabla} \widetilde{n}+\varepsilon \bar{\nabla} n_{R}^{\varepsilon}\right)-\frac{1}{n}\left(\bar{\nabla} \cdot \widetilde{\mathbf{u}}+\varepsilon \bar{\nabla} \cdot \mathbf{u}_{R}^{\varepsilon}\right) .
$$

By assumption (3.1), (3.2) and Sobolev embedding, we know that

$$
\begin{aligned}
\left\|\bar{\nabla} \cdot\left(\frac{V \mathbf{e}_{1}-\mathbf{u}}{\varepsilon n}\right)\right\|_{L^{\infty}} & \leq C\left(1+\varepsilon\left\|\bar{\nabla} n_{R}^{\varepsilon}\right\|_{\left.L^{\infty}+\varepsilon\left\|\bar{\nabla} \mathbf{u}_{R}^{\varepsilon}\right\|_{L^{\infty}}\right)}\right. \\
& \leq C\left(1+\varepsilon\left\|\left(n_{R}^{\varepsilon}, \mathbf{u}_{R}^{\varepsilon}\right)\right\|_{H^{3}}\right) .
\end{aligned}
$$


Using Hölder inequality in 3.49, we obtain

$$
\left|I_{611}\right| \leq C\left(1+\varepsilon^{2}\left\|\left(n_{R}^{\varepsilon}, \mathbf{u}_{R}^{\varepsilon}\right)\right\|_{H^{3}}^{2}\right)\left\|\partial_{x}^{\alpha} \phi_{R}^{\varepsilon}\right\|^{2} .
$$

Estimate of $I_{612}$. By integration by parts twice, we have

$$
\begin{aligned}
I_{612}= & \varepsilon\left\langle\bar{\nabla} \partial_{x}^{\alpha} \phi_{R}^{\varepsilon}, \frac{V \mathbf{e}_{1}-\mathbf{u}}{\varepsilon n} \bar{\Delta} \partial_{x}^{\alpha} \phi_{R}^{\varepsilon}\right\rangle_{0}+\varepsilon\left\langle\partial_{x}^{\alpha} \phi_{R}^{\varepsilon}, \bar{\nabla} \cdot\left(\frac{V \mathbf{e}_{1}-\mathbf{u}}{\varepsilon n}\right) \bar{\Delta} \partial_{x}^{\alpha} \phi_{R}^{\varepsilon}\right\rangle_{0} \\
= & -\varepsilon\left\langle\bar{\nabla} \partial_{x}^{\alpha} \phi_{R}^{\varepsilon}, \bar{\nabla}\left(\frac{V \mathbf{e}_{1}-\mathbf{u}}{\varepsilon n}\right) \bar{\nabla} \partial_{x}^{\alpha} \phi_{R}^{\varepsilon}\right\rangle_{0}-\frac{\varepsilon}{2}\left\langle\bar{\nabla} \partial_{x}^{\alpha} \phi_{R}^{\varepsilon}, \bar{\nabla} \cdot\left(\frac{V \mathbf{e}_{1}-\mathbf{u}}{\varepsilon n}\right) \bar{\nabla} \partial_{x}^{\alpha} \phi_{R}^{\varepsilon}\right\rangle_{0} \\
& -\varepsilon\left\langle\partial_{x}^{\alpha} \phi_{R}^{\varepsilon}, \overline{\nabla \nabla} \cdot\left(\frac{V \mathbf{e}_{1}-\mathbf{u}}{\varepsilon n}\right) \bar{\nabla} \partial_{x}^{\alpha} \phi_{R}^{\varepsilon}\right\rangle_{0} \\
= & : I_{6121}+I_{6122}+I_{6123} .
\end{aligned}
$$

From (3.50), the first two terms on the RHS can be bounded by

$$
I_{6121}, I_{6122} \leq C \varepsilon\left(1+\varepsilon^{2}\left\|\left(n_{R}^{\varepsilon}, \mathbf{u}_{R}^{\varepsilon}\right)\right\|_{H^{3}}^{2}\right)\left\|\bar{\nabla} \partial_{x}^{\alpha} \phi_{R}^{\varepsilon}\right\|^{2} .
$$

Similar to 3.50, we have

$$
\begin{aligned}
\left|\overline{\nabla \nabla} \cdot\left(\frac{V \mathbf{e}_{1}-\mathbf{u}}{\varepsilon n}\right)\right| \leq & C\left(1+\varepsilon\left|\bar{\nabla}^{2} n_{R}^{\varepsilon}\right|+\varepsilon\left|\overline{\nabla \nabla} \cdot \mathbf{u}_{R}^{\varepsilon}\right|+\varepsilon^{2}\left|\bar{\nabla} n_{R}^{\varepsilon}\right|\right. \\
& \left.+\varepsilon^{2}\left|\bar{\nabla} \cdot \mathbf{u}_{R}^{\varepsilon}\right|+\varepsilon^{3}\left|\bar{\nabla} n_{R}^{\varepsilon}\right|^{2}+\varepsilon^{3}\left|\bar{\nabla} \cdot \mathbf{u}_{R}^{\varepsilon}\right|^{2}\right),
\end{aligned}
$$

where we have used (2.30). Therefore, we have

$$
\begin{aligned}
\left|I_{6123}\right| \leq & C \varepsilon\left\|\partial_{x}^{\alpha} \phi_{R}^{\varepsilon}\right\|\left\|\bar{\nabla} \partial_{x}^{\alpha} \phi_{R}^{\varepsilon}\right\|+C \varepsilon^{2}\left\|\partial_{x}^{\alpha} \phi_{R}^{\varepsilon}\right\|_{L^{2}}\left(\left\|\bar{\nabla}^{2} n_{R}^{\varepsilon}\right\|_{L^{\infty}}+\left\|\bar{\nabla}^{2} \mathbf{u}_{R}^{\varepsilon}\right\|_{L^{\infty}}\right)\left\|\bar{\nabla} \partial_{x}^{\alpha} \phi_{R}^{\varepsilon}\right\| \\
& +C \varepsilon\left\|\partial_{x}^{\alpha} \phi_{R}^{\varepsilon}\right\|_{L^{2}}\left(1+\varepsilon^{3}\left\|\bar{\nabla} n_{R}^{\varepsilon}\right\|_{L^{\infty}}^{2}+\varepsilon^{3}\left\|\bar{\nabla} \cdot \mathbf{u}_{R}^{\varepsilon}\right\|_{L^{\infty}}^{2}\right)\left\|\bar{\nabla} \partial_{x}^{\alpha} \phi_{R}^{\varepsilon}\right\| \\
\leq & C\left\{\left\|\partial_{x}^{\alpha} \phi_{R}^{\varepsilon}\right\|^{2}+\varepsilon\left\|\bar{\nabla} \partial_{x}^{\alpha} \phi_{R}^{\varepsilon}\right\|^{2}\right\} \\
& +C\left\{\left\|\partial_{x}^{\alpha} \phi_{R}^{\varepsilon}\right\|_{L^{2}}^{2}+\varepsilon^{2}\left\|\left(n_{R}^{\varepsilon}, \mathbf{u}_{R}^{\varepsilon}\right)\right\|_{H^{4}}^{2}\left(\varepsilon\left\|\bar{\nabla} \partial_{x}^{\alpha} \phi_{R}^{\varepsilon}\right\|_{L^{2}}^{2}\right)\right\} \\
& +C\left\{\left(1+\varepsilon^{2}\left\|\left(n_{R}^{\varepsilon}, \mathbf{u}_{R}^{\varepsilon}\right)\right\|_{H^{3}}^{2}\right)\left(\left\|\partial_{x}^{\alpha} \phi_{R}^{\varepsilon}\right\|_{L^{2}}^{2}+\varepsilon\left\|\bar{\nabla} \partial_{x}^{\alpha} \phi_{R}^{\varepsilon}\right\|^{2}\right)\right\} \\
\leq & C\left(1+\varepsilon^{2}\left\|\left(n_{R}^{\varepsilon}, \mathbf{u}_{R}^{\varepsilon}\right)\right\|_{H^{4}}^{2}\right)\left(\left\|\partial_{x}^{\alpha} \phi_{R}^{\varepsilon}\right\|^{2}+\varepsilon\left\|\bar{\nabla} \partial_{x}^{\alpha} \phi_{R}^{\varepsilon}\right\|^{2}\right) .
\end{aligned}
$$

Therefore, combining (3.53), 3.55) and 3.52), we obtain

$$
\left|I_{612}\right| \leq C\left(1+\varepsilon^{2}\left\|\left(n_{R}^{\varepsilon}, \mathbf{u}_{R}^{\varepsilon}\right)\right\|_{H^{4}}^{2}\right)\left(\left\|\partial_{x}^{\alpha} \phi_{R}^{\varepsilon}\right\|^{2}+\varepsilon\left\|\bar{\nabla} \partial_{x}^{\alpha} \phi_{R}^{\varepsilon}\right\|^{2}\right) .
$$

Estimate of $I_{613}$. By integrating by parts, we have

$$
\begin{aligned}
I_{613} & =\left\langle\partial_{x}^{\alpha} \phi_{R}^{\varepsilon},\left(\frac{\left(V \mathbf{e}_{1}-\mathbf{u}\right) \phi^{(1)}}{n}\right) \cdot \bar{\nabla} \partial_{x}^{\alpha} \phi_{R}^{\varepsilon}\right\rangle_{0}+\left\langle\partial_{x}^{\alpha} \phi_{R}^{\varepsilon}, \frac{\left(V \mathbf{e}_{1}-\mathbf{u}\right)}{n}\left[\bar{\nabla} \partial_{x}^{\alpha}, \phi^{(1)}\right] \phi_{R}^{\varepsilon}\right\rangle_{0} \\
& =: I_{6131}+I_{6132} .
\end{aligned}
$$

By integrating by parts, we know

$$
I_{6131}=-\frac{1}{2}\left\langle\partial_{x}^{\alpha} \phi_{R}^{\varepsilon}, \bar{\nabla} \cdot\left(\frac{\left(V \mathbf{e}_{1}-\mathbf{u}\right) \phi^{(1)}}{n}\right) \partial_{x}^{\alpha} \phi_{R}^{\varepsilon}\right\rangle_{0} .
$$

Similar to 3.50, from 3.1], 3.2) and Sobolev embedding, we know that

$$
\left\|\bar{\nabla} \cdot\left(\frac{\left(V \mathbf{e}_{1}-\mathbf{u}\right) \phi^{(1)}}{n}\right)\right\|_{L^{\infty}} \leq C\left(1+\varepsilon \|\left(n_{R}^{\varepsilon}, \mathbf{u}_{R}^{\varepsilon} \|_{H^{3}}\right) .\right.
$$


Hence, using Hölder inequality, we obtain

$$
\left|I_{6131}\right| \leq C\left(1+\varepsilon^{2}\left\|\left(n_{R}^{\varepsilon}, \mathbf{u}_{R}^{\varepsilon}\right)\right\|_{H^{3}}^{2}\right)\left\|\partial_{x}^{\alpha} \phi_{R}^{\varepsilon}\right\|^{2} .
$$

On the other hand, by commutator estimate, we know

$$
\begin{aligned}
\left\|\left[\bar{\nabla} \partial_{x}^{\alpha}, \phi^{(1)}\right] \phi_{R}^{\varepsilon}\right\|_{L^{2}} & \leq C\left(\left\|\phi_{R}^{\varepsilon}\right\|_{H^{k}}\left\|\phi^{(1)}\right\|_{L^{\infty}}+\left\|\phi^{(1)}\right\|_{H^{k}}\left\|\phi_{R}^{\varepsilon}\right\|_{L^{\infty}}\right. \\
& \leq C\left\|\phi_{R}^{\varepsilon}\right\|_{H^{s^{\prime}}}
\end{aligned}
$$

It follows that

$$
\left|I_{6132}\right| \leq C\left\|\phi_{R}^{\varepsilon}\right\|_{H^{s^{\prime}}}^{2}
$$

Therefore, we have

$$
\left|I_{613}\right| \leq C\left(1+\varepsilon^{2}\left\|n_{R}^{\varepsilon}\right\|_{H^{3}}^{2}+\varepsilon^{2}\left\|\mathbf{u}_{R}^{\varepsilon}\right\|_{H^{3}}^{2}\right)\left\|\phi_{R}^{\varepsilon}\right\|_{H^{k}}^{2} .
$$

Estimate of I $_{614}$. By Hölder inequality and A.9) in Corollary A.1, we have

$$
\begin{aligned}
\left|I_{614}\right| & \leq \varepsilon^{1 / 2}\left\langle\partial_{x}^{\alpha} \phi_{R}^{\varepsilon}, \frac{V \mathbf{e}_{1}-\mathbf{u}}{n} \cdot \bar{\nabla} \partial_{x}^{\alpha} R_{\phi}\right\rangle_{0} \\
& \leq C\left\|\partial_{x}^{\alpha} \phi_{R}^{\varepsilon}\right\|^{2}+C_{1}\left(\sqrt{\varepsilon}\left\|\phi_{R}^{\varepsilon}\right\|_{H^{s^{\prime}}}\right)\left(1+\varepsilon\left\|\bar{\nabla} \phi_{R}^{\varepsilon}\right\|_{H^{s^{\prime}}}^{2}\right),
\end{aligned}
$$

where $|\alpha|=k \leq s^{\prime}$. From (3), adding (3.51), 3.56, (3.60) and (3.61) together, we obtain (3.47).

Lemma 3.6. Let $\left(n_{R}^{\varepsilon}, \boldsymbol{u}_{R}^{\varepsilon}, \phi_{R}^{\varepsilon}\right)$ be a solution to (B.25) and $0 \leq k \leq s^{\prime}$, then

$$
\begin{aligned}
I_{62} \leq & -\frac{1}{2} \frac{d}{d t} \int \frac{1+\varepsilon \phi^{(1)}+\varepsilon^{2} \phi_{R}^{\varepsilon}}{n}\left|\partial_{x}^{\alpha} \phi_{R}^{\varepsilon}\right|^{2}-\frac{1}{2} \frac{d}{d t} \int \frac{\varepsilon}{n}\left|\bar{\nabla} \partial_{x}^{\alpha} \phi_{R}^{\varepsilon}\right|^{2} \\
& +C C_{1}\left(C_{1}+\varepsilon\left\|\left(\boldsymbol{u}_{R}^{\varepsilon}, n_{R}^{\varepsilon}, \phi_{R}^{\varepsilon}\right)\right\|_{H^{s^{\prime}}}^{2}\right)\left\{1+\left\|\left(\boldsymbol{u}_{R}^{\varepsilon}, n_{R}^{\varepsilon}, \phi_{R}^{\varepsilon}\right)\right\|_{H^{s^{\prime}}}^{2}\right\} .
\end{aligned}
$$

where $|\alpha|=k \leq s^{\prime}$ and $I_{62}$ is given in (3.40).

Proof. Taking $\partial_{x}^{\alpha}$ of $2.28 \mathrm{c}$, we have

$$
\partial_{x}^{\alpha} n_{R}^{\varepsilon}=\partial_{x}^{\alpha} \phi_{R}^{\varepsilon}-\varepsilon \bar{\Delta} \partial_{x}^{\alpha} \phi_{R}^{\varepsilon}+\varepsilon \partial_{x}^{\alpha}\left(\phi^{(1)} \phi_{R}^{\varepsilon}\right)+\frac{\varepsilon^{2}}{2} \partial_{x}^{\alpha}\left[\left(\phi_{R}^{\varepsilon}\right)^{2}\right]+\varepsilon^{2} \partial_{x}^{\alpha} \bar{R}_{\phi} .
$$

Inserting this into $I_{62}$, we have

$$
\begin{aligned}
I_{62}= & -\left\langle\partial_{x}^{\alpha} \phi_{R}^{\varepsilon}, \frac{1}{n} \partial_{t} \partial_{x}^{\alpha} \phi_{R}^{\varepsilon}\right\rangle_{0}+\varepsilon\left\langle\partial_{x}^{\alpha} \phi_{R}^{\varepsilon}, \frac{1}{n} \partial_{t} \bar{\Delta} \partial_{x}^{\alpha} \phi_{R}^{\varepsilon}\right\rangle_{0} \\
& -\varepsilon\left\langle\partial_{x}^{\alpha} \phi_{R}^{\varepsilon}, \frac{1}{n} \partial_{t} \partial_{x}^{\alpha}\left(\phi^{(1)} \phi_{R}^{\varepsilon}\right)\right\rangle_{0}-\frac{\varepsilon^{2}}{2}\left\langle\partial_{x}^{\alpha} \phi_{R}^{\varepsilon}, \frac{1}{n} \partial_{t} \partial_{x}^{\alpha}\left[\left(\phi_{R}^{\varepsilon}\right)^{2}\right]\right\rangle_{0} \\
& -\varepsilon^{2}\left\langle\partial_{x}^{\alpha} \phi_{R}^{\varepsilon}, \frac{1}{n} \partial_{t} \partial_{x}^{\alpha} \bar{R}_{\phi}\right\rangle_{0} \\
= & : I_{621}+I_{622}+I_{623}+I_{624}+I_{625} .
\end{aligned}
$$

Estimate of $I_{621}$. By integration by parts in time, we obtain

$$
I_{621}=-\frac{1}{2} \frac{d}{d t}\left\langle\partial_{x}^{\alpha} \phi_{R}^{\varepsilon}, \frac{1}{n} \partial_{x}^{\alpha} \phi_{R}^{\varepsilon}\right\rangle_{0}+\frac{1}{2}\left\langle\partial_{x}^{\alpha} \phi_{R}^{\varepsilon}, \partial_{t}\left(\frac{1}{n}\right) \partial_{x}^{\alpha} \phi_{R}^{\varepsilon}\right\rangle_{0} .
$$

By direct computation, we have

$$
\partial_{t}\left(\frac{1}{n}\right)=-\frac{1}{n^{2}}\left(\varepsilon \partial_{t} \widetilde{n}+\varepsilon^{2} \partial_{t} n_{R}^{\varepsilon}\right)
$$


which yields that

$$
\left\|\partial_{t}\left(\frac{1}{n}\right)\right\|_{L^{\infty}} \leq C\left(\varepsilon+\varepsilon^{2}\left\|\partial_{t} n_{R}^{\varepsilon}\right\|_{L^{\infty}}\right),
$$

where we have used (3.1), 3.2) and Sobolev embedding. Therefore, from 3.64), we have

$$
\begin{aligned}
I_{621} & \leq-\frac{1}{2} \frac{d}{d t}\left\langle\partial_{x}^{\alpha} \phi_{R}^{\varepsilon}, \frac{1}{n} \partial_{x}^{\alpha} \phi_{R}^{\varepsilon}\right\rangle_{0}+C \varepsilon\left(1+\varepsilon\left\|\partial_{t} n_{R}^{\varepsilon}\right\|_{L^{\infty}}\right)\left\|\partial_{x}^{\alpha} \phi_{R}^{\varepsilon}\right\|^{2} \\
& \leq-\frac{1}{2} \frac{d}{d t}\left\langle\partial_{x}^{\alpha} \phi_{R}^{\varepsilon}, \frac{1}{n} \partial_{x}^{\alpha} \phi_{R}^{\varepsilon}\right\rangle_{0}+C\left(1+\varepsilon\left\|\left(n_{R}^{\varepsilon}, \mathbf{u}_{R}^{\varepsilon}\right)\right\|_{H^{3}}\right)\left\|\phi_{R}^{\varepsilon}\right\|_{H^{s^{\prime}}}^{2},
\end{aligned}
$$

where in the second inequality, we have used (3.12) in Lemma 3.2 .

Estimate of $I_{622}$. By first integrating by parts in space and then in time, we have

$$
\begin{aligned}
I_{622}= & -\varepsilon\left\langle\bar{\nabla} \partial_{x}^{\alpha} \phi_{R}^{\varepsilon}, \frac{1}{n} \partial_{t} \bar{\nabla} \partial_{x}^{\alpha} \phi_{R}^{\varepsilon}\right\rangle_{0}-\varepsilon\left\langle\partial_{x}^{\alpha} \phi_{R}^{\varepsilon}, \bar{\nabla}\left(\frac{1}{n}\right) \cdot \partial_{t} \bar{\nabla} \partial_{x}^{\alpha} \phi_{R}^{\varepsilon}\right\rangle_{0} \\
= & -\frac{1}{2} \frac{d}{d t}\left\langle\bar{\nabla} \partial_{x}^{\alpha} \phi_{R}^{\varepsilon}, \frac{\varepsilon}{n} \bar{\nabla} \partial_{x}^{\alpha} \phi_{R}^{\varepsilon}\right\rangle_{0} \\
& +\frac{\varepsilon}{2}\left\langle\bar{\nabla} \partial_{x}^{\alpha} \phi_{R}^{\varepsilon}, \partial_{t}\left(\frac{1}{n}\right) \bar{\nabla} \partial_{x}^{\alpha} \phi_{R}^{\varepsilon}\right\rangle_{0}-\varepsilon\left\langle\partial_{x}^{\alpha} \phi_{R}^{\varepsilon}, \bar{\nabla}\left(\frac{1}{n}\right) \cdot \partial_{t} \bar{\nabla} \partial_{x}^{\alpha} \phi_{R}^{\varepsilon}\right\rangle_{0} .
\end{aligned}
$$

From (3.65), the second term on the RHS of (3.67) is bounded by

$$
\left|\frac{1}{2}\left\langle\bar{\nabla} \partial_{x}^{\alpha} \phi_{R}^{\varepsilon}, \partial_{t}\left(\frac{\varepsilon}{n}\right) \bar{\nabla} \partial_{x}^{\alpha} \phi_{R}^{\varepsilon}\right\rangle_{0}\right| \leq C \varepsilon\left(\varepsilon+\varepsilon\left\|\varepsilon \partial_{t} n_{R}^{\varepsilon}\right\|_{L^{\infty}}\right)\left\|\bar{\nabla} \partial_{x}^{\alpha} \phi_{R}^{\varepsilon}\right\|^{2} .
$$

By Hölder inequality, the third term on the RHS of 3.67 is bounded by

$$
\begin{aligned}
\left|\varepsilon\left\langle\partial_{x}^{\alpha} \phi_{R}^{\varepsilon}, \bar{\nabla}\left(\frac{1}{n}\right) \cdot \partial_{t} \bar{\nabla} \partial_{x}^{\alpha} \phi_{R}^{\varepsilon}\right\rangle_{0}\right| & \leq C \varepsilon^{2}\left\|\partial_{x}^{\alpha} \phi_{R}^{\varepsilon}\right\|\left\|\partial_{t} \bar{\nabla} \partial_{x}^{\alpha} \phi_{R}^{\varepsilon}\right\|+C \varepsilon^{3}\left\|\nabla n_{R}^{\varepsilon}\right\|_{L^{\infty}}\left\|\partial_{x}^{\alpha} \phi_{R}^{\varepsilon}\right\|\left\|\partial_{t} \bar{\nabla} \partial_{x}^{\alpha} \phi_{R}^{\varepsilon}\right\| \\
& \leq C\left(1+\varepsilon\left\|\bar{\nabla} n_{R}^{\varepsilon}\right\|_{L^{\infty}}^{2}\right)\left\|\partial_{x}^{\alpha} \phi_{R}^{\varepsilon}\right\|^{2}+C \varepsilon^{3}\left\|\varepsilon \partial_{t} \bar{\nabla} \partial_{x}^{\alpha} \phi_{R}^{\varepsilon}\right\|^{2} \\
& \leq C\left(1+\varepsilon\left\|\bar{\nabla} n_{R}^{\varepsilon}\right\|_{L^{\infty}}^{2}\right)\left\|\partial_{x}^{\alpha} \phi_{R}^{\varepsilon}\right\|^{2}+C \varepsilon^{2}\left\|\varepsilon \partial_{t} \bar{\Delta} \phi_{R}^{\varepsilon}\right\|_{H^{k-1}}^{2},
\end{aligned}
$$

where we have used Corollary 3.2 and

$$
\left\|\bar{\nabla}\left(\frac{1}{n}\right)\right\|_{L^{\infty}} \leq C \varepsilon\left(1+\varepsilon\left\|\nabla n_{R}^{\varepsilon}\right\|_{L^{\infty}}\right) .
$$

Therefore, $I_{622}$ in (3.67) can be estimated as

$$
\begin{aligned}
I_{622} \leq & -\frac{1}{2} \frac{d}{d t}\left\langle\bar{\nabla} \partial_{x}^{\alpha} \phi_{R}^{\varepsilon}, \frac{\varepsilon}{n} \bar{\nabla} \partial_{x}^{\alpha} \phi_{R}^{\varepsilon}\right\rangle_{0} \\
& +C\left(1+\varepsilon\left\|\nabla n_{R}^{\varepsilon}\right\|_{L^{\infty}}^{2}+\varepsilon\left\|\varepsilon \partial_{t} n_{R}^{\varepsilon}\right\|_{L^{\infty}}^{2}\right)\left(\left\|\partial_{x}^{\alpha} \phi_{R}^{\varepsilon}\right\|^{2}+\varepsilon\left\|\bar{\nabla} \partial_{x}^{\alpha} \phi_{R}^{\varepsilon}\right\|^{2}\right) \\
& +C\left\{1+\left\|\left(\mathbf{u}_{R}^{\varepsilon}, n_{R}^{\varepsilon}, \phi_{R}^{\varepsilon}\right)\right\|_{H^{\delta+1}}^{2}\right\},
\end{aligned}
$$

where $\delta=\max \{2, k-1\}$ and we have used (3.24) in Corollary 3.1 Using Lemma3.1 and 3.2 we have

$$
\begin{aligned}
I_{622} \leq & -\frac{1}{2} \frac{d}{d t}\left\langle\bar{\nabla} \partial_{x}^{\alpha} \phi_{R}^{\varepsilon}, \frac{\varepsilon}{n} \bar{\nabla} \partial_{x}^{\alpha} \phi_{R}^{\varepsilon}\right\rangle_{0} \\
& +C\left(1+\varepsilon\left\|\left(\mathbf{u}_{R}^{\varepsilon}, n_{R}^{\varepsilon}, \phi_{R}^{\varepsilon}\right)\right\|_{H^{3}}^{2}\right)\left\{1+\left\|\left(\mathbf{u}_{R}^{\varepsilon}, n_{R}^{\varepsilon}, \phi_{R}^{\varepsilon}\right)\right\|_{H^{s^{s}}}^{2}\right\} .
\end{aligned}
$$

Estimate of $I_{623}$. By integration by parts in time, we obtain

$$
\begin{aligned}
I_{623}= & -\left\langle\partial_{x}^{\alpha} \phi_{R}^{\varepsilon}, \frac{\varepsilon \phi^{(1)}}{n} \partial_{t} \partial_{x}^{\alpha} \phi_{R}^{\varepsilon}\right\rangle_{0}-\left\langle\partial_{x}^{\alpha} \phi_{R}^{\varepsilon}, \frac{\varepsilon}{n}\left[\partial_{x}^{\alpha}, \phi^{(1)}\right] \partial_{t} \phi_{R}^{\varepsilon}\right\rangle_{0} \\
& -\left\langle\partial_{x}^{\alpha} \phi_{R}^{\varepsilon}, \frac{\varepsilon}{n} \partial_{x}^{\alpha}\left(\partial_{t} \phi^{(1)} \phi_{R}^{\varepsilon}\right)\right\rangle_{0} \\
= & : I_{6231}+I_{6232}+I_{6233},
\end{aligned}
$$


where $|\alpha|=k \leq s^{\prime}$. For the first on the RHS, by integration by parts, we have

$$
I_{6231}=-\frac{1}{2} \frac{d}{d t}\left\langle\partial_{x}^{\alpha} \phi_{R}^{\varepsilon}, \frac{\varepsilon \phi^{(1)}}{n} \partial_{x}^{\alpha} \phi_{R}^{\varepsilon}\right\rangle_{0}+\frac{1}{2}\left\langle\partial_{x}^{\alpha} \phi_{R}^{\varepsilon}, \partial_{t}\left(\frac{\varepsilon \phi^{(1)}}{n}\right) \partial_{x}^{\alpha} \phi_{R}^{\varepsilon}\right\rangle_{0} .
$$

By direct computation, we have

$$
\partial_{t}\left(\frac{\varepsilon \phi^{(1)}}{n}\right)=\frac{\varepsilon \partial_{t} \phi^{(1)}}{n}-\frac{\varepsilon \phi^{(1)}}{n^{2}}\left(\varepsilon \partial_{t} \tilde{n}+\varepsilon^{2} \partial_{t} n_{R}^{\varepsilon}\right),
$$

which yields that

$$
\left\|\partial_{t}\left(\frac{\varepsilon \phi^{(1)}}{n}\right)\right\|_{L^{\infty}} \leq C \varepsilon\left(1+\varepsilon^{2}\left\|\partial_{t} n_{R}^{\varepsilon}\right\|_{L^{\infty}}\right),
$$

where we have used (3.1), 3.2) and Sobolev embedding. Therefore, from (3.72), we have

$$
I_{6231} \leq-\frac{1}{2} \frac{d}{d t}\left\langle\partial_{x}^{\alpha} \phi_{R}^{\varepsilon}, \frac{\varepsilon \phi^{(1)}}{n} \partial_{x}^{\alpha} \phi_{R}^{\varepsilon}\right\rangle_{0}+C \varepsilon\left(1+\varepsilon^{2}\left\|\partial_{t} n_{R}^{\varepsilon}\right\|_{L^{\infty}}\right)\left\|\partial_{x}^{\alpha} \phi_{R}^{\varepsilon}\right\|^{2} .
$$

By commutator estimates, we have

$$
\begin{aligned}
\left\|\left[\partial_{x}^{\alpha}, \phi^{(1)}\right] \partial_{t} \phi_{R}^{\varepsilon}\right\|_{L^{2}} & \leq C\left(\left\|\partial_{t} \phi_{R}^{\varepsilon}\right\|_{H^{k-1}}\left\|\phi^{(1)}\right\|_{L^{\infty}}+\left\|\partial_{t} \phi_{R}^{\varepsilon}\right\|_{L^{\infty}}\left\|\phi^{(1)}\right\|_{H^{k-1}}\right) \\
& \leq C\left(\left\|\partial_{t} \phi_{R}^{\varepsilon}\right\|_{H^{k-1}}+\left\|\partial_{t} \phi_{R}^{\varepsilon}\right\|_{H^{2}}\right) .
\end{aligned}
$$

Therefore, by using (3.24) in Corollary 3.1, we have for the second term on the RHS of (3.71), we have

$$
\begin{aligned}
I_{6232} & \leq C\left\|\partial_{x}^{\alpha} \phi_{R}^{\varepsilon}\right\|^{2}+C \varepsilon^{2}\left\|\partial_{t} \phi_{R}^{\varepsilon}\right\|_{H^{\delta}}^{2} \\
& \leq C\left\{1+\|\left(n_{R}^{\varepsilon}, \mathbf{u}_{R}^{\varepsilon}, \phi_{R}^{\varepsilon} \|_{H^{\delta+1}}^{2}\right\},\right.
\end{aligned}
$$

where $\delta=\max \{2, k-1\}$. By multiplicative estimates, we have

$$
\begin{aligned}
\left\|\partial_{x}^{\alpha}\left(\partial_{t} \phi^{(1)} \phi_{R}^{\varepsilon}\right)\right\|_{L^{2}} & \leq C\left(\left\|\phi_{R}^{\varepsilon}\right\|_{H^{k}}\left\|\partial_{t} \phi^{(1)}\right\|_{L^{\infty}}+\left\|\phi_{R}^{\varepsilon}\right\|_{L^{\infty}}\left\|\partial_{t} \phi^{(1)}\right\|_{H^{k}}\right) \\
& \leq C\left(\left\|\phi_{R}^{\varepsilon}\right\|_{H^{k}}+\left\|\phi_{R}^{\varepsilon}\right\|_{H^{2}}\right) .
\end{aligned}
$$

Then, for the third term on the RHS of 3.71, we have

$$
I_{6233} \leq C \varepsilon\left\|\phi_{R}^{\varepsilon}\right\|_{H^{s^{\prime}}}^{2},
$$

where $|\alpha|=k \leq s^{\prime}$. Therefore, adding 3.73, 3.74 and 3.75) together and using (3.12) in Lemma 3.2 we have

$$
\begin{aligned}
I_{623} \leq & -\frac{1}{2} \frac{d}{d t}\left\langle\partial_{x}^{\alpha} \phi_{R}^{\varepsilon}, \frac{\varepsilon \phi^{(1)}}{n} \partial_{x}^{\alpha} \phi_{R}^{\varepsilon}\right\rangle_{0} \\
& +C\left(1+\varepsilon^{2}\left\|\left(n_{R}^{\varepsilon}, \mathbf{u}_{R}^{\varepsilon}\right)\right\|_{H^{3}}^{2}\right)\left\{1+\left\|\left(\mathbf{u}_{R}^{\varepsilon}, n_{R}^{\varepsilon}, \phi_{R}^{\varepsilon}\right)\right\|_{H^{s^{\prime}}}^{2}\right\},
\end{aligned}
$$

where $\delta=\max \{2, k-1\}, k \leq s^{\prime}$ and $s^{\prime} \geq 3$.

Estimate of $I_{624}$. We have

$$
\begin{aligned}
I_{624} & =-\varepsilon^{2}\left\langle\partial_{x}^{\alpha} \phi_{R}^{\varepsilon}, \frac{\phi_{R}^{\varepsilon}}{n} \partial_{t} \partial_{x}^{\alpha} \phi_{R}^{\varepsilon}\right\rangle_{0}-\varepsilon^{2}\left\langle\partial_{x}^{\alpha} \phi_{R}^{\varepsilon}, \frac{1}{n}\left[\partial_{x}^{\alpha}, \phi_{R}^{\varepsilon}\right] \partial_{t} \phi_{R}^{\varepsilon}\right\rangle_{0} \\
& =: I_{6241}+I_{6242}
\end{aligned}
$$

By integrating by parts in time, we have

$$
I_{6241}=-\frac{1}{2} \frac{d}{d t} \int\left(\frac{\varepsilon^{2} \phi_{R}^{\varepsilon}}{n}\right)\left|\partial_{x}^{\alpha} \phi_{R}^{\varepsilon}\right|^{2} d x-\frac{1}{2} \int \partial_{t}\left(\frac{\varepsilon^{2} \phi_{R}^{\varepsilon}}{n}\right)\left|\partial_{x}^{\alpha} \phi_{R}^{\varepsilon}\right|^{2} d x .
$$


From (2.27), we have

$$
\begin{aligned}
\left\|\partial_{t}\left(\frac{\varepsilon^{2} \phi_{R}^{\varepsilon}}{n}\right)\right\|_{L^{\infty}} & \leq C \varepsilon^{2}\left(\left\|\partial_{t} \phi_{R}^{\varepsilon}\right\|_{L^{\infty}}+\varepsilon\left\|\partial_{t} \widetilde{n}\right\|_{L^{\infty}}+\varepsilon^{2}\left\|\partial_{t} n_{R}^{\varepsilon}\right\|_{L^{\infty}}\right) \\
& \leq C \varepsilon^{2}\left(\varepsilon+\left\|\partial_{t} \phi_{R}^{\varepsilon}\right\|_{H^{2}}+\varepsilon^{2}\left\|\partial_{t} n_{R}^{\varepsilon}\right\|_{H^{2}}\right) .
\end{aligned}
$$

From Lemma 3.2 and Corollary 3.1 it follows

$$
\left\|\partial_{t}\left(\frac{\varepsilon^{2} \phi_{R}^{\varepsilon}}{n}\right)\right\|_{L^{\infty}} \leq C \varepsilon\left(1+\left\|\mathbf{u}_{R}^{\varepsilon}\right\|_{H^{3}}+\left\|n_{R}^{\varepsilon}\right\|_{H^{3}}+\left\|\phi_{R}^{\varepsilon}\right\|_{H^{3}}\right) .
$$

Hence, we obtain

$$
I_{6241} \leq-\frac{1}{2} \frac{d}{d t} \int\left(\frac{\varepsilon^{2} \phi_{R}^{\varepsilon}}{n}\right)\left|\partial_{x}^{\alpha} \phi_{R}^{\varepsilon}\right|^{2} d x+C \varepsilon\left(1+\left\|\left(\mathbf{u}_{R}^{\varepsilon}, n_{R}^{\varepsilon}, \phi_{R}^{\varepsilon}\right)\right\|_{H^{3}}\right)\left\|\phi_{R}^{\varepsilon}\right\|_{H^{k}}^{2} .
$$

On the other hand, by commutator estimate, we have

$$
\begin{aligned}
\left\|\left[\partial_{x}^{\alpha}, \phi_{R}^{\varepsilon}\right] \partial_{t} \phi_{R}^{\varepsilon}\right\|_{L^{2}} \leq & C\left(\left\|\partial_{t} \phi_{R}^{\varepsilon}\right\|_{H^{k-1}}\left\|\phi_{R}^{\varepsilon}\right\|_{L^{\infty}}+\left\|\partial_{t} \phi_{R}^{\varepsilon}\right\|_{L^{\infty}}\left\|\phi_{R}^{\varepsilon}\right\|_{H^{k}}\right) \\
\leq & C\left\{\left\|\phi_{R}^{\varepsilon}\right\|_{H^{2}}\left(1+\left\|\partial_{t} n_{R}^{\varepsilon}\right\|_{H^{k-1}}+\left\|\phi_{R}^{\varepsilon}\right\|_{H^{k-1}}\right)\right. \\
& \left.+\left\|\phi_{R}^{\varepsilon}\right\|_{H^{k}}\left(1+\left\|\partial_{t} n_{R}^{\varepsilon}\right\|_{H^{2}}+\left\|\phi_{R}^{\varepsilon}\right\|_{H^{2}}\right)\right\}
\end{aligned}
$$

where we have used Lemma3.3. Since $k \leq s^{\prime}$, by using Lemma 3.2, we have

$$
\varepsilon\left\|\left[\partial_{x}^{\alpha}, \phi_{R}^{\varepsilon}\right] \partial_{t} \phi_{R}^{\varepsilon}\right\|_{L^{2}} \leq C\left\|\phi_{R}^{\varepsilon}\right\|_{H^{s^{\prime}}}\left(1+\left\|\left(\mathbf{u}_{R}^{\varepsilon}, n_{R}^{\varepsilon}, \phi_{R}^{\varepsilon}\right)\right\|_{H^{s^{\prime}}}\right) .
$$

It then follows that

$$
\left|I_{6242}\right| \leq C\left(1+\varepsilon\left\|\left(\mathbf{u}_{R}^{\varepsilon}, n_{R}^{\varepsilon}, \phi_{R}^{\varepsilon}\right)\right\|_{H^{s^{\prime}}}\right)\left\|\phi_{R}^{\varepsilon}\right\|_{H^{s^{\prime}}}^{2}
$$

Combining (3.79) and (3.78), we have

$$
I_{624} \leq-\frac{1}{2} \frac{d}{d t} \int\left(\frac{\varepsilon^{2} \phi_{R}^{\varepsilon}}{n}\right)\left|\partial_{x}^{\alpha} \phi_{R}^{\varepsilon}\right|^{2} d x+C\left(1+\varepsilon\left\|\left(\mathbf{u}_{R}^{\varepsilon}, n_{R}^{\varepsilon}, \phi_{R}^{\varepsilon}\right)\right\|_{H^{s^{\prime}}}\right)\left\|\phi_{R}^{\varepsilon}\right\|_{H^{s^{\prime}}}^{2} .
$$

Estimate of $I_{625}$. By (2.23) in Lemma2.1, we have

$$
\begin{aligned}
I_{625} & \leq C\left\|\partial_{x}^{\alpha} \phi_{R}^{\varepsilon}\right\|^{2}+\varepsilon^{4}\left\|\partial_{t} \partial_{x}^{\alpha} \bar{R}_{\phi}\right\|^{2} \\
& \leq C\left\|\partial_{x}^{\alpha} \phi_{R}^{\varepsilon}\right\|^{2}+C_{1}\left(\sqrt{\varepsilon}\left\|\phi_{R}^{\varepsilon}\right\|_{H^{\delta}}\right)\left(1+\varepsilon^{2}\left\|\varepsilon \partial_{t} \phi_{R}^{\varepsilon}\right\|_{H^{k}}^{2}\right),
\end{aligned}
$$

where $\delta=\max \{2, k-1\}$ in Lemma2.1. Furthermore,

$$
\begin{aligned}
\varepsilon^{2}\left\|\varepsilon \partial_{t} \phi_{R}^{\varepsilon}\right\|_{H^{k}}^{2} & \leq \varepsilon\left\|\varepsilon \partial_{t}\left(\varepsilon^{1 / 2} \nabla\right) \phi_{R}^{\varepsilon}\right\|_{H^{k-1}}^{2}+\varepsilon^{2}\left\|\varepsilon \partial_{t} \phi_{R}^{\varepsilon}\right\|_{H^{k-1}}^{2} \\
& \leq \varepsilon\left\|\varepsilon \partial_{t} \bar{\nabla} \phi_{R}^{\varepsilon}\right\|_{H^{k-1}}^{2}+\varepsilon^{2}\left\|\varepsilon \partial_{t} \phi_{R}^{\varepsilon}\right\|_{H^{k-1}}^{2} \\
& \leq C\left\|\varepsilon \partial_{t} n_{R}^{\varepsilon}\right\|_{H^{k-1}}^{2}+C \varepsilon^{3}\left\|\phi_{R}^{\varepsilon}\right\|_{H^{k-1}}^{2}+C C_{0}^{2} \varepsilon^{3},
\end{aligned}
$$

where we have used (3.23). By Corollary 3.1 when $0<\varepsilon<\varepsilon_{1}$, we have

$$
\varepsilon^{2}\left\|\varepsilon \partial_{t} \phi_{R}^{\varepsilon}\right\|_{H^{k}}^{2} \leq C\left\{1+\left\|\mathbf{u}_{R}^{\varepsilon}\right\|_{H^{s^{\prime}}}^{2}+\left\|n_{R}^{\varepsilon}\right\|_{H^{s^{\prime}}}^{2}+\varepsilon^{3}\left\|\phi_{R}^{\varepsilon}\right\|_{H^{k-1}}^{2}\right\} .
$$

It then follows from 3.80 that

$$
I_{625} \leq C_{1}\left(\sqrt{\varepsilon}\left\|\phi_{R}^{\varepsilon}\right\|_{H^{\delta}}\right)\left\{1+\left\|\mathbf{u}_{R}^{\varepsilon}\right\|_{H^{s^{\prime}}}^{2}+\left\|n_{R}^{\varepsilon}\right\|_{H^{s^{\prime}}}^{2}+\left\|\phi_{R}^{\varepsilon}\right\|_{H^{s^{s}}}^{2}\right\},
$$

where $\delta=\max \{2, k-1\} \leq s^{\prime}-1$.

Summarizing, from 3.63), 3.66, 3.70, 3.76), 3.79) and 3.81, we have 3.62). The proof is complete. 
Lemma 3.7. Let $s^{\prime} \geq 4$ be integer and $\left(n_{R}^{\varepsilon}, \boldsymbol{u}_{R}^{\varepsilon}, \phi_{R}^{\varepsilon}\right)$ be a solution to (2.28). The for any $0 \leq k \leq s^{\prime}$, there holds

$$
\frac{1}{2} \frac{d}{d t} \int \frac{T_{i}}{n^{2}}\left|\partial_{x}^{\alpha} n_{R}^{\varepsilon}\right|^{2} d x \leq C\left(\varepsilon+\varepsilon\left\|\left(\boldsymbol{u}_{R}^{\varepsilon}, n_{R}^{\varepsilon}\right)\right\|_{H^{s^{\prime}}}\right)\left\{1+\left\|\left(n_{R}^{\varepsilon}, \boldsymbol{u}_{R}^{\varepsilon}\right)\right\|_{H^{s^{\prime}}}^{2}\right\}+J_{31},
$$

where $\alpha$ is any multi-index with $|\alpha|=k$ and

$$
J_{31}=-T_{i}\left\langle\left(\bar{\nabla} \cdot \partial_{x}^{\alpha} \boldsymbol{u}_{R}^{\varepsilon}\right), \frac{1}{\varepsilon n} \partial_{x}^{\alpha} n_{R}^{\varepsilon}\right\rangle_{0} .
$$

Proof. Let $\alpha$ be a multi-index with $|\alpha|=k \leq s^{\prime}$. We take $\partial_{x}^{\alpha}$ of $\underline{2.28 \mathrm{a}}$, and then take inner product of $\frac{T_{i}}{n^{2}} \partial_{x}^{\alpha} n_{R}^{\varepsilon}$ in $L^{2}$. Integrating in $t$, we obtain

$$
\begin{aligned}
\frac{1}{2} \frac{d}{d t} \int \frac{T_{i}}{n^{2}}\left|\partial_{x}^{\alpha} n_{R}^{\varepsilon}\right|^{2} d x= & \frac{1}{2} \int \partial_{t}\left(\frac{T_{i}}{n^{2}}\right)\left|\partial_{x}^{\alpha} n_{R}^{\varepsilon}\right|^{2} d x+\left\langle\partial_{x}^{\alpha}\left(\frac{V \mathbf{e}_{1}-\mathbf{u}}{\varepsilon} \cdot \bar{\nabla} n_{R}^{\varepsilon}\right), \frac{T_{i}}{n^{2}} \partial_{x}^{\alpha} n_{R}^{\varepsilon}\right\rangle_{0} \\
& -\left\langle\partial_{x}^{\alpha}\left(\frac{n}{\varepsilon} \bar{\nabla} \cdot \mathbf{u}_{R}^{\varepsilon}\right), \frac{T_{i}}{n^{2}} \partial_{x}^{\alpha} n_{R}^{\varepsilon}\right\rangle_{0}-\left\langle\partial_{x}^{\alpha}\left(n_{R}^{\varepsilon} \bar{\nabla} \cdot \widetilde{\mathbf{u}}\right), \frac{T_{i}}{n^{2}} \partial_{x}^{\alpha} n_{R}^{\varepsilon}\right\rangle_{0} \\
& -\left\langle\partial_{x}^{\alpha}\left(\mathbf{u}_{R}^{\varepsilon} \cdot \bar{\nabla} \widetilde{n}\right), \frac{T_{i}}{n^{2}} \partial_{x}^{\alpha} n_{R}^{\varepsilon}\right\rangle_{0}-\left\langle\partial_{x}^{\alpha}\left(\varepsilon R_{n}\right), \frac{T_{i}}{n^{2}} \partial_{x}^{\alpha} n_{R}^{\varepsilon}\right\rangle_{0} \\
= & J_{1}+\cdots+J_{6} .
\end{aligned}
$$

Estimate of $J_{1}$. By direct computation, we have

$$
\partial_{t}\left(\frac{T_{i}}{n^{2}}\right)=-2 \frac{1}{n^{3}}\left(\varepsilon \partial_{t} \tilde{n}+\varepsilon^{2} \partial_{t} n_{R}^{\varepsilon}\right)
$$

which yields that

$$
\left\|\partial_{t}\left(\frac{T_{i}}{n^{2}}\right)\right\|_{L^{\infty}} \leq C\left(\varepsilon+\varepsilon\left\|\varepsilon \partial_{t} n_{R}^{\varepsilon}\right\|_{L^{\infty}}\right),
$$

where we have used (3.1) and (3.2). By Lemma 3.2 and 3.1 and Sobolev embedding, we have

$$
\left\|\varepsilon \partial_{t} n_{R}^{\varepsilon}\right\|_{L^{\infty}} \leq\left\|\varepsilon \partial_{t} n_{R}^{\varepsilon}\right\|_{H^{2}} \leq C\left\{1+\left\|\mathbf{u}_{R}^{\varepsilon}\right\|_{H^{3}}+\left\|n_{R}^{\varepsilon}\right\|_{H^{3}}\right\} .
$$

Therefore,

$$
\left|J_{1}\right| \leq C\left(\varepsilon+\varepsilon\left\|\left(\mathbf{u}_{R}^{\varepsilon}, n_{R}^{\varepsilon}\right)\right\|_{H^{3}}\right)\left\|n_{R}^{\varepsilon}\right\|_{H^{k}}^{2} .
$$

Estimate of $J_{2}$. Expanding the expression of $J_{2}$, we have

$$
J_{2}=\left\langle\partial_{x}^{\alpha}\left(\frac{V}{\varepsilon} \cdot \partial_{x_{1}} n_{R}^{\varepsilon}\right), \frac{T_{i}}{n^{2}} \partial_{x}^{\alpha} n_{R}^{\varepsilon}\right\rangle_{0}-\left\langle\partial_{x}^{\alpha}\left(\frac{\mathbf{u}}{\varepsilon} \cdot \bar{\nabla} n_{R}^{\varepsilon}\right), \frac{T_{i}}{n^{2}} \partial_{x}^{\alpha} n_{R}^{\varepsilon}\right\rangle_{0}=: J_{21}+J_{22} .
$$

Similar to 3.85, we have

$$
\begin{aligned}
\left\|\partial_{x_{1}}\left(\frac{T_{i}}{n^{2}}\right)\right\|_{L^{\infty}} & \leq C\left(\varepsilon+\varepsilon^{2}\left\|\partial_{x_{1}} n_{R}^{\varepsilon}\right\|_{L^{\infty}}\right) \\
& \leq C\left(\varepsilon+\varepsilon^{2}\left\|n_{R}^{\varepsilon}\right\|_{H^{3}}\right) .
\end{aligned}
$$

Hence, by integrating by parts, we have

$$
\begin{aligned}
\left|J_{21}\right| & =\left.\left|-\frac{V}{2} \int \partial_{x_{1}}\left(\frac{T_{i}}{\varepsilon n^{2}}\right)\right| \partial_{x}^{\alpha} n_{R}^{\varepsilon}\right|^{2} d x \mid \\
& \leq C\left(1+\varepsilon\left\|n_{R}^{\varepsilon}\right\|_{H^{3}}\right)\left\|n_{R}^{\varepsilon}\right\|_{H^{k}}^{2} .
\end{aligned}
$$


Next, we estimate $J_{22}$ in 3.87. We have

$$
J_{22}=-\left\langle\left[\partial_{x}^{\alpha}, \frac{\mathbf{u}}{\varepsilon}\right] \bar{\nabla} n_{R}^{\varepsilon}, \frac{T_{i}}{n^{2}} \partial_{x}^{\alpha} n_{R}^{\varepsilon}\right\rangle_{0}-\left\langle\frac{\mathbf{u}}{\varepsilon} \partial_{x}^{\alpha} \bar{\nabla} n_{R}^{\varepsilon}, \frac{T_{i}}{n^{2}} \partial_{x}^{\alpha} n_{R}^{\varepsilon}\right\rangle_{0}=: J_{221}+J_{222} .
$$

By commutator estimate, we have

$$
\begin{aligned}
\left\|\left[\partial_{x}^{\alpha}, \frac{\mathbf{u}}{\varepsilon}\right] \bar{\nabla} n_{R}^{\varepsilon}\right\|_{L^{2}} & \leq C\left(\left\|\bar{\nabla} n_{R}^{\varepsilon}\right\|_{H^{k-1}}\left\|\nabla\left(\frac{\mathbf{u}}{\varepsilon}\right)\right\|_{L^{\infty}}+\left\|\bar{\nabla} n_{R}^{\varepsilon}\right\|_{L^{\infty}}\left\|\frac{\mathbf{u}}{\varepsilon}\right\|_{H^{k}}\right) \\
& \leq C\left\{\left\|n_{R}^{\varepsilon}\right\|_{H^{k}}\left(\|\widetilde{\mathbf{u}}\|_{H^{3}}+\varepsilon\left\|\mathbf{u}_{R}^{\varepsilon}\right\|_{H^{3}}\right)+\left\|n_{R}^{\varepsilon}\right\|_{H^{3}}\left(\|\widetilde{\mathbf{u}}\|_{H^{k}}+\varepsilon\left\|\mathbf{u}_{R}^{\varepsilon}\right\|_{H^{k}}\right)\right\} \\
& \leq C\left(1+\varepsilon\left\|\mathbf{u}_{R}^{\varepsilon}\right\|_{H^{s^{\prime}}}\right)\left\|n_{R}^{\varepsilon}\right\|_{H^{s^{\prime}}}
\end{aligned}
$$

where we have used $k \leq s^{\prime}, s^{\prime} \geq 3$ and 2.27). Therefore, we have

$$
\left|J_{221}\right| \leq C\left(1+\varepsilon\left\|\mathbf{u}_{R}^{\varepsilon}\right\|_{H^{s^{\prime}}}\right)\left\|n_{R}^{\varepsilon}\right\|_{H^{s^{\prime}}}^{2} .
$$

On the other hand, from (3.2) and (2.24), by Hölder inequality, we know

$$
\begin{aligned}
\left\|\bar{\nabla}\left(\frac{\mathbf{u}}{\varepsilon n^{2}}\right)\right\|_{L^{\infty}} & \leq\left\|\frac{\bar{\nabla} \widetilde{\mathbf{u}}+\varepsilon \bar{\nabla} \mathbf{u}_{R}^{\varepsilon}}{n^{2}}\right\|_{L^{\infty}}+2\left\|\mathbf{u} \frac{\bar{\nabla} \widetilde{n}+\varepsilon \bar{\nabla} n_{R}^{\varepsilon}}{n^{3}}\right\|_{L^{\infty}} \\
& \leq C+C \varepsilon\left(\left\|\bar{\nabla} \mathbf{u}_{R}^{\varepsilon}\right\|_{L^{\infty}}+\left\|\bar{\nabla} n_{R}^{\varepsilon}\right\|_{L^{\infty}}\right) \\
& \leq C+C \varepsilon\left(\left\|\mathbf{u}_{R}^{\varepsilon}\right\|_{H^{3}}+\left\|n_{R}^{\varepsilon}\right\|_{H^{3}}\right) .
\end{aligned}
$$

By integrating by parts, we obtain

$$
\begin{aligned}
\left|J_{222}\right| & =\left|\frac{T_{i}}{2}\left\langle\bar{\nabla}\left(\frac{\mathbf{u}}{\varepsilon n^{2}}\right) \partial_{x}^{\alpha} n_{R}^{\varepsilon}, \partial_{x}^{\alpha} n_{R}^{\varepsilon}\right\rangle_{0}\right| \\
& \leq C\left(1+\varepsilon\left(\left\|\mathbf{u}_{R}^{\varepsilon}\right\|_{H^{3}}+\left\|n_{R}^{\varepsilon}\right\|_{H^{3}}\right)\right)\left\|\partial_{x}^{\alpha} n_{R}^{\varepsilon}\right\|^{2} .
\end{aligned}
$$

Adding the estimates 3.88, 3.89) and 3.90 together, we have

$$
\left|J_{2}\right| \leq C\left(1+\varepsilon\left(\left\|\mathbf{u}_{R}^{\varepsilon}\right\|_{H^{s^{\prime}}}+\left\|n_{R}^{\varepsilon}\right\|_{H^{s^{\prime}}}\right)\right)\left\|n_{R}^{\varepsilon}\right\|_{H^{s^{\prime}}}^{2},
$$

where $k \leq s^{\prime}$ and $s^{\prime} \geq 3$.

Estimate of $J_{4}$. For $J_{4}$ in 3.84), we have

$$
\left|J_{4}\right| \leq C\left\|n_{R}^{\varepsilon}\right\|_{H^{k}}^{2}
$$

Estimate of $\mathrm{J}_{5}$. We have

$$
\left|J_{5}\right| \leq C\left\|\mathbf{u}_{R}^{\varepsilon}\right\|_{H^{k}}^{2}+C\left\|n_{R}^{\varepsilon}\right\|_{H^{k}}^{2}
$$

Estimate of $J_{6}$. We have

$$
\left|J_{6}\right| \leq C \varepsilon^{2}+C\left\|n_{R}^{\varepsilon}\right\|_{H^{k}}^{2}
$$

Estimate of $J_{3}$ in (3.84. $J_{3}$ can be written in the commutator form

$$
\begin{aligned}
J_{3} & =-T_{i}\left\langle\left(\bar{\nabla} \cdot \partial_{x}^{\alpha} \mathbf{u}_{R}^{\varepsilon}\right), \frac{1}{\varepsilon n} \partial_{x}^{\alpha} n_{R}^{\varepsilon}\right\rangle_{0}-T_{i}\left\langle\left[\partial_{x}^{\alpha}, \frac{n}{\varepsilon}\right] \bar{\nabla} \cdot \mathbf{u}_{R}^{\varepsilon}, \frac{1}{n^{2}} \partial_{x}^{\alpha} n_{R}^{\varepsilon}\right\rangle_{0} \\
& =J_{31}+J_{32} .
\end{aligned}
$$

By commutator estimate, we have

$$
\begin{aligned}
\left\|\left[\partial_{x}^{\alpha}, \frac{n}{\varepsilon}\right] \bar{\nabla} \cdot \mathbf{u}_{R}^{\varepsilon}\right\|_{L^{2}} & \leq C\left(\left\|\bar{\nabla} \cdot \mathbf{u}_{R}^{\varepsilon}\right\|_{\dot{H}^{k-1}}\left\|\nabla\left(\frac{n}{\varepsilon}\right)\right\|_{L^{\infty}}+\left\|\bar{\nabla} \cdot \mathbf{u}_{R}^{\varepsilon}\right\|_{L^{\infty}}\left\|\frac{n}{\varepsilon}\right\|_{\dot{H}^{k}}\right) \\
& \leq C\left(1+\varepsilon\left\|n_{R}^{\varepsilon}\right\|_{H^{s^{\prime}}}\right)\left\|\mathbf{u}_{R}^{\varepsilon}\right\|_{H^{s^{\prime}}} .
\end{aligned}
$$


Therefore, we have

$$
\left|J_{32}\right| \leq C\left(1+\varepsilon\left\|n_{R}^{\varepsilon}\right\|_{H^{s^{\prime}}}\right)\left(\left\|\mathbf{u}_{R}^{\varepsilon}\right\|_{H^{s^{\prime}}}+\left\|n_{R}^{\varepsilon}\right\|_{H^{s^{\prime}}}\right)
$$

thanks to 3.2.

From (3.84), by adding (3.86), 3.91), 3.92), 3.93), 3.94) and 3.95) together, we obtain 3.82). The proof is complete.

Proof of Proposition 3.1 By adding (3.27) and (3.82) together, we obtain

$$
\begin{aligned}
\frac{1}{2} \frac{d}{d t}\left\|\partial_{x}^{\alpha} \mathbf{u}_{R}^{\varepsilon}\right\|_{L^{2}}^{2} & +\frac{1}{2} \frac{d}{d t} \int \frac{1+\varepsilon \phi^{(1)}+\varepsilon^{2} \phi_{R}^{\varepsilon}}{n}\left|\partial_{x}^{\alpha} \phi_{R}^{\varepsilon}\right|^{2} \\
& +\frac{1}{2} \frac{d}{d t} \int \frac{\varepsilon}{n}\left|\bar{\nabla} \partial_{x}^{\alpha} \phi_{R}^{\varepsilon}\right|^{2}+\frac{1}{2} \frac{d}{d t} \int \frac{T_{i}}{n^{2}}\left|\partial_{x}^{\alpha} n_{R}^{\varepsilon}\right|^{2} d x \\
\leq & C C_{1}\left(C_{1}+\varepsilon\left\|\left(\mathbf{u}_{R}^{\varepsilon}, n_{R}^{\varepsilon}, \phi_{R}^{\varepsilon}\right)\right\|_{H^{s^{\prime}}}^{2}\right)\left\{1+\left\|\left(\mathbf{u}_{R}^{\varepsilon}, n_{R}^{\varepsilon}, \phi_{R}^{\varepsilon}\right)\right\|_{H^{s^{\prime}}}^{2}\right\}+I_{71}+J_{31},
\end{aligned}
$$

where $I_{71}$ and $J_{31}$ are given in (3.28) and (3.83) respectively. By integration by parts, we have

$$
\begin{aligned}
I_{71}+J_{31} & =-T_{i}\left\langle\frac{1}{\varepsilon n} \partial_{x}^{\alpha} \bar{\nabla} n_{R}^{\varepsilon}, \partial_{x}^{\alpha} \mathbf{u}_{R}^{\varepsilon}\right\rangle_{0}-T_{i}\left\langle\left(\bar{\nabla} \cdot \partial_{x}^{\alpha} \mathbf{u}_{R}^{\varepsilon}\right), \frac{1}{\varepsilon n} \partial_{x}^{\alpha} n_{R}^{\varepsilon}\right\rangle_{0} \\
& =T_{i}\left\langle\bar{\nabla}\left(\frac{1}{\varepsilon n}\right) \partial_{x}^{\alpha} n_{R}^{\varepsilon}, \partial_{x}^{\alpha} \mathbf{u}_{R}^{\varepsilon}\right\rangle_{0} \\
& \leq C\left(1+\varepsilon\left\|n_{R}^{\varepsilon}\right\|_{H^{3}}\right)\left\{\left\|n_{R}^{\varepsilon}\right\|_{H^{k}}+\left\|\mathbf{u}_{R}^{\varepsilon}\right\|_{H^{k}}\right\} .
\end{aligned}
$$

Inserting 3.977 into 3.96, we complete the proof of Proposition 3.1

Proof of Theorem 2.1 for $T_{i}>0$. Recalling (3.2), and integrating (3.26) over $[0, t]$ and taking summation over $|\alpha|=k$ and $0 \leq k \leq s^{\prime}$, we obtain

$$
\begin{aligned}
& \left\|\mathbf{u}_{R}^{\varepsilon}\right\|_{H^{s^{\prime}}}^{2}+\left\|\phi_{R}^{\varepsilon}\right\|_{H^{s^{\prime}}}^{2}+\varepsilon\left\|\bar{\nabla} \phi_{R}^{\varepsilon}\right\|_{H^{s^{\prime}}}^{2}+T_{i}\left\|n_{R}^{\varepsilon}\right\|_{H^{s^{\prime}}}^{2} \\
& \quad \leq C C_{\varepsilon}(0)+C C_{1} \int_{0}^{t}\left(C_{1}+\varepsilon\left\|\left(\mathbf{u}_{R}^{\varepsilon}, n_{R}^{\varepsilon}, \phi_{R}^{\varepsilon}\right)\right\|_{H^{s^{\prime}}}^{2}\right)\left\{1+\left\|\left(\mathbf{u}_{R}^{\varepsilon}, n_{R}^{\varepsilon}, \phi_{R}^{\varepsilon}\right)\right\|_{H^{s^{\prime}}}^{2}\right\} d r,
\end{aligned}
$$

where $C_{\varepsilon}(0)=\left\|\left(\mathbf{u}_{R}^{\varepsilon}, \phi_{R}^{\varepsilon}\right)(0)\right\|_{H^{s^{\prime}}}^{2}+\varepsilon\left\|\bar{\nabla} \phi_{R}^{\varepsilon}(0)\right\|_{H^{s^{\prime}}}^{2}+T_{i}\left\|n_{R}^{\varepsilon}(0)\right\|_{H^{s^{\prime}}}^{2}$. From 3.1), there exists some constant $0<\varepsilon_{0}<\varepsilon_{1}$ (in Lemma 3.1) such that $\varepsilon\left\|\left(\mathbf{u}_{R}^{\varepsilon}, n_{R}^{\varepsilon}, \phi_{R}^{\varepsilon}\right)\right\|_{H^{s^{\prime}}}^{2} \leq 1$ for any $0<\varepsilon<\varepsilon_{0}$. Since $C_{1}=$ $C_{1}\left(\sqrt{\varepsilon}\left\|n_{R}^{\varepsilon}\right\|_{H^{s^{\prime}}}\right)$ and is nondecreasing, we know that $C_{1} \leq C_{1}(1)$ when $0<\varepsilon<\varepsilon_{0}$. Since $T_{i}>0$, there exists some constant $C_{3}>1$ such that

$$
\left\|\mathbf{u}_{R}^{\varepsilon}\right\|_{H^{s^{\prime}}}^{2}+\left\|\phi_{R}^{\varepsilon}\right\|_{H^{s^{\prime}}}^{2}+\left\|n_{R}^{\varepsilon}\right\|_{H^{s^{\prime}}}^{2} \leq C_{3} C_{\varepsilon}(0)+C_{3} \int_{0}^{t}\left\{1+\left\|\left(\mathbf{u}_{R}^{\varepsilon}, n_{R}^{\varepsilon}, \phi_{R}^{\varepsilon}\right)\right\|_{H^{s^{\prime}}}^{2}\right\} d r .
$$

For any given $0<\tau_{0}<\tau_{*}$, let $C_{0}^{\prime}=\sup _{0<\varepsilon<1} C_{\varepsilon}(0)$ and $\tilde{C}$ in (3.1) satisfy $\tilde{C} \geq 2\left(1+C C_{0}^{\prime}\right) e^{C_{3} \tau_{0}}$, then by Gronwall inequality, we obtain

$$
\sup _{0 \leq t \leq \tau_{0}}\left\|\mathbf{u}_{R}^{\varepsilon}\right\|_{H^{s^{\prime}}}^{2}+\left\|\phi_{R}^{\varepsilon}\right\|_{H^{s^{\prime}}}^{2}+\left\|n_{R}^{\varepsilon}\right\|_{H^{s^{\prime}}}^{2} \leq\left(1+C C_{3}\right) e^{C_{3} \tau_{0}} \leq \tilde{C} .
$$

It is then standard to obtain uniform estimates for $\left\|\left(n_{R}^{\varepsilon}, \mathbf{u}_{R}^{\varepsilon}, \phi_{R}^{\varepsilon}\right)\right\|_{H^{s^{\prime}}}$ independent of $\varepsilon$ by the continuity method. The proof is complete for the case $T_{i}>0$. 


\section{Proof of Theorem 2.1 for $T_{i}=0$}

In this section, we prove Theorem 2.1 for the case of $T_{i}=0$ and $d=3$, i.e., we prove (2.33). In this case, $\bar{\nabla}$ and $\bar{\Delta}$ reduce to $\nabla=\left(\partial_{x_{1}}, \partial_{x_{2}}, \partial_{x_{3}}\right)$ and $\Delta=\partial_{x_{1}}^{2}+\partial_{x_{2}}^{2}+\partial_{x_{3}}^{2}$. Since $T_{i}=0$, we obtain $V=1$ from (B.6).

We also assume that (2.28) has smooth solutions in a small time $\tau_{\varepsilon}$ dependent on $\varepsilon$. As in Section 3, we let $\tilde{C}$ be a constant, which will be determined later, much larger than the bound of $\left\|\left(n_{R}^{\varepsilon}, \mathbf{u}_{R}^{\varepsilon}, \phi_{R}^{\varepsilon}\right)(0)\right\| \|_{s^{\prime}}$, such that on $\left[0, \tau_{\varepsilon}\right]$

$$
\sup _{\left[0, \tau_{\varepsilon}\right]}\left\|\left(n_{R}^{\varepsilon}, \mathbf{u}_{R}^{\varepsilon}, \phi_{R}^{\varepsilon}\right)\right\|_{s^{\prime}} \leq \tilde{C} .
$$

We will prove that $\tau_{\varepsilon}>\tau_{0}$ as $\varepsilon \rightarrow 0$ for some $0<\tau_{0}<\tau_{*}$, where $\tau_{*}$ is the existence time of the limit equation (B.13). Recalling the expressions for $n$ and $\mathbf{u}$ in (2.27), we immediately know that there exists some $\varepsilon_{1}=\varepsilon_{1}(\tilde{C})>0$ such that on $\left[0, \tau_{\varepsilon}\right]$,

$$
1 / 2<n<3 / 2, \quad|\mathbf{u}| \leq 1 / 2,
$$

for all $0<\varepsilon<\varepsilon_{1}$.

Proposition 4.1. Let $s^{\prime} \geq 4$ be an integer and $\left(n_{R}^{\varepsilon}, \boldsymbol{u}_{R}^{\varepsilon}, \phi_{R}^{\varepsilon}\right)$ be a solution to 2.28). Then for any integer $0 \leq k \leq s^{\prime}$, there holds

$$
\begin{gathered}
\frac{1}{2} \frac{d}{d t}\left\|\partial_{x}^{\alpha} \boldsymbol{u}_{R}^{\varepsilon}\right\|_{L^{2}}^{2}+\frac{1}{2} \frac{d}{d t} \int \frac{1+\varepsilon \phi^{(1)}+\varepsilon^{2} \phi_{R}^{\varepsilon}}{n}\left|\partial_{x}^{\alpha} \phi_{R}^{\varepsilon}\right|^{2}+\frac{1}{2} \frac{d}{d t} \int \frac{\varepsilon}{n}\left|\nabla \partial_{x}^{\alpha} \phi_{R}^{\varepsilon}\right|^{2} \\
\leq C C_{1}\left(1+\varepsilon\left\|\left(\boldsymbol{u}_{R}^{\varepsilon}, \phi_{R}^{\varepsilon}\right)\right\|_{s^{\prime}}^{2}\right)\left\{1+\left\|\left(\boldsymbol{u}_{R}^{\varepsilon}, \phi_{R}^{\varepsilon}\right)\right\|_{s^{\prime}}^{2}\right\},
\end{gathered}
$$

where $\alpha$ is any multi-index with $|\alpha|=k \leq s^{\prime}$ and $\left\|\left(\boldsymbol{u}_{R}^{\varepsilon}, \phi_{R}^{\varepsilon}\right)\right\|_{s^{\prime}}^{2}$ is defined in 2.31.

Proof. The proof of is basically contained in Section 3. Here, we only give the differences. Since $T_{i}=0$, there are no $I_{7}, I_{8}$ and $I_{9}$ in the proof of Lemma3.4 Then from Lemma3.4 we obtain

$$
\begin{gathered}
\frac{1}{2} \frac{d}{d t}\left\|\partial_{x}^{\alpha} \mathbf{u}_{R}^{\varepsilon}\right\|_{L^{2}}^{2}+\frac{1}{2} \frac{d}{d t} \int \frac{1+\varepsilon \phi^{(1)}+\varepsilon^{2} \phi_{R}^{\varepsilon}}{n}\left|\partial_{x}^{\alpha} \phi_{R}^{\varepsilon}\right|^{2}+\frac{1}{2} \frac{d}{d t} \int \frac{\varepsilon}{n}\left|\nabla \partial_{x}^{\alpha} \phi_{R}^{\varepsilon}\right|^{2} \\
\leq C C_{1}\left(C_{1}+\varepsilon\left\|\left(\mathbf{u}_{R}^{\varepsilon}, n_{R}^{\varepsilon}, \phi_{R}^{\varepsilon}\right)\right\|_{H^{s^{\prime}}}^{2}\right)\left\{1+\left\|\left(\mathbf{u}_{R}^{\varepsilon}, n_{R}^{\varepsilon}, \phi_{R}^{\varepsilon}\right)\right\|_{H^{s^{\prime}}}^{2}\right\} .
\end{gathered}
$$

The proof is then complete by replacing $\left\|n_{R}^{\varepsilon}\right\|_{H^{s^{\prime}}}^{2}$ with $\left\|\phi_{R}^{\varepsilon}\right\|_{s^{\prime}}$, thanks to Lemma 3.1

To obtain uniform estimates for the remainder term $\left(\mathbf{u}_{R}^{\varepsilon}, n_{R}^{\varepsilon}, \phi_{R}^{\varepsilon}\right)$, we will prove the following

Proposition 4.2. Let $\left(n_{R}^{\varepsilon}, \boldsymbol{u}_{R}^{\varepsilon}, \phi_{R}^{\varepsilon}\right)$ be a solution to (2.28) in $3 D$, then

$$
\begin{aligned}
\frac{\varepsilon}{2} \frac{d}{d t}\left\|\nabla \boldsymbol{u}_{R}^{\varepsilon}\right\|_{\dot{H}^{s^{\prime}}}^{2} & +\frac{1}{2} \frac{d}{d t}\left\langle\partial_{x}^{\alpha} \nabla \phi_{R}^{\varepsilon}, \frac{\varepsilon\left(1+\varepsilon \phi^{(1)}\right)}{n} \partial_{x}^{\alpha} \nabla \phi_{R}^{\varepsilon}\right\rangle_{0}+\frac{1}{2} \frac{d}{d t}\left\langle\partial_{x}^{\alpha} \Delta \phi_{R}^{\varepsilon}, \frac{\varepsilon^{2}}{n} \partial_{x}^{\alpha} \Delta \phi_{R}^{\varepsilon}\right\rangle_{0} \\
& \leq C\left(1+C_{1}\left(\sqrt{\varepsilon}\left\|\phi_{R}^{\varepsilon}\right\|_{H^{3}}\right)\right)\left(1+\varepsilon^{2}\left\|\left(\boldsymbol{u}_{R}^{\varepsilon}, \phi_{R}^{\varepsilon}\right)\right\|_{s^{\prime}}^{2}\right)\left(1+\left\|\left(\boldsymbol{u}_{R}^{\varepsilon}, \phi_{R}^{\varepsilon}\right)\right\|_{s^{\prime}}^{2}\right),
\end{aligned}
$$

where $\left\|\left(\boldsymbol{u}_{R}^{\varepsilon}, \phi_{R}^{\varepsilon}\right)\right\|_{s^{\prime}}^{2}$ is defined in (2.31) and $|\alpha|=s^{\prime}$ for any $s^{\prime} \geq 4$.

Proof. Let $\alpha$ be any multi-index with $|\alpha|=k \leq s^{\prime}$ for any $4 \leq s^{\prime} \leq s$. We take $\partial_{x}^{\alpha}$ of (2.28b) and then take inner product with $\varepsilon \Delta \partial_{x}^{\alpha} \mathbf{u}_{R}^{\varepsilon}$ in $L^{2}\left(\mathbb{R}^{3}\right)$. By integrating by parts, we obtain

$$
\begin{aligned}
\frac{\varepsilon}{2} \frac{d}{d t}\left\|\partial_{x}^{\alpha} \nabla \mathbf{u}_{R}^{\varepsilon}\right\|^{2}= & \left\langle\partial_{x_{1}} \partial_{x}^{\alpha} \nabla \mathbf{u}_{R}^{\varepsilon}, \partial_{x}^{\alpha} \nabla \mathbf{u}_{R}^{\varepsilon}\right\rangle_{0}-\left\langle\partial_{x}^{\alpha}\left(\nabla \mathbf{u} \cdot \nabla \mathbf{u}_{R}^{\varepsilon}\right), \partial_{x}^{\alpha} \nabla \mathbf{u}_{R}^{\varepsilon}\right\rangle_{0} \\
& -\left\langle\partial_{x}^{\alpha}\left(\mathbf{u} \cdot \nabla^{2} \mathbf{u}_{R}^{\varepsilon}\right), \partial_{x}^{\alpha} \nabla \mathbf{u}_{R}^{\varepsilon}\right\rangle_{0}-\varepsilon\left\langle\partial_{x}^{\alpha}\left(\nabla \mathbf{u}_{R}^{\varepsilon} \cdot \nabla \widetilde{\mathbf{u}}\right), \partial_{x}^{\alpha} \nabla \mathbf{u}_{R}^{\varepsilon}\right\rangle_{0} \\
& -\varepsilon\left\langle\partial_{x}^{\alpha}\left(\mathbf{u}_{R}^{\varepsilon} \cdot \nabla^{2} \widetilde{\mathbf{u}}\right), \partial_{x}^{\alpha} \nabla \mathbf{u}_{R}^{\varepsilon}\right\rangle_{0}-\varepsilon^{2}\left\langle\partial_{x}^{\alpha} \nabla \mathbf{R}_{\mathbf{u}}, \partial_{x}^{\alpha} \nabla \mathbf{u}_{R}^{\varepsilon}\right\rangle_{0} \\
& +\frac{1}{\varepsilon^{1 / 2}}\left\langle\mathbf{u}_{R}^{\varepsilon} \times \mathbf{e}_{1}, \mathbf{u}_{R}^{\varepsilon}\right\rangle_{\dot{H}^{k}}-\left\langle\partial_{x}^{\alpha} \Delta \phi_{R}^{\varepsilon}, \partial_{x}^{\alpha} \nabla \cdot \mathbf{u}_{R}^{\varepsilon}\right\rangle_{0} \\
= & K_{1}+\cdots+K_{8} .
\end{aligned}
$$


First, we know that $K_{1}$ and $K_{7}$ vanish by integration by parts.

Estimate of $K_{2}$. Using the commutator estimate, we have

$$
\begin{aligned}
\left|K_{2}\right| & \leq\left|\varepsilon\left\langle\partial_{x}^{\alpha}\left(\nabla \widetilde{\mathbf{u}} \cdot \nabla \mathbf{u}_{R}^{\varepsilon}\right), \partial_{x}^{\alpha} \nabla \mathbf{u}_{R}^{\varepsilon}\right\rangle_{0}\right|+\left|\varepsilon^{2}\left\langle\partial_{x}^{\alpha}\left(\nabla \mathbf{u}_{R}^{\varepsilon} \cdot \nabla \mathbf{u}_{R}^{\varepsilon}\right), \partial_{x}^{\alpha} \nabla \mathbf{u}_{R}^{\varepsilon}\right\rangle_{0}\right| \\
& \leq C \varepsilon\left\|\nabla \mathbf{u}_{R}^{\varepsilon}\right\|_{H^{k}}^{2}+\varepsilon^{2}\left\|\nabla \mathbf{u}_{R}^{\varepsilon}\right\|_{L^{\infty}}\left\|\nabla \mathbf{u}_{R}^{\varepsilon}\right\|_{H^{k}}^{2} \\
& \leq C\left(1+\varepsilon\left\|\nabla \mathbf{u}_{R}^{\varepsilon}\right\|_{H^{2}}\right)\left(\varepsilon\left\|\nabla \mathbf{u}_{R}^{\varepsilon}\right\|_{H^{k}}^{2}\right),
\end{aligned}
$$

where we have used Lemma C.1 and Sobolev embedding.

Estimate of $K_{3}$. By using commutator and integrating by parts, we have

$$
\begin{aligned}
K_{3} & =-\left\langle\mathbf{u} \cdot \partial_{x}^{\alpha} \nabla^{2} \mathbf{u}_{R}^{\varepsilon}, \partial_{x}^{\alpha} \nabla \mathbf{u}_{R}^{\varepsilon}\right\rangle_{0}-\left\langle\left[\partial_{x}^{\alpha}, \mathbf{u}\right] \cdot \nabla^{2} \mathbf{u}_{R}^{\varepsilon}, \partial_{x}^{\alpha} \nabla \mathbf{u}_{R}^{\varepsilon}\right\rangle_{0} \\
& =\frac{1}{2}\left\langle\nabla \cdot \mathbf{u} \partial_{x}^{\alpha} \nabla \mathbf{u}_{R}^{\varepsilon}, \partial_{x}^{\alpha} \nabla \mathbf{u}_{R}^{\varepsilon}\right\rangle_{0}-\left\langle\left[\partial_{x}^{\alpha}, \mathbf{u}\right] \cdot \nabla^{2} \mathbf{u}_{R}^{\varepsilon}, \partial_{x}^{\alpha} \nabla \mathbf{u}_{R}^{\varepsilon}\right\rangle_{0} \\
& =: K_{31}+K_{32} .
\end{aligned}
$$

Recalling (2.27), we know

$$
\begin{aligned}
K_{31} & \leq\left\langle\nabla \cdot \mathbf{u} \partial_{x}^{\alpha} \nabla \mathbf{u}_{R}^{\varepsilon}, \partial_{x}^{\alpha} \nabla \mathbf{u}_{R}^{\varepsilon}\right\rangle_{0} \\
& \leq C \varepsilon\left(1+\varepsilon\left\|\nabla \cdot \mathbf{u}_{R}^{\varepsilon}\right\|_{L^{\infty}}\right)\left\|\partial_{x}^{\alpha} \nabla \mathbf{u}_{R}^{\varepsilon}\right\|^{2} \\
& \leq C\left(1+\varepsilon\left\|\mathbf{u}_{R}^{\varepsilon}\right\|_{H^{3}}\right)\left(\varepsilon\left\|\nabla \mathbf{u}_{R}^{\varepsilon}\right\|_{H^{k}}^{2}\right) .
\end{aligned}
$$

Similarly, using (2.27) and Lemma C.1, we have

$$
\begin{aligned}
K_{32} \leq & C\left(\left\|\nabla^{2} \mathbf{u}_{R}^{\varepsilon}\right\|_{H^{k-1}}\|\mathbf{u}\|_{L^{\infty}}+\left\|\nabla^{2} \mathbf{u}_{R}^{\varepsilon}\right\|_{L^{\infty}}\|\mathbf{u}\|_{H^{k}}\right)\left\|\partial_{x}^{\alpha} \nabla \mathbf{u}_{R}^{\varepsilon}\right\|^{2} \\
\leq & C\left(1+\varepsilon\left\|\mathbf{u}_{R}^{\varepsilon}\right\|_{L^{\infty}}\right)\left(\varepsilon\left\|\nabla \mathbf{u}_{R}^{\varepsilon}\right\|_{H^{k}}^{2}\right) \\
& +C \varepsilon\left\|\nabla^{2} \mathbf{u}_{R}^{\varepsilon}\right\|_{L^{\infty}}\left(1+\varepsilon\left\|\mathbf{u}_{R}^{\varepsilon}\right\|_{H^{k}}\right)\left\|\partial_{x}^{\alpha} \nabla \mathbf{u}_{R}^{\varepsilon}\right\|_{L^{2}} \\
\leq & C\left(1+\varepsilon\left\|\mathbf{u}_{R}^{\varepsilon}\right\|_{H^{4}}\right)\left(\left\|\mathbf{u}_{R}^{\varepsilon}\right\|_{H^{s^{\prime}}}^{2}+\varepsilon\left\|\nabla \mathbf{u}_{R}^{\varepsilon}\right\|_{H^{s^{\prime}}}^{2}\right),
\end{aligned}
$$

where $k \leq s^{\prime}$ and $s^{\prime} \geq 4$. Therefore, using 2.31, we have

$$
K_{3} \leq C\left(1+\varepsilon\left\|\mathbf{u}_{R}^{\varepsilon}\right\|_{H^{4}}\right)\left\|\mathbf{u}_{R}^{\varepsilon}\right\|_{S^{\prime}}^{2} .
$$

Estimate of $K_{4}, K_{5}$ and $K_{6}$. The estimate of $K_{4}$ and $K_{5}$ is similar to $K_{2}$ and $K_{3}$. We obtain

$$
K_{4}, K_{5} \leq C\left\|\mathbf{u}_{R}^{\varepsilon}\right\|_{s^{\prime}}^{2} .
$$

Since $\mathbf{R}_{\mathbf{u}}$ depends only on $\widetilde{\mathbf{u}}$, we know that

$$
K_{6} \leq C\left(1+\varepsilon\left\|\nabla \mathbf{u}_{R}^{\varepsilon}\right\|_{H^{s^{\prime}}}^{2}\right)
$$

Summarizing, we have

$$
\sum_{i=1}^{7}\left|K_{i}\right| \leq C\left(1+\varepsilon\left\|\mathbf{u}_{R}^{\varepsilon}\right\|_{H^{4}}\right)\left(1+\left\|\mathbf{u}_{R}^{\varepsilon}\right\|_{s^{\prime}}^{2}\right)
$$

Estimate of $K_{8}$. Taking $\partial_{x}^{\alpha}$ of (2.28a with $\bar{\nabla}=\nabla$ in 3D, we obtain

$$
\begin{aligned}
\frac{1}{\varepsilon} \nabla \cdot \partial_{x}^{\alpha} \mathbf{u}_{R}^{\varepsilon}= & \frac{1}{n}\left\{\frac{V \mathbf{e}_{1}-\mathbf{u}}{\varepsilon} \cdot \nabla \partial_{x}^{\alpha} n_{R}^{\varepsilon}-\partial_{t} \partial_{x}^{\alpha} n_{R}^{\varepsilon}-\left[\partial_{x}^{\alpha}, \frac{\mathbf{u}}{\varepsilon}\right] \cdot \nabla n_{R}^{\varepsilon}\right. \\
& \left.-\left[\partial_{x}^{\alpha}, \frac{n}{\varepsilon}\right] \nabla \cdot \mathbf{u}_{R}^{\varepsilon}-\partial_{x}^{\alpha}\left(n_{R}^{\varepsilon} \nabla \cdot \widetilde{\mathbf{u}}+\mathbf{u}_{R}^{\varepsilon} \cdot \nabla \widetilde{n}+\varepsilon R_{n}\right)\right\}
\end{aligned}
$$


where $|\alpha|=k$. Accordingly, we have the decomposition

$$
\begin{aligned}
K_{8}= & -\left\langle\partial_{x}^{\alpha} \Delta \phi_{R}^{\varepsilon}, \frac{V \mathbf{e}_{1}-\mathbf{u}}{n} \cdot \nabla \partial_{x}^{\alpha} n_{R}^{\varepsilon}\right\rangle_{0}+\left\langle\partial_{x}^{\alpha} \Delta \phi_{R}^{\varepsilon}, \frac{\varepsilon}{n} \partial_{t} \partial_{x}^{\alpha} n_{R}^{\varepsilon}\right\rangle_{0} \\
& +\left\langle\partial_{x}^{\alpha} \Delta \phi_{R}^{\varepsilon}, \frac{1}{n}\left[\partial_{x}^{\alpha}, \mathbf{u}\right] \cdot \nabla n_{R}^{\varepsilon}\right\rangle_{0}+\left\langle\partial_{x}^{\alpha} \Delta \phi_{R}^{\varepsilon}, \frac{1}{n}\left[\partial_{x}^{\alpha}, n\right] \nabla \cdot \mathbf{u}_{R}^{\varepsilon}\right\rangle_{0} \\
& +\left\langle\partial_{x}^{\alpha} \Delta \phi_{R}^{\varepsilon}, \frac{\varepsilon}{n} \partial_{x}^{\alpha}\left(n_{R}^{\varepsilon} \nabla \cdot \widetilde{\mathbf{u}}+\mathbf{u}_{R}^{\varepsilon} \cdot \nabla \widetilde{n}+\varepsilon R_{n}\right)\right\rangle_{0} \\
= & : K_{81}+\cdots+K_{85} .
\end{aligned}
$$

Recalling $\mathbf{u}=\varepsilon \widetilde{\mathbf{u}}+\varepsilon^{2} \mathbf{u}_{R}^{\varepsilon}$ in 2.27, we have

$$
\begin{aligned}
\left|K_{83}\right| & \leq\left|\left\langle\partial_{x}^{\alpha} \Delta \phi_{R}^{\varepsilon}, \frac{1}{n}\left[\partial_{x}^{\alpha}, \mathbf{u}\right] \nabla n_{R}^{\varepsilon}\right\rangle_{0}\right| \\
& \leq C\left\|\partial_{x}^{\alpha} \Delta \phi_{R}^{\varepsilon}\right\|_{L^{2}}^{2}\left\{\left\|\nabla n_{R}^{\varepsilon}\right\|_{H^{k-1}}\|\nabla \mathbf{u}\|_{L^{\infty}}+\left\|\nabla n_{R}^{\varepsilon}\right\|\left\|_{L^{\infty}}\right\| \nabla \mathbf{u} \|_{H^{k-1}}\right\} \\
& \leq C \varepsilon\left\|\partial_{x}^{\alpha} \Delta \phi_{R}^{\varepsilon}\right\|_{L^{2}}\left\{\left(1+\varepsilon\left\|\nabla \mathbf{u}_{R}^{\varepsilon}\right\|_{L^{\infty}}\right)\left\|n_{R}^{\varepsilon}\right\|_{H^{3}}+\left(1+\varepsilon\left\|\mathbf{u}_{R}^{\varepsilon}\right\|_{H^{k}}\right)\left\|n_{R}^{\varepsilon}\right\|_{H^{k}}\right\} \\
& \leq C \varepsilon^{2}\left\|\Delta \phi_{R}^{\varepsilon}\right\|_{H^{s^{\prime}}}^{2}+C\left(1+\varepsilon^{2}\left\|\mathbf{u}_{R}^{\varepsilon}\right\|_{H^{s^{\prime}}}^{2}\left\|n_{R}^{\varepsilon}\right\|_{H^{s^{\prime}}}^{2},\right.
\end{aligned}
$$

where $k \leq s^{\prime}$ and $s^{\prime} \geq 4$. Here, we have used the fact that $1 / 2 \leq n \leq 3 / 2$ is bounded from above and below by 4.1 .

The estimate for $K_{84}$ and $K_{85}$ are similar to $K_{83}$. From (3.43) and (3.45), we know

$$
\left|K_{84}\right|+\left|K_{85}\right| \leq C \varepsilon^{2}\left\|\Delta \phi_{R}^{\varepsilon}\right\|_{H^{s^{\prime}}}^{2}+C\left(1+\varepsilon^{2}\left\|\mathbf{u}_{R}^{\varepsilon}\right\|_{H^{s^{s}}}^{2}\right)\left\|\left(n_{R}^{\varepsilon}, \mathbf{u}_{R}^{\varepsilon}, \phi_{R}^{\varepsilon}\right)\right\|_{H^{s^{s}}}^{2}
$$

where $s^{\prime} \geq 4$.

Adding (4.17) and (4.18) together and using Lemma 3.1, we have

$$
\left|K_{83}\right|+\left|K_{84}\right|+\left|K_{85}\right| \leq C\left(1+\varepsilon^{2}\left\|\mathbf{u}_{R}^{\varepsilon}\right\|_{H^{s^{\prime}}}^{2}\right)\left\|\left(\mathbf{u}_{R}^{\varepsilon}, \phi_{R}^{\varepsilon}\right)\right\|_{s^{\prime}}^{2},
$$

where $\left\|\left(\mathbf{u}_{R}^{\varepsilon}, \phi_{R}^{\varepsilon}\right)\right\|_{s^{\prime}}^{2}$ is defined in 2.31).

The proof of Proposition 4.2 is complete once $K_{81}$ and $K_{82}$ are estimated in the following Lemma 4.1 and Lemma 4.2

Lemma 4.1. Let $\left(n_{R}^{\varepsilon}, \boldsymbol{u}_{R}^{\varepsilon}, \phi_{R}^{\varepsilon}\right)$ be a solution to (2.28), then

$$
\left|K_{81}\right| \leq C_{1}\left(\sqrt{\varepsilon}\left\|\phi_{R}^{\varepsilon}\right\|_{H^{3}}\right)\left(1+\varepsilon^{2}\left\|\left(\boldsymbol{u}_{R}^{\varepsilon}, \phi_{R}^{\varepsilon}\right)\right\| \|_{s^{\prime}}^{2}\right)\left\|\phi_{R}^{\varepsilon}\right\|_{s^{\prime}}^{2},
$$

where $\left\|\left(\boldsymbol{u}_{R}^{\varepsilon}, \phi_{R}^{\varepsilon}\right)\right\|_{s^{\prime}}^{2}$ is given in (2.31) and $K_{81}$ is given in (4.16).

Proof. Let $\alpha$ be the multi-index in Proposition 4.2 . Taking $\partial_{x}^{\alpha}$ of $2.28 \mathrm{c}$, we have

$$
\partial_{x}^{\alpha} n_{R}^{\varepsilon}=\partial_{x}^{\alpha} \phi_{R}^{\varepsilon}-\varepsilon \Delta \partial_{x}^{\alpha} \phi_{R}^{\varepsilon}+\varepsilon \partial_{x}^{\alpha}\left(\phi^{(1)} \phi_{R}^{\varepsilon}\right)+\varepsilon^{3 / 2} \partial_{x}^{\alpha} R_{\phi} .
$$

$K_{81}$ in (4.16) is then divided into

$$
\begin{aligned}
K_{81}= & -\left\langle\partial_{x}^{\alpha} \Delta \phi_{R}^{\varepsilon}, \frac{\mathbf{e}_{1}-\mathbf{u}}{n} \cdot \nabla \partial_{x}^{\alpha} n_{R}^{\varepsilon}\right\rangle_{0} \\
= & -\left\langle\partial_{x}^{\alpha} \Delta \phi_{R}^{\varepsilon}, \frac{\mathbf{e}_{1}-\mathbf{u}}{n} \cdot \nabla \partial_{x}^{\alpha} \phi_{R}^{\varepsilon}\right\rangle_{0}+\varepsilon\left\langle\partial_{x}^{\alpha} \Delta \phi_{R}^{\varepsilon}, \frac{\mathbf{e}_{1}-\mathbf{u}}{n} \cdot \nabla \Delta \partial_{x}^{\alpha} \phi_{R}^{\varepsilon}\right\rangle_{0} \\
& -\varepsilon\left\langle\partial_{x}^{\alpha} \Delta \phi_{R}^{\varepsilon}, \frac{\mathbf{e}_{1}-\mathbf{u}}{n} \cdot \nabla \partial_{x}^{\alpha}\left(\phi^{(1)} \phi_{R}^{\varepsilon}\right)\right\rangle_{0}-\varepsilon^{3 / 2}\left\langle\partial_{x}^{\alpha} \Delta \phi_{R}^{\varepsilon}, \frac{\mathbf{e}_{1}-\mathbf{u}}{n} \cdot \nabla \partial_{x}^{\alpha} R_{\phi}\right\rangle_{0} \\
= & : K_{811}+K_{812}+K_{813}+K_{814},
\end{aligned}
$$


where $|\alpha|=k \leq s^{\prime}$ for $s^{\prime} \geq 3$.

Estimate of $K_{811}$. By integrating by parts twice, we have

$$
\begin{aligned}
K_{811} & =-\left\langle\partial_{x}^{\alpha} \nabla \cdot \nabla \phi_{R}^{\varepsilon}, \frac{\mathbf{e}_{1}-\mathbf{u}}{n} \partial_{x}^{\alpha} \nabla \phi_{R}^{\varepsilon}\right\rangle_{0} \\
& =\left\langle\partial_{x}^{\alpha} \nabla \phi_{R}^{\varepsilon}, \frac{\mathbf{e}_{1}-\mathbf{u}}{n} \partial_{x}^{\alpha} \nabla^{2} \phi_{R}^{\varepsilon}\right\rangle_{0}+\left\langle\partial_{x}^{\alpha} \nabla \phi_{R}^{\varepsilon}, \nabla\left(\frac{\mathbf{e}_{1}-\mathbf{u}}{n}\right) \partial_{x}^{\alpha} \nabla \phi_{R}^{\varepsilon}\right\rangle_{0} \\
& =-\frac{1}{2}\left\langle\partial_{x}^{\alpha} \nabla \phi_{R}^{\varepsilon}, \nabla \cdot\left(\frac{\mathbf{e}_{1}-\mathbf{u}}{n}\right) \partial_{x}^{\alpha} \nabla \phi_{R}^{\varepsilon}\right\rangle_{0}+\left\langle\partial_{x}^{\alpha} \nabla \phi_{R}^{\varepsilon}, \nabla\left(\frac{\mathbf{e}_{1}-\mathbf{u}}{n}\right) \partial_{x}^{\alpha} \nabla \phi_{R}^{\varepsilon}\right\rangle_{0}
\end{aligned}
$$

Recalling 2.27, we have

$$
\left\|\nabla\left(\frac{\mathbf{e}_{1}-\mathbf{u}}{n}\right)\right\|_{L^{\infty}},\left\|\nabla \cdot\left(\frac{\mathbf{e}_{1}-\mathbf{u}}{n}\right)\right\|_{L^{\infty}} \leq C \varepsilon\left(1+\varepsilon\left\|\nabla n_{R}^{\varepsilon}\right\|_{L^{\infty}}+\varepsilon\left\|\nabla \mathbf{u}_{R}^{\varepsilon}\right\|_{L^{\infty}}\right) .
$$

Therefore, using Lemma3.1, we obtain

$$
\begin{aligned}
\left|K_{811}\right| & \leq C \varepsilon\left(1+\varepsilon\left(\left\|n_{R}^{\varepsilon}\right\|_{H^{3}}+\left\|\mathbf{u}_{R}^{\varepsilon}\right\|_{H^{3}}\right)\right)\left\|\bar{\nabla} \partial_{x}^{\alpha} \phi_{R}^{\varepsilon}\right\|^{2} \\
& \leq C\left(1+\varepsilon\left\|\left(\mathbf{u}_{R}^{\varepsilon}, \phi_{R}^{\varepsilon}\right)\right\| \|_{s^{\prime}}\right)\left\|\phi_{R}^{\varepsilon}\right\|_{s^{\prime}}^{2},
\end{aligned}
$$

where $s^{\prime} \geq 3$.

Estimate of $K_{812}$. By integrating by parts, we obtain

$$
K_{812}=-\frac{\varepsilon}{2}\left\langle\partial_{x}^{\alpha} \Delta \phi_{R}^{\varepsilon}, \nabla \cdot \frac{\mathbf{e}_{1}-\mathbf{u}}{n} \Delta \partial_{x}^{\alpha} \phi_{R}^{\varepsilon}\right\rangle_{0} .
$$

Using 4.21, we obtain

$$
\begin{aligned}
\left|K_{812}\right| & \leq C \varepsilon^{2}\left(1+\varepsilon\left(\left\|\left(n_{R}^{\varepsilon}, \mathbf{u}_{R}^{\varepsilon}\right)\right\|_{H^{3}}\right)\right)\left\|\Delta \partial_{x}^{\alpha} \phi_{R}^{\varepsilon}\right\|^{2} \\
& \leq C\left(1+\varepsilon\left\|\left(\mathbf{u}_{R}^{\varepsilon}, \phi_{R}^{\varepsilon}\right)\right\|_{s^{\prime}}\right)\left\|\phi_{R}^{\varepsilon}\right\|_{s^{\prime}}^{2},
\end{aligned}
$$

thanks to Lemma3.1, where $s^{\prime} \geq 3$.

Estimate of $K_{813}$. By using the commutator and integrating by parts twice, we have

$$
\begin{aligned}
K_{813}= & -\varepsilon\left\langle\partial_{x}^{\alpha} \Delta \phi_{R}^{\varepsilon}, \frac{\left(\mathbf{e}_{1}-\mathbf{u}\right) \phi^{(1)}}{n} \cdot \nabla \partial_{x}^{\alpha} \phi_{R}^{\varepsilon}\right\rangle_{0}-\varepsilon\left\langle\partial_{x}^{\alpha} \Delta \phi_{R}^{\varepsilon}, \frac{\left(\mathbf{e}_{1}-\mathbf{u}\right)}{n} \cdot\left[\nabla \partial_{x}^{\alpha}, \phi^{(1)}\right] \phi_{R}^{\varepsilon}\right\rangle_{0} \\
= & -\frac{\varepsilon}{2}\left\langle\partial_{x}^{\alpha} \nabla \phi_{R}^{\varepsilon}, \nabla \cdot\left(\frac{\left(\mathbf{e}_{1}-\mathbf{u}\right) \phi^{(1)}}{n}\right) \nabla \partial_{x}^{\alpha} \phi_{R}^{\varepsilon}\right\rangle_{0}+\varepsilon\left\langle\partial_{x}^{\alpha} \nabla \phi_{R}^{\varepsilon}, \nabla\left(\frac{\left(\mathbf{e}_{1}-\mathbf{u}\right) \phi^{(1)}}{n}\right) \nabla \partial_{x}^{\alpha} \phi_{R}^{\varepsilon}\right\rangle_{0} \\
& -\varepsilon\left\langle\partial_{x}^{\alpha} \Delta \phi_{R}^{\varepsilon}, \frac{\left(\mathbf{e}_{1}-\mathbf{u}\right)}{n} \cdot\left[\nabla \partial_{x}^{\alpha}, \phi^{(1)}\right] \phi_{R}^{\varepsilon}\right\rangle_{0} \\
= & : K_{8131}+K_{8132}+K_{813} .
\end{aligned}
$$

Since

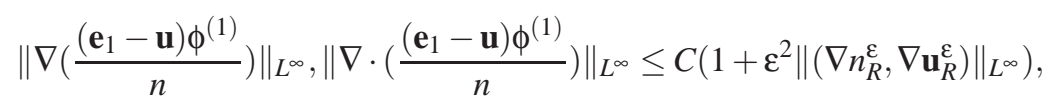

we obtain

$$
\begin{aligned}
\left|K_{8131}\right|+\left|K_{8132}\right| & \leq C \varepsilon\left(1+\varepsilon^{2}\left(\left\|n_{R}^{\varepsilon}\right\|_{H^{3}}+\left\|\mathbf{u}_{R}^{\varepsilon}\right\|_{H^{3}}\right)\right)\left\|\nabla \partial_{x}^{\alpha} \phi_{R}^{\varepsilon}\right\|^{2} \\
& \leq C\left(1+\varepsilon\left\|\left(\mathbf{u}_{R}^{\varepsilon}, \phi_{R}^{\varepsilon}\right)\right\| \|_{s^{\prime}}\right)\left\|\phi_{R}^{\varepsilon}\right\|_{s^{\prime}}^{2}
\end{aligned}
$$

From 4.2], we know that

$$
\begin{aligned}
\left|K_{8133}\right| & \leq C \varepsilon^{2}\left\|\Delta \partial_{x}^{\alpha} \phi_{R}^{\varepsilon}\right\|^{2}+C\left\|\left[\nabla \partial_{x}^{\alpha}, \phi^{(1)}\right] \phi_{R}^{\varepsilon}\right\|_{L^{2}}^{2} \\
& \leq C \varepsilon^{2}\left\|\Delta \partial_{x}^{\alpha} \phi_{R}^{\varepsilon}\right\|^{2}+C\left\|\phi_{R}^{\varepsilon}\right\|_{H^{s^{\prime}}}^{2}
\end{aligned}
$$


where we have used 3.59 . Therefore,

$$
\left|K_{813}\right| \leq C\left(1+\varepsilon\|\|\left(\mathbf{u}_{R}^{\varepsilon}, \phi_{R}^{\varepsilon}\right)\|\|_{s^{\prime}}\right)\left\|\phi_{R}^{\varepsilon}\right\|_{s^{\prime}}^{2} .
$$

Estimate of $K_{814}$. Using (2.22) in Lemma2.1, we have

$$
\begin{aligned}
\left|K_{814}\right| & \leq C \varepsilon^{3 / 2}\left\|\Delta \partial_{x}^{\alpha} \phi_{R}^{\varepsilon}\right\|\left\|\partial_{x}^{\alpha} \nabla R_{\phi}\right\| \\
& \leq C \varepsilon^{2}\left\|\Delta \phi_{R}^{\varepsilon}\right\|_{H^{s^{\prime}}}^{2}+\varepsilon C_{1}\left(\sqrt{\varepsilon}\left\|\phi_{R}^{\varepsilon}\right\|_{H^{s^{\prime}}}\right)\left\|\nabla \phi_{R}^{\varepsilon}\right\|_{H^{s^{\prime}}}^{2},
\end{aligned}
$$

where we have used $|\alpha|=k \leq s^{\prime}$ for $s^{\prime} \geq 3$.

Adding (4.22)-4.29) together, we have

$$
\left|K_{81}\right| \leq C_{1}\left(\sqrt{\varepsilon}\left\|\phi_{R}^{\varepsilon}\right\|_{H^{3}}\right)\left(1+\varepsilon^{2}\left\|\left(\mathbf{u}_{R}^{\varepsilon}, \phi_{R}^{\varepsilon}\right)\right\|_{s^{\prime}}^{2}\right)\left\|\phi_{R}^{\varepsilon}\right\|_{H^{s^{\prime}}}^{2},
$$

where $\left\|\left(\mathbf{u}_{R}^{\varepsilon}, \phi_{R}^{\varepsilon}\right)\right\|_{s^{\prime}}^{2}$ is given in 2.31). The proof of Lemma4.1 is complete.

Lemma 4.2. Let $\left(n_{R}^{\varepsilon}, \boldsymbol{u}_{R}^{\varepsilon}, \phi_{R}^{\varepsilon}\right)$ be a solution to (2.28) in $3 D$, then

$$
\begin{aligned}
K_{821} \leq & -\frac{1}{2} \frac{d}{d t}\left\langle\partial_{x}^{\alpha} \nabla \phi_{R}^{\varepsilon}, \frac{\varepsilon}{n} \partial_{x}^{\alpha} \nabla \phi_{R}^{\varepsilon}\right\rangle_{0}-\frac{1}{2} \frac{d}{d t}\left\langle\partial_{x}^{\alpha} \Delta \phi_{R}^{\varepsilon}, \frac{\varepsilon^{2}}{n} \partial_{x}^{\alpha} \Delta \phi_{R}^{\varepsilon}\right\rangle_{0} \\
& -\frac{1}{2} \frac{d}{d t}\left\langle\partial_{x}^{\alpha} \nabla \phi_{R}^{\varepsilon},\left(\frac{\varepsilon^{2} \phi^{(1)}}{n}\right) \partial_{x}^{\alpha} \nabla \phi_{R}^{\varepsilon}\right\rangle_{0}-\frac{1}{2} \frac{d}{d t}\left\langle\partial_{x}^{\alpha} \nabla \phi_{R}^{\varepsilon}, \frac{\varepsilon^{3} \phi_{R}^{\varepsilon}}{n} \partial_{x}^{\alpha} \nabla \phi_{R}^{\varepsilon}\right\rangle_{0} \\
& +C\left(C_{1}(\sqrt{\varepsilon} \tilde{C})+\varepsilon^{2}\left\|\left(\boldsymbol{u}_{R}^{\varepsilon}, \phi_{R}^{\varepsilon}\right)\right\|_{3}^{2}\right)\left\{1+\left\|\left(\boldsymbol{u}_{R}^{\varepsilon}, \phi_{R}^{\varepsilon}\right)\right\|_{s^{\prime}}^{2}\right\},
\end{aligned}
$$

where $|\alpha|=s^{\prime}$ for any $s^{\prime} \geq 4, K_{82}$ is given in (4.16) and $\left\|\left(\boldsymbol{u}_{R}^{\varepsilon}, \phi_{R}^{\varepsilon}\right)\right\|_{s^{\prime}}^{2}$ is defined in (2.31).

Proof. Recall that $\bar{\nabla}=\nabla, \bar{\Delta}=\Delta$ in 3D and $K_{82}$ is defined in (4.16)

$$
K_{82}=\left\langle\partial_{x}^{\alpha} \Delta \phi_{R}^{\varepsilon}, \frac{\varepsilon}{n} \partial_{t} \partial_{x}^{\alpha} n_{R}^{\varepsilon}\right\rangle_{0}
$$

Taking $\partial_{x}^{\alpha}$ of $2.28 \mathrm{c}$, we have

$$
\partial_{x}^{\alpha} n_{R}^{\varepsilon}=\partial_{x}^{\alpha} \phi_{R}^{\varepsilon}-\varepsilon \Delta \partial_{x}^{\alpha} \phi_{R}^{\varepsilon}+\varepsilon \partial_{x}^{\alpha}\left(\phi^{(1)} \phi_{R}^{\varepsilon}\right)+\frac{\varepsilon^{2}}{2} \partial_{x}^{\alpha}\left[\left(\phi_{R}^{\varepsilon}\right)^{2}\right]+\varepsilon^{2} \partial_{x}^{\alpha} \bar{R}_{\phi} .
$$

Inserting this into $K_{82}$, we have

$$
\begin{aligned}
K_{82}= & \left\langle\partial_{x}^{\alpha} \Delta \phi_{R}^{\varepsilon}, \frac{\varepsilon}{n} \partial_{t} \partial_{x}^{\alpha} \phi_{R}^{\varepsilon}\right\rangle_{0}-\varepsilon\left\langle\partial_{x}^{\alpha} \Delta \phi_{R}^{\varepsilon}, \frac{\varepsilon}{n} \partial_{t} \Delta \partial_{x}^{\alpha} \phi_{R}^{\varepsilon}\right\rangle_{0} \\
& +\varepsilon\left\langle\partial_{x}^{\alpha} \Delta \phi_{R}^{\varepsilon}, \frac{\varepsilon}{n} \partial_{t} \partial_{x}^{\alpha}\left(\phi^{(1)} \phi_{R}^{\varepsilon}\right)\right\rangle_{0}+\frac{\varepsilon^{2}}{2}\left\langle\partial_{x}^{\alpha} \Delta \phi_{R}^{\varepsilon}, \frac{\varepsilon}{n} \partial_{t} \partial_{x}^{\alpha}\left[\left(\phi_{R}^{\varepsilon}\right)^{2}\right]\right\rangle_{0} \\
& +\varepsilon^{2}\left\langle\partial_{x}^{\alpha} \Delta \phi_{R}^{\varepsilon}, \frac{\varepsilon}{n} \partial_{t} \partial_{x}^{\alpha} \bar{R}_{\phi}\right\rangle_{0} \\
= & : K_{821}+K_{822}+K_{823}+K_{824}+K_{825}
\end{aligned}
$$

Estimate of $K_{821}$. By integration by parts, we obtain

$$
\begin{aligned}
K_{821} & =-\left\langle\partial_{x}^{\alpha} \nabla \phi_{R}^{\varepsilon}, \frac{\varepsilon}{n} \partial_{t} \partial_{x}^{\alpha} \nabla \phi_{R}^{\varepsilon}\right\rangle_{0}-\left\langle\partial_{x}^{\alpha} \nabla \phi_{R}^{\varepsilon}, \nabla\left(\frac{\varepsilon}{n}\right) \partial_{t} \partial_{x}^{\alpha} \phi_{R}^{\varepsilon}\right\rangle_{0} \\
& =: K_{8211}+K_{8212} .
\end{aligned}
$$

By integrating in time, we obtain

$$
K_{8211}=-\frac{1}{2} \frac{d}{d t}\left\langle\partial_{x}^{\alpha} \nabla \phi_{R}^{\varepsilon}, \frac{\varepsilon}{n} \partial_{x}^{\alpha} \nabla \phi_{R}^{\varepsilon}\right\rangle_{0}+\frac{1}{2}\left\langle\partial_{x}^{\alpha} \nabla \phi_{R}^{\varepsilon}, \partial_{t}\left(\frac{\varepsilon}{n}\right) \partial_{x}^{\alpha} \nabla \phi_{R}^{\varepsilon}\right\rangle_{0} .
$$


From (3.65), we then have

$$
\begin{aligned}
K_{8211} & \leq-\frac{1}{2} \frac{d}{d t}\left\langle\partial_{x}^{\alpha} \nabla \phi_{R}^{\varepsilon}, \frac{\varepsilon}{n} \partial_{x}^{\alpha} \nabla \phi_{R}^{\varepsilon}\right\rangle_{0}+C\left(1+\varepsilon^{2}\left\|\partial_{t} n_{R}^{\varepsilon}\right\|_{L^{\infty}}\right)\left(\varepsilon\left\|\partial_{x}^{\alpha} \nabla \phi_{R}^{\varepsilon}\right\|^{2}\right) \\
& \leq-\frac{1}{2} \frac{d}{d t}\left\langle\partial_{x}^{\alpha} \nabla \phi_{R}^{\varepsilon}, \frac{\varepsilon}{n} \partial_{x}^{\alpha} \nabla \phi_{R}^{\varepsilon}\right\rangle_{0}+C\left(1+\varepsilon\left\|\left(\mathbf{u}_{R}^{\varepsilon}, \phi_{R}^{\varepsilon}\right)\right\| \|_{3}\right)\left\|\phi_{R}^{\varepsilon}\right\|_{s^{\prime}}^{2}
\end{aligned}
$$

where $\|\cdot \cdot\|_{s^{\prime}}$ is defined in (2.31) and we have used (3.12) in Corollary 3.2.

Recalling 2.27, we have

$$
\left\|\nabla\left(\frac{\varepsilon}{n}\right)\right\|_{L^{\infty}} \leq C \varepsilon^{2}\left(1+\varepsilon\left\|\nabla n_{R}^{\varepsilon}\right\|_{L^{\infty}}\right) .
$$

It the follows from (4.34) that

$$
\begin{aligned}
K_{8212} & \leq C \varepsilon^{2}\left(1+\varepsilon\left\|\nabla n_{R}^{\varepsilon}\right\|_{L^{\infty}}\right)\left\|\partial_{x}^{\alpha} \nabla \phi_{R}^{\varepsilon}\right\|_{L^{2}}\left\|\partial_{t} \partial_{x}^{\alpha} \phi_{R}^{\varepsilon}\right\|_{L^{2}} \\
& \leq C\left(1+\varepsilon\left\|n_{R}^{\varepsilon}\right\|_{H^{3}}\right)\left\{\varepsilon\left\|\nabla \phi_{R}^{\varepsilon}\right\|_{H^{k}}^{2}+\varepsilon\left\|\varepsilon \partial_{t} \nabla \phi_{R}^{\varepsilon}\right\|_{H^{k-1}}^{2}\right\} \\
& \leq C\left(1+\varepsilon\left\|\phi_{R}^{\varepsilon}\right\|_{3}\right)\left\|\left(\mathbf{u}_{R}^{\varepsilon}, \phi_{R}^{\varepsilon}\right)\right\|_{s^{\prime}}^{2}
\end{aligned}
$$

where we have used Lemma3.1 Corollary 3.1 and $k \leq s^{\prime}$.

Therefore, by adding (4.36) and 4.38, we obtain

$$
\begin{aligned}
K_{821} & \leq-\frac{1}{2} \frac{d}{d t}\left\langle\partial_{x}^{\alpha} \nabla \phi_{R}^{\varepsilon}, \frac{\varepsilon}{n} \partial_{x}^{\alpha} \nabla \phi_{R}^{\varepsilon}\right\rangle_{0}+C\left(1+\varepsilon^{2}\left\|\partial_{t} n_{R}^{\varepsilon}\right\|_{L^{\infty}}\right)\left(\varepsilon\left\|\partial_{x}^{\alpha} \nabla \phi_{R}^{\varepsilon}\right\|^{2}\right) \\
& \leq-\frac{1}{2} \frac{d}{d t}\left\langle\partial_{x}^{\alpha} \nabla \phi_{R}^{\varepsilon}, \frac{\varepsilon}{n} \partial_{x}^{\alpha} \nabla \phi_{R}^{\varepsilon}\right\rangle_{0}+C\left(1+\varepsilon\left\|\left(\mathbf{u}_{R}^{\varepsilon}, \phi_{R}^{\varepsilon}\right)\right\| \|_{3}\right)\left\|\left(\mathbf{u}_{R}^{\varepsilon}, \phi_{R}^{\varepsilon}\right)\right\|_{s^{\prime}}^{2}
\end{aligned}
$$

Estimate of $K_{822}$. Recall that in 4.33

$$
K_{822}=-\varepsilon\left\langle\partial_{x}^{\alpha} \Delta \phi_{R}^{\varepsilon}, \frac{\varepsilon}{n} \partial_{t} \Delta \partial_{x}^{\alpha} \phi_{R}^{\varepsilon}\right\rangle_{0}
$$

By integration by parts in time, we obtain

$$
K_{822}=-\frac{1}{2} \frac{d}{d t}\left\langle\partial_{x}^{\alpha} \Delta \phi_{R}^{\varepsilon}, \frac{\varepsilon^{2}}{n} \partial_{x}^{\alpha} \Delta \phi_{R}^{\varepsilon}\right\rangle_{0}+\frac{1}{2}\left\langle\partial_{x}^{\alpha} \Delta \phi_{R}^{\varepsilon}, \partial_{t}\left(\frac{\varepsilon^{2}}{n}\right) \partial_{x}^{\alpha} \Delta \phi_{R}^{\varepsilon}\right\rangle_{0} .
$$

Similar to (4.36), it is estimated that

$$
K_{822} \leq-\frac{1}{2} \frac{d}{d t}\left\langle\partial_{x}^{\alpha} \Delta \phi_{R}^{\varepsilon}, \frac{\varepsilon^{2}}{n} \partial_{x}^{\alpha} \Delta \phi_{R}^{\varepsilon}\right\rangle_{0}+C\left(1+\varepsilon\left\|\left(\mathbf{u}_{R}^{\varepsilon}, \phi_{R}^{\varepsilon}\right)\right\| \|_{3}\right)\left\|\phi_{R}^{\varepsilon}\right\|_{s^{\prime}}^{2} .
$$

Estimate of $K_{823}$. Recall that in 4.33

$$
K_{823}=\left\langle\partial_{x}^{\alpha} \Delta \phi_{R}^{\varepsilon}, \frac{\varepsilon^{2}}{n} \partial_{t} \partial_{x}^{\alpha}\left(\phi^{(1)} \phi_{R}^{\varepsilon}\right)\right\rangle_{0} .
$$

By integrating by parts and using the commutator, we can rewrite

$$
\begin{aligned}
K_{823}= & -\left\langle\partial_{x}^{\alpha} \nabla \phi_{R}^{\varepsilon}, \nabla\left(\frac{\varepsilon^{2}}{n}\right) \partial_{x}^{\alpha}\left(\phi^{(1)} \partial_{t} \phi_{R}^{\varepsilon}\right)\right\rangle_{0}-\left\langle\partial_{x}^{\alpha} \nabla \phi_{R}^{\varepsilon}, \nabla\left(\frac{\varepsilon^{2}}{n}\right) \partial_{x}^{\alpha}\left(\partial_{t} \phi^{(1)} \phi_{R}^{\varepsilon}\right)\right\rangle_{0} \\
& -\left\langle\partial_{x}^{\alpha} \nabla \phi_{R}^{\varepsilon},\left(\frac{\varepsilon^{2} \phi^{(1)}}{n}\right) \partial_{t} \partial_{x}^{\alpha} \nabla \phi_{R}^{\varepsilon}\right\rangle_{0}-\left\langle\partial_{x}^{\alpha} \nabla \phi_{R}^{\varepsilon},\left(\frac{\varepsilon^{2}}{n}\right)\left[\partial_{x}^{\alpha} \nabla, \phi^{(1)}\right] \partial_{t} \phi_{R}^{\varepsilon}\right\rangle_{0} \\
& -\left\langle\partial_{x}^{\alpha} \nabla \phi_{R}^{\varepsilon},\left(\frac{\varepsilon^{2}}{n}\right) \partial_{x}^{\alpha} \nabla\left(\partial_{t} \phi^{(1)} \phi_{R}^{\varepsilon}\right)\right\rangle_{0} \\
= & : K_{8231}+K_{8232}+K_{8233}+K_{8234}+K_{8235} .
\end{aligned}
$$


We note that from 4.37, we have

$$
\left\|\nabla\left(\frac{\varepsilon^{2}}{n}\right)\right\|_{L^{\infty}} \leq C \varepsilon^{3}\left(1+\varepsilon\left\|\nabla n_{R}^{\varepsilon}\right\|_{L^{\infty}}\right) .
$$

Using the multiplicative estimates $[$ C.1 in Lemma C.1] we obtain

$$
K_{8231} \leq C \varepsilon^{3}\left(1+\varepsilon\left\|\nabla n_{R}^{\varepsilon}\right\|_{L^{\infty}}\right)\left\|\partial_{x}^{\alpha} \nabla \phi_{R}^{\varepsilon}\right\|_{L^{2}}\left\{\left\|\partial_{t} \phi_{R}^{\varepsilon}\right\|_{H^{k}}\left\|\phi^{(1)}\right\|_{L^{\infty}}+\left\|\partial_{t} \phi_{R}^{\varepsilon}\right\|_{L^{\infty}}\left\|\phi^{(1)}\right\|_{H^{k}}\right\}
$$

Since

$$
\left\|\partial_{t} \phi_{R}^{\varepsilon}\right\|_{H^{k}} \leq\left\|\partial_{t} \phi_{R}^{\varepsilon}\right\|_{H^{k-1}}+\left\|\partial_{t} \nabla \phi_{R}^{\varepsilon}\right\|_{H^{k-1}},
$$

we obtain

$$
\begin{aligned}
K_{8231} \leq & C\left(1+\varepsilon\left\|\nabla n_{R}^{\varepsilon}\right\|_{L^{\infty}}\right) \\
& \times\left\{\varepsilon\left\|\nabla \phi_{R}^{\varepsilon}\right\|_{H^{k}}^{2}+\left\|\varepsilon \partial_{t} \phi_{R}^{\varepsilon}\right\|_{H^{k-1}}^{2}+\varepsilon\left\|\varepsilon \partial_{t} \nabla \phi_{R}^{\varepsilon}\right\|_{H^{k-1}}^{2}+\left\|\varepsilon \partial_{t} \phi_{R}^{\varepsilon}\right\|_{H^{2}}^{2}\right\} \\
\leq & C\left(1+\varepsilon\left\|\phi_{R}^{\varepsilon}\right\|_{3}\right)\left\{1+\left\|\left(\mathbf{u}_{R}^{\varepsilon}, \phi_{R}^{\varepsilon}\right)\right\|_{s^{\prime}}^{2}\right\} .
\end{aligned}
$$

where we have used Lemma 3.1 and Corollary 3.1

Similarly, we have

$$
K_{8232} \leq C\left(1+\varepsilon\left\|\phi_{R}^{\varepsilon}\right\|_{3}\right)\left(1+\left\|\phi_{R}^{\varepsilon}\right\|_{s^{\prime}}^{2}\right) .
$$

Now we estimate $K_{8233}$. By integrating by parts in time, we obtain

$$
\begin{aligned}
K_{8233} & =-\frac{1}{2} \frac{d}{d t}\left\langle\partial_{x}^{\alpha} \nabla \phi_{R}^{\varepsilon},\left(\frac{\varepsilon^{2} \phi^{(1)}}{n}\right) \partial_{x}^{\alpha} \nabla \phi_{R}^{\varepsilon}\right\rangle_{0}+\frac{1}{2}\left\langle\partial_{x}^{\alpha} \nabla \phi_{R}^{\varepsilon}, \partial_{t}\left(\frac{\varepsilon^{2} \phi^{(1)}}{n}\right) \partial_{x}^{\alpha} \nabla \phi_{R}^{\varepsilon}\right\rangle_{0} \\
& \leq-\frac{1}{2} \frac{d}{d t}\left\langle\partial_{x}^{\alpha} \nabla \phi_{R}^{\varepsilon},\left(\frac{\varepsilon^{2} \phi^{(1)}}{n}\right) \partial_{x}^{\alpha} \nabla \phi_{R}^{\varepsilon}\right\rangle_{0}+C\left(1+\varepsilon\left\|\left(\mathbf{u}_{R}^{\varepsilon}, \phi_{R}^{\varepsilon}\right)\right\| \|_{3}\right)\left\|\phi_{R}^{\varepsilon}\right\|_{s^{\prime}}^{2} .
\end{aligned}
$$

For the term $K_{8234}$ in 4.33), we have

$$
\begin{aligned}
K_{8234} & =-\left\langle\partial_{x}^{\alpha} \nabla \phi_{R}^{\varepsilon},\left(\frac{\varepsilon^{2}}{n}\right)\left[\partial_{x}^{\alpha} \nabla, \phi^{(1)}\right] \partial_{t} \phi_{R}^{\varepsilon}\right\rangle_{0} \\
& \leq C \varepsilon^{2}\left\|\partial_{x}^{\alpha} \nabla \phi_{R}^{\varepsilon}\right\|_{L^{2}}\left\{\left\|\partial_{t} \phi_{R}^{\varepsilon}\right\|_{H^{k}}\left\|\phi^{(1)}\right\|_{L^{\infty}}+\left\|\partial_{t} \phi_{R}^{\varepsilon}\right\|_{L^{\infty}}\left\|\phi^{(1)}\right\|_{H^{k+1}}\right\} \\
& \leq C \varepsilon\left\|\partial_{x}^{\alpha} \nabla \phi_{R}^{\varepsilon}\right\|_{L^{2}}^{2}+C\left\{\varepsilon\left\|\varepsilon \partial_{t} \phi_{R}^{\varepsilon}\right\|_{H^{k}}^{2}+\varepsilon\left\|\varepsilon \partial_{t} \phi_{R}^{\varepsilon}\right\|_{H^{2}}^{2}\right\} .
\end{aligned}
$$

Similarly to the estimate of $K_{8231}$ in 4.47), we have

$$
K_{8234} \leq C\left(1+\varepsilon\left\|\phi_{R}^{\varepsilon}\right\|_{3}\right)\left\{1+\left\|\left(\mathbf{u}_{R}^{\varepsilon}, \phi_{R}^{\varepsilon}\right)\right\|_{s^{\prime}}^{2}\right\} .
$$

For $K_{8235}$, by using multiplicative estimates in $[$ C.1 , we have

$$
\begin{aligned}
K_{8235}= & -\left\langle\partial_{x}^{\alpha} \nabla \phi_{R}^{\varepsilon},\left(\frac{\varepsilon^{2}}{n}\right) \partial_{x}^{\alpha}\left(\partial_{t} \phi^{(1)} \nabla \phi_{R}^{\varepsilon}\right)\right\rangle_{0}-\left\langle\partial_{x}^{\alpha} \nabla \phi_{R}^{\varepsilon},\left(\frac{\varepsilon^{2}}{n}\right) \partial_{x}^{\alpha}\left(\nabla \partial_{t} \phi^{(1)} \phi_{R}^{\varepsilon}\right)\right\rangle_{0} \\
\leq & C \varepsilon^{2}\left\|\partial_{x}^{\alpha} \nabla \phi_{R}^{\varepsilon}\right\|_{L^{2}}\left\{\left\|\nabla \phi_{R}^{\varepsilon}\right\|_{H^{k}}\left\|\partial_{t} \phi^{(1)}\right\|_{L^{\infty}}+\left\|\phi_{R}^{\varepsilon}\right\|_{L^{\infty}}\left\|\partial_{t} \phi^{(1)}\right\|_{H^{k+1}}\right. \\
& \left.+\left\|\phi_{R}^{\varepsilon}\right\|_{H^{k}}\left\|\partial_{t} \nabla \phi^{(1)}\right\|_{L^{\infty}}+\left\|\phi_{R}^{\varepsilon}\right\|_{L^{\infty}}\left\|\partial_{t} \nabla \phi^{(1)}\right\|_{H^{k+1}}\right\} \\
\leq & C \varepsilon\left\|\partial_{x}^{\alpha} \nabla \phi_{R}^{\varepsilon}\right\|_{L^{2}}^{2}+C\left\{\varepsilon\left\|\nabla \phi_{R}^{\varepsilon}\right\|_{H^{k}}^{2}+\left\|\phi_{R}^{\varepsilon}\right\|_{H^{k}}^{2}+\left\|\phi_{R}^{\varepsilon}\right\|_{H^{3}}^{2}\right\} \\
\leq & C\left\|\phi_{R}^{\varepsilon}\right\|_{s^{\prime}}^{2},
\end{aligned}
$$

where $k \leq s^{\prime}$ and $s^{\prime} \geq 3$. 
From 4.44, by adding 4.47 to 4.51) together, we obtain

$$
\begin{aligned}
K_{823} \leq & -\frac{1}{2} \frac{d}{d t}\left\langle\partial_{x}^{\alpha} \nabla \phi_{R}^{\varepsilon},\left(\frac{\varepsilon^{2} \phi^{(1)}}{n}\right) \partial_{x}^{\alpha} \nabla \phi_{R}^{\varepsilon}\right\rangle_{0} \\
& +C\left(1+\varepsilon\left\|\left(\mathbf{u}_{R}^{\varepsilon}, \phi_{R}^{\varepsilon}\right)\right\| \|_{3}\right)\left\{1+\left\|\left(\mathbf{u}_{R}^{\varepsilon}, \phi_{R}^{\varepsilon}\right)\right\|_{s^{\prime}}^{2}\right\} .
\end{aligned}
$$

Estimate of $K_{824}$. Recall that $K_{824}$ is defined in 4.33

$$
\begin{aligned}
K_{824}= & \left\langle\partial_{x}^{\alpha} \Delta \phi_{R}^{\varepsilon}, \frac{\varepsilon^{3}}{n} \partial_{x}^{\alpha}\left(\phi_{R}^{\varepsilon} \partial_{t} \phi_{R}^{\varepsilon}\right)\right\rangle_{0} \\
= & -\left\langle\partial_{x}^{\alpha} \nabla \phi_{R}^{\varepsilon}, \frac{\varepsilon^{3} \phi_{R}^{\varepsilon}}{n} \partial_{t} \partial_{x}^{\alpha} \nabla \phi_{R}^{\varepsilon}\right\rangle_{0}-\left\langle\partial_{x}^{\alpha} \nabla \phi_{R}^{\varepsilon}, \frac{\varepsilon^{3}}{n}\left[\partial_{x}^{\alpha} \nabla, \phi_{R}^{\varepsilon}\right] \partial_{t} \phi_{R}^{\varepsilon}\right\rangle_{0} \\
& -\left\langle\partial_{x}^{\alpha} \nabla \phi_{R}^{\varepsilon}, \frac{\varepsilon^{3}}{n} \partial_{x}^{\alpha}\left(\nabla \phi_{R}^{\varepsilon} \partial_{t} \phi_{R}^{\varepsilon}\right)\right\rangle_{0} \\
= & : K_{8241}+K_{8242}+K_{8243} .
\end{aligned}
$$

For the term $K_{8241}$, by integrating by parts in time, we obtain

$$
K_{8241}=-\frac{1}{2} \frac{d}{d t}\left\langle\partial_{x}^{\alpha} \nabla \phi_{R}^{\varepsilon}, \frac{\varepsilon^{3} \phi_{R}^{\varepsilon}}{n} \partial_{x}^{\alpha} \nabla \phi_{R}^{\varepsilon}\right\rangle_{0}+\frac{1}{2}\left\langle\partial_{x}^{\alpha} \nabla \phi_{R}^{\varepsilon}, \partial_{t}\left(\frac{\varepsilon^{3} \phi_{R}^{\varepsilon}}{n}\right) \partial_{x}^{\alpha} \nabla \phi_{R}^{\varepsilon}\right\rangle_{0}
$$

By using Hölder inequality, Lemma 3.1, 3.2 and Corollary 3.1, we obtain

$$
\begin{aligned}
\left\|\partial_{t}\left(\frac{\varepsilon^{2} \phi_{R}^{\varepsilon}}{n}\right)\right\|_{L^{\infty}} & \leq C \varepsilon^{2}\left\|\partial_{t} \phi_{R}^{\varepsilon}\right\|_{L^{\infty}}+C \varepsilon^{3}\left\|\phi_{R}^{\varepsilon}\right\|_{L^{\infty}}\left\|\partial_{t} \widetilde{n}_{R}^{\varepsilon}\right\|_{L^{\infty}}+C \varepsilon^{4}\left\|\phi_{R}^{\varepsilon}\right\|_{L^{\infty}}\left\|\partial_{t} n_{R}^{\varepsilon}\right\|_{L^{\infty}} \\
& \leq C+C \varepsilon^{2}\left\|\varepsilon \partial_{t} \phi_{R}^{\varepsilon}\right\|_{H^{2}}^{2}+C \varepsilon^{2}\left\|\phi_{R}^{\varepsilon}\right\|_{H^{2}}^{2}+C \varepsilon^{2}\left\|\varepsilon \partial_{t} n_{R}^{\varepsilon}\right\|_{H^{2}}^{2} \\
& \leq C+C \varepsilon^{2}\left\|\left(\mathbf{u}_{R}^{\varepsilon}, \phi_{R}^{\varepsilon}\right)\right\|_{3}^{2} .
\end{aligned}
$$

Therefore, $K_{8241}$ in 4.54 is estimated as

$$
K_{8241}=-\frac{1}{2} \frac{d}{d t}\left\langle\partial_{x}^{\alpha} \nabla \phi_{R}^{\varepsilon}, \frac{\varepsilon^{3} \phi_{R}^{\varepsilon}}{n} \partial_{x}^{\alpha} \nabla \phi_{R}^{\varepsilon}\right\rangle_{0}+C\left(1+\varepsilon^{2}\left\|\left(\mathbf{u}_{R}^{\varepsilon}, \phi_{R}^{\varepsilon}\right)\right\| \|_{3}^{2}\right)\left\{\varepsilon\left\|\partial_{x}^{\alpha} \nabla \phi_{R}^{\varepsilon}\right\|_{L^{2}}^{2}\right\} .
$$

For the term $K_{8242}$ in 4.53, by commutator estimates in (C.1), we have

$$
\begin{aligned}
K_{8242} & =-\left\langle\partial_{x}^{\alpha} \nabla \phi_{R}^{\varepsilon}, \frac{\varepsilon^{3}}{n}\left[\partial_{x}^{\alpha} \nabla, \phi_{R}^{\varepsilon}\right] \partial_{t} \phi_{R}^{\varepsilon}\right\rangle_{0} \\
& \leq C \varepsilon^{3}\left\|\partial_{x}^{\alpha} \nabla \phi_{R}^{\varepsilon}\right\|_{L^{2}}\left\{\left\|\partial_{t} \phi_{R}^{\varepsilon}\right\|_{H^{k}}\left\|\phi_{R}^{\varepsilon}\right\|_{L^{\infty}}+\left\|\partial_{t} \phi_{R}^{\varepsilon}\right\|_{L^{\infty}}\left\|\phi_{R}^{\varepsilon}\right\|_{H^{k+1}}\right\} \\
& \leq C \varepsilon\left\|\nabla \phi_{R}^{\varepsilon}\right\|_{H^{k}}^{2}+C\left(\varepsilon^{2}\left\|\phi_{R}^{\varepsilon}\right\|_{H^{3}}^{2}+\varepsilon^{2}\left\|\varepsilon \partial_{t} \phi_{R}^{\varepsilon}\right\|_{H^{2}}^{2}\right)\left(\varepsilon\left\|\varepsilon \partial_{t} \phi_{R}^{\varepsilon}\right\|_{H^{k}}^{2}+\varepsilon\left\|\phi_{R}^{\varepsilon}\right\|_{H^{k+1}}^{2}\right) .
\end{aligned}
$$

Using 4.46, Lemma 3.1 and Corollary 3.1, we then have

$$
K_{8242} \leq C\left(1+\varepsilon^{2}\left\|\left(\mathbf{u}_{R}^{\varepsilon}, \phi_{R}^{\varepsilon}\right)\right\| \|_{3}^{2}\right)\left\|\left(\mathbf{u}_{R}^{\varepsilon}, \phi_{R}^{\varepsilon}\right)\right\|_{s^{\prime}}^{2} .
$$

For the term $K_{8243}$ in 4.53, by multiplicative estimates in (C.1), we have

$$
\begin{aligned}
K_{8243} & =-\left\langle\partial_{x}^{\alpha} \nabla \phi_{R}^{\varepsilon}, \frac{\varepsilon^{3}}{n} \partial_{x}^{\alpha}\left(\nabla \phi_{R}^{\varepsilon} \partial_{t} \phi_{R}^{\varepsilon}\right)\right\rangle_{0} \\
& \leq C \varepsilon^{3}\left\|\partial_{x}^{\alpha} \nabla \phi_{R}^{\varepsilon}\right\|_{L^{2}}\left\{\left\|\partial_{t} \phi_{R}^{\varepsilon}\right\|_{H^{k}}\left\|\nabla \phi_{R}^{\varepsilon}\right\|_{L^{\infty}}+\left\|\partial_{t} \phi_{R}^{\varepsilon}\right\|_{L^{\infty}}\left\|\nabla \phi_{R}^{\varepsilon}\right\|_{H^{k}}\right\} \\
& \leq C\left(1+\varepsilon^{2}\left\|\left(\mathbf{u}_{R}^{\varepsilon}, \phi_{R}^{\varepsilon}\right)\right\|_{3}^{2}\right)\left\|\left(\mathbf{u}_{R}^{\varepsilon}, \phi_{R}^{\varepsilon}\right)\right\|_{s^{\prime}}^{2},
\end{aligned}
$$

where in the last inequality, we have used the same estimates as in (4.58). 
From (4.53), adding (4.54, (4.56) and 4.58), we obtain

$$
K_{824} \leq-\frac{1}{2} \frac{d}{d t}\left\langle\partial_{x}^{\alpha} \nabla \phi_{R}^{\varepsilon}, \frac{\varepsilon^{3} \phi_{R}^{\varepsilon}}{n} \partial_{x}^{\alpha} \nabla \phi_{R}^{\varepsilon}\right\rangle_{0}+C \varepsilon\left(1+\varepsilon^{2}\left\|\left(\mathbf{u}_{R}^{\varepsilon}, \phi_{R}^{\varepsilon}\right)\right\| \|_{3}^{2}\right)\left\|\left(\mathbf{u}_{R}^{\varepsilon}, \phi_{R}^{\varepsilon}\right)\right\|_{s^{\prime}}^{2}
$$

Estimate of $K_{825}$. Recall that $K_{825}$ is defined in 4.33). By 2.23) in Lemma2.1 we have

$$
\begin{aligned}
K_{825} & \leq C \varepsilon^{2}\left\|\partial_{x}^{\alpha} \Delta \phi_{R}^{\varepsilon}\right\|^{2}+\varepsilon^{4}\left\|\partial_{t} \partial_{x}^{\alpha} R_{\phi}\right\|^{2} \\
& \leq C \varepsilon^{2}\left\|\partial_{x}^{\alpha} \Delta \phi_{R}^{\varepsilon}\right\|^{2}+C_{1}\left(\sqrt{\varepsilon}\left\|\phi_{R}^{\varepsilon}\right\|_{H^{\delta}}\right)\left(1+\varepsilon^{2}\left\|\varepsilon \partial_{t} \phi_{R}^{\varepsilon}\right\|_{H^{k}}^{2}\right),
\end{aligned}
$$

where $\delta=\max \{2, k-1\}$ in Lemma2.1. Furthermore, when $0<\varepsilon<\varepsilon_{1}$,

$$
\begin{aligned}
\varepsilon^{2}\left\|\varepsilon \partial_{t} \phi_{R}^{\varepsilon}\right\|_{H^{k}}^{2} & \leq \varepsilon^{2}\left\|\varepsilon \partial_{t} \nabla \phi_{R}^{\varepsilon}\right\|_{H^{k-1}}^{2}+\varepsilon^{2}\left\|\varepsilon \partial_{t} \phi_{R}^{\varepsilon}\right\|_{H^{k-1}}^{2} \\
& \leq C\left(1+\left\|\left(\mathbf{u}_{R}^{\varepsilon}, \phi_{R}^{\varepsilon}\right)\right\|_{S^{\prime}}^{2}\right)
\end{aligned}
$$

where we have used 3.25) in Corollary 3.1 in the last inequality. It then follows from 4.61) that

$$
K_{825} \leq C_{1}\left(\sqrt{\varepsilon}\left\|\phi_{R}^{\varepsilon}\right\|_{H^{\delta}}\right)\left\{1+\left\|\left(\mathbf{u}_{R}^{\varepsilon}, \phi_{R}^{\varepsilon}\right)\right\|_{s^{\prime}}^{2}\right\}
$$

where $\delta=\max \{2, k-1\} \leq s^{\prime}-1$.

The proof of Lemma 4.2 is complete by adding (4.39), 4.42), 4.52, 4.60) and 4.63) together.

Proof of Theorem 2.1 for $T_{i}=0$. From (4.1), there exists some $\varepsilon_{1}>0$ such that $1 / 2 \leq 1+\varepsilon \phi^{(1)}+\varepsilon^{2} \phi_{R}^{\varepsilon} \leq$ $3 / 2$. By adding inequalities (4.3) and (4.5), then integrating over $[0, t]$ and taking summation over $|\alpha|=k$ for $0 \leq k \leq s^{\prime}$, we obtain

$$
\left\|\left(\mathbf{u}_{R}^{\varepsilon}, \phi_{R}^{\varepsilon}\right)\right\|_{s^{\prime}}^{2} \leq C C_{\varepsilon}(0)+C C_{1} \int_{0}^{t}\left(C_{1}+\varepsilon\left\|\left(\mathbf{u}_{R}^{\varepsilon}, n_{R}^{\varepsilon}, \phi_{R}^{\varepsilon}\right)\right\| \|_{s^{\prime}}^{2}\right)\left\{1+\left\|\left(\mathbf{u}_{R}^{\varepsilon}, n_{R}^{\varepsilon}, \phi_{R}^{\varepsilon}\right)\right\|_{s^{\prime}}^{2}\right\} d r
$$

where $C_{\varepsilon}(0)=\left\|\left(\mathbf{u}_{R}^{\varepsilon}, \phi_{R}^{\varepsilon}\right)(0)\right\|_{s^{\prime}}^{2}$. Recalling 3.1), we know that there exists some constant $0<\varepsilon_{0}<\varepsilon_{1}$ such that $\varepsilon\left\|\left(\mathbf{u}_{R}^{\varepsilon}, n_{R}^{\varepsilon}, \phi_{R}^{\varepsilon}\right)\right\|_{H^{s^{\prime}}}^{2} \leq 1$. Since $C_{1}=C_{1}\left(\sqrt{\varepsilon}\left\|n_{R}^{\varepsilon}\right\|_{H^{s^{\prime}}}\right)$ and is nondecreasing, we know that $C_{1} \leq C_{1}(1)$ when $0<\varepsilon<\varepsilon_{0}$. Therefore, there exists some constant $C_{3}>1$ such that

$$
\left\|\left(\mathbf{u}_{R}^{\varepsilon}, \phi_{R}^{\varepsilon}\right)\right\|_{s^{\prime}}^{2} \leq C_{3} C_{\varepsilon}(0)+C_{3} \int_{0}^{t}\left\{1+\left\|\left(\mathbf{u}_{R}^{\varepsilon}, \phi_{R}^{\varepsilon}\right)\right\|_{s^{\prime}}^{2}\right\} d r
$$

On the other hand, from Lemma 3.1, there exists some constant $C_{4} \geq 1$ such that for any $0<\varepsilon<\varepsilon_{0}$,

$$
\left\|n_{R}^{\varepsilon}\right\|_{H^{s^{\prime}}}^{2} \leq C_{4}\left(1+\left\|\phi_{R}^{\varepsilon}\right\|_{s^{\prime}}^{2}\right)
$$

Let $C_{0}^{\prime}=\sup _{0<\varepsilon<1} C_{\varepsilon}(0)$. Given given $0<\tau_{0}<\tau_{*}$, we let $\tilde{C}$ in 3.1 satisfy $\tilde{C} \geq 2 C_{4}\left(1+C_{3} C_{0}^{\prime}\right) e^{C_{3} \tau_{0}}$, then by Gronwall inequality,

$$
\sup _{0 \leq t \leq \tau_{0}}\left\|\left(\mathbf{u}_{R}^{\varepsilon}, \phi_{R}^{\varepsilon}\right)\right\|_{s^{\prime}}^{2} \leq\left(1+C_{3} C_{0}^{\prime}\right) e^{C_{3} \tau_{0}} \leq \tilde{C}
$$

and from 4.66

$$
\sup _{0 \leq t \leq \tau_{0}}\left\|n_{R}^{\varepsilon}\right\|_{H^{s^{\prime}}}^{2} \leq C_{4}\left\{1+\left(1+C_{3} C_{0}^{\prime}\right) e^{C_{3} \tau_{0}}\right\} \leq \tilde{C}
$$

It is then standard to obtain uniform estimates for $\left\|\left(n_{R}^{\varepsilon}, \mathbf{u}_{R}^{\varepsilon}, \phi_{R}^{\varepsilon}\right)\right\| \|_{s^{\prime}}$ independent of $\varepsilon$ by the continuity method. The proof is complete for the case $T_{i}=0$. 


\section{A Proof of Proposition 2.3 and Lemma 2.1}

Proof of Proposition 2.3 We need to derive the remainder system 2.21). We first consider the remainder equation 2.21a). Plugging the expansion of $n$ and $\mathbf{u}=\left(u_{1}, u_{2}\right)$ in 2.19 ) into the 2.2a), we obtain a polynomial equation of $\varepsilon$, whose coefficients depend on $n^{(i)}, \mathbf{u}^{(i)}, n_{R}^{\varepsilon}$ and $\mathbf{u}_{R}^{\varepsilon}$. Subtracting $\{\varepsilon \times \sqrt{2.4 a}\}+$ $\varepsilon^{2} \times\left[2.7 \mathrm{a}, \varepsilon^{3} \times 2.15 \mathrm{a}\right\}$ from this polynomial, we obtain the following equation

$$
\varepsilon^{4} \partial_{t} n^{(3)}+\varepsilon^{3} \partial_{t} n_{R}^{\varepsilon}-\varepsilon^{2} \partial_{x} n_{R}^{\varepsilon}+A+B=0,
$$

where

$$
\begin{aligned}
& A=\varepsilon^{4} \sum_{\substack{i, j \geq 1 \\
i+j \geq 4}} \varepsilon^{i+j-4} \partial_{x}\left(n^{(i)} u_{1}^{(j)}\right)+\varepsilon^{3}\left\{u_{1}^{\varepsilon} \partial_{x} \widetilde{n}+\partial_{x} \widetilde{u}_{1} n_{R}^{\varepsilon}\right\}+\varepsilon^{2}\left\{n \partial_{x} u_{1}^{\varepsilon}+u_{1} \partial_{x} n_{R}^{\varepsilon}\right\}, \\
& B=\varepsilon^{4} \sum_{\substack{i, l \geq 1 \\
i+l \geq 4}} \varepsilon^{i+l+\frac{1}{2}-4} \partial_{x}\left(n^{(i)} u_{2}^{(l)}\right)+\varepsilon^{3}\left\{u_{2}^{\varepsilon} \partial_{x} \widetilde{n}+\partial_{x} \widetilde{u}_{2} n_{R}^{\varepsilon}\right\}+\varepsilon^{2}\left\{n \partial_{x} u_{2}^{\varepsilon}+u_{2} \partial_{x} n_{R}^{\varepsilon}\right\} .
\end{aligned}
$$

Rearranging and dividing (A.1) by $\varepsilon^{3}$, we obtain $2.21 \mathrm{a}$, where

$$
R_{n}=\partial_{t} n^{(3)}+\sum_{\substack{i, j \geq 1 \\ i+j \geq 4}} \varepsilon^{i+j-4} \partial_{x}\left(n^{(i)} u_{1}^{(j)}\right)+\sum_{\substack{i, l \geq 1 \\ i+l \geq 4}} \varepsilon^{i+l+\frac{1}{2}-4} \partial_{x}\left(n^{(i)} u_{2}^{(l)}\right) .
$$

The derivation of (2.21b) and $(2.21 c)$ is similar. Subtracting $\left\{\varepsilon \times(2.4 b)+\varepsilon^{2} \times(2.7 b)+\varepsilon^{3} \times(2.15 b)\right\}$ from the equation of $2.2 \mathrm{~b}$, , we obtain the remainder equation $2.21 \mathrm{~b})$. We only derive the remainder terms of the pressure term $T_{i} \partial_{x_{1}} n / n$. After subtracting, we obtain

$$
\begin{aligned}
T_{i} \frac{\partial_{x_{1}} n}{n}- & T_{i}\left\{\varepsilon \partial_{x_{1}} n^{(1)}+\varepsilon^{2}\left(\partial_{x_{1}} n^{(2)}-n^{(1)} \partial_{x_{1}} n^{(1)}\right)\right. \\
& \left.+\varepsilon^{3}\left(\partial_{x_{1}} n^{(3)}+\partial_{x_{1}} n^{(1)}\left(\frac{1}{2}\left(n^{(1)}\right)^{2}-n^{(2)}\right)-\partial_{x_{1}} n^{(2)} n^{(1)}\right)\right\}
\end{aligned}
$$

After divided by $\varepsilon^{3}$, A.3) can be rearranged into

$$
T_{i} \frac{\partial_{x_{1}} n_{R}^{\varepsilon}}{\varepsilon n}-T_{i} \frac{p_{1}}{n} n_{R}^{\varepsilon}-T_{i} \frac{\varepsilon R_{T 1}}{n},
$$

where $p_{1}$ and $R_{T 1}$ are finite combinations of $n^{(1)}, n^{(2)}$ and $n^{(3)}$ only. The expression of $\mathbf{R}_{\mathbf{u}}$ depends only on $\mathbf{u}^{(i)}$ and $\phi^{i}$ and can be derived similarly to the derivation of $R_{n}$ in A.2.

The derivation of (2.21d is slightly different, where the remainder $R_{\phi}$ depends on $\phi_{R}^{\varepsilon}$. Recall $\phi=$ $\varepsilon \phi^{(1)}+\varepsilon^{2} \phi^{(2)}+\varepsilon^{3} \phi^{(3)}+\varepsilon^{2} \phi_{R}^{\varepsilon}$ in 2.19d). Consider the Taylor expansion in the integral form

$$
\begin{aligned}
e^{\phi}=1 & +\left(\varepsilon \widetilde{\phi}+\varepsilon^{2} \phi_{R}^{\varepsilon}\right)+\frac{1}{2 !}\left(\varepsilon \widetilde{\phi}+\varepsilon^{2} \phi_{R}^{\varepsilon}\right)^{2}+\frac{1}{3 !}\left(\varepsilon \widetilde{\phi}+\varepsilon^{2} \phi_{R}^{\varepsilon}\right)^{3} \\
& +\frac{1}{3 !} \int_{0}^{1} e^{\theta \phi}(1-\theta)^{3} d \theta\left(\varepsilon \widetilde{\phi}+\varepsilon^{2} \phi_{R}^{\varepsilon}\right)^{4} .
\end{aligned}
$$

Subtracting $\left\{\varepsilon \times(\underline{2.4 \mathrm{c}})+\varepsilon^{2} \times(\underline{2.7 \mathrm{c}})+\varepsilon^{3} \times \underline{2.15 \mathrm{c}}\right\}$ from $\underline{2.2 \mathrm{~d}}$, we have

$$
\begin{aligned}
& \varepsilon^{3}\left(\partial_{x_{1}}^{2}+\varepsilon \partial_{x_{2}}^{2}\right) \phi_{R}^{\varepsilon}+\varepsilon^{4}\left\{\left(\partial_{x_{1}}^{2}+\varepsilon \partial_{x_{2}}^{2}\right) \phi^{(3)}+\partial_{x_{2}}^{2} \phi^{(2)}\right\} \\
= & \varepsilon^{4} \hat{R}_{\phi}+\varepsilon^{2} \phi_{R}^{\varepsilon}+\varepsilon^{3} \widetilde{\phi} \phi_{R}^{\varepsilon}+\frac{1}{2} \varepsilon^{4}\left(\phi_{R}^{\varepsilon}\right)^{2}+\frac{1}{2} \varepsilon^{4}(\widetilde{\phi})^{2} \phi_{R}^{\varepsilon}+\frac{1}{2} \varepsilon^{5} \widetilde{\phi}\left(\phi_{R}^{\varepsilon}\right)^{2} \\
& +\frac{1}{3 !} \varepsilon^{6}\left(\phi_{R}^{\varepsilon}\right)^{3}+\varepsilon^{4} \frac{1}{3 !} \int_{0}^{1} e^{\theta \phi}(1-\theta)^{3} d \theta\left(\widetilde{\phi}+\varepsilon \phi_{R}^{\varepsilon}\right)^{4}-\varepsilon^{2} n_{R}^{\varepsilon},
\end{aligned}
$$


where $\hat{R}_{\phi}$ depends only $\phi^{(1)}, \phi^{(2)}$ and $\phi^{(3)}$. After divided by $\varepsilon^{2}$, A.5 can be rewritten in the form

$$
\varepsilon\left(\partial_{x_{1}}^{2}+\varepsilon \partial_{x_{2}}^{2}\right) \phi_{R}^{\varepsilon}=\phi_{R}^{\varepsilon}-n_{R}^{\varepsilon}+\varepsilon \phi^{(1)} \phi_{R}^{\varepsilon}+\varepsilon^{3 / 2} R_{\phi}^{\prime}+\varepsilon^{2} R_{\phi}^{\prime \prime},
$$

where $R_{\phi}^{\prime \prime}=\hat{R}_{\phi}-\left\{\left(\partial_{x_{1}}^{2}+\varepsilon \partial_{x_{2}}^{2}\right) \phi^{(3)}+\partial_{x_{2}}^{2} \phi^{(2)}\right\}$ and $R_{\phi}^{\prime}=F\left(\sqrt{\varepsilon} \phi_{R}^{\varepsilon}\right) \phi_{R}^{\varepsilon}$ for some function of $F$ depending on $\sqrt{\varepsilon} \phi_{R}^{\varepsilon}$. Letting $R_{\phi}=R_{\phi}^{\prime}+\sqrt{\varepsilon} R_{\phi}^{\prime \prime}$, we obtain (2.21d).

From (A.5), it is obvious that 2.21d can be written in an equivalent form

$$
\varepsilon\left(\partial_{x_{1}}^{2}+\varepsilon \partial_{x_{2}}^{2}\right) \phi_{R}^{\varepsilon}=\phi_{R}^{\varepsilon}-n_{R}^{\varepsilon}+\varepsilon \phi^{(1)} \phi_{R}^{\varepsilon}+\frac{\varepsilon^{2}}{2}\left(\phi_{R}^{\varepsilon}\right)^{2}+\varepsilon^{2} \bar{R}_{\phi},
$$

where $\bar{R}_{\phi}$ is also of the form of $R_{\phi}$ and satisfies the same estimates of Lemma 2.1

The proof of Proposition 2.3 is complete.

Proof of Lemma 2.1 We mainly consider the estimate for the integral term in A.5, which has an important contribution to the remainder term $R_{\phi}^{\prime}$, while the other contributions from (A.5) can be estimated similarly. Let $\alpha=0$. By Hölder inequality and Sobolev embedding, we have

$$
\begin{aligned}
\|I\|_{L^{2}} & \leq C e^{\|\phi\|_{L^{\infty}}}\left\|\widetilde{\phi}+\varepsilon \phi_{R}^{\varepsilon}\right\|_{L^{\infty}}^{3}\left\|\widetilde{\phi}+\varepsilon \phi_{R}^{\varepsilon}\right\|_{L^{2}} \\
& \leq C\left(\varepsilon\left\|\phi_{R}^{\varepsilon}\right\|_{L^{\infty}}\right)\left(\|\widetilde{\phi}\|_{L^{2}}+\left\|\phi_{R}^{\varepsilon}\right\|_{L^{2}}\right) \\
& \leq C\left(\varepsilon\left\|\phi_{R}^{\varepsilon}\right\|_{H^{2}}\right)\left(1+\left\|\phi_{R}^{\varepsilon}\right\|_{L^{2}}\right) .
\end{aligned}
$$

Similar results can be obtained for $\alpha \geq 1$, once we note that $H^{2}$ is an algebra in $\mathbb{R}^{3}$. On the other hand, $R_{\phi}^{\prime \prime}$ depends only on $\phi^{(1)}, \phi^{(2)}$ and $\phi^{(3)},\left\|R_{\phi}^{\prime \prime}\right\|_{H^{k}} \leq C$ for any $0 \leq k \leq s$. Therefore, we arrive at the estimate

$$
\left\|R_{\phi}\right\|_{H^{k}} \leq C\left(\sqrt{\varepsilon}\left\|\phi_{R}^{\varepsilon}\right\|_{H^{\delta}}\right)\left(1+\left\|\phi_{R}^{\varepsilon}\right\|_{H^{k}}\right), \quad \forall 0 \leq k \leq s,
$$

where we have used the fact that a uniform constant $C$ is also of the form $C\left(\sqrt{\varepsilon}\left\|\phi_{R}^{\varepsilon}\right\|_{H^{\delta}}\right)$. Furthermore, if we let $C_{1}(r)=\sup _{0<s<r} C(r)$, the constant $C_{1}(r)$ is nondecreasing. Then (2.22) is proved. The inequality (2.23) can be proved similarly.

Corollary A.1. Let $k \geq 0$ be an integer, then there exists a constant $1 \leq C_{1}=C_{1}\left(\sqrt{\varepsilon}\left\|\phi_{R}^{\varepsilon}\right\|_{H^{\delta}}\right)$, such that

$$
\begin{aligned}
\left\|\bar{\nabla} R_{\phi}\right\|_{H^{k}} & \leq C_{1}\left(\sqrt{\varepsilon}\left\|\phi_{R}^{\varepsilon}\right\|_{H^{\delta}}\right)\left(1+\left\|\bar{\nabla} \phi_{R}^{\varepsilon}\right\|_{H^{k}}\right), \quad \text { and } \\
\left\|\partial_{t} \bar{\nabla} R_{\phi}\right\|_{H^{k}} & \leq C_{1}\left(\sqrt{\varepsilon}\left\|\phi_{R}^{\varepsilon}\right\|_{H^{\delta}}\right)\left(1+\left\|\partial_{t} \bar{\nabla} \phi_{R}^{\varepsilon}\right\|_{H^{k}}\right),
\end{aligned}
$$

where $\delta=\max \{2, k-1\}$. Furthermore, the constant $C_{1}(\cdot)$ can be chosen to be nondecreasing.

\section{B Derivation of the $\mathrm{ZKE}$}

The three dimensional Zakharov-Kuznetsov equation (ZKE) is of the form [17, 23]

$$
\partial_{t} u+u \partial_{x_{1}} u+\partial_{x_{1}} \Delta u=0, \quad x=\left(x_{1}, x_{2}, x_{3}\right) \in \mathbb{R}^{3}, t \in \mathbb{R}
$$

where $\Delta=\partial_{x_{1}}^{2}+\partial_{x_{2}}^{2}+\partial_{x_{3}}^{2}$. In this appendix, we will derive the Zakharov-Kuznetsov equation (ZKE) from the Euler-Poisson system with static magnetic field,

$$
\left\{\begin{array}{l}
\partial_{t} n+\nabla \cdot(n \mathbf{u})=0 \\
\partial_{t} \mathbf{u}+\mathbf{u} \cdot \nabla \mathbf{u}+T_{i} \frac{\nabla n}{n}+\mathbf{e}_{1} \times \mathbf{u}=-\nabla \phi \\
\Delta \phi=e^{\phi}-n,
\end{array}\right.
$$

where $n(t, x), \mathbf{u}(t, x)=\left(u_{1}(t, x), u_{2}(t, x), u_{3}(t, x)\right)$ and $\phi(t, x)$ are respectively the density, velocity of the ions and the electric potential at time $t \geq 0$, position $x=\left(x_{1}, x_{2}, x_{3}\right) \in \mathbb{R}^{3}$. Here $\mathbf{e}_{1}=(1,0,0)^{T}$ is the constant magnetic direction and $T_{i} \geq 0$ is the ion temperature. The formal derivation of the ZKE when $T_{i}=0$ can also be found in [17]. 


\section{B.1 Formal expansion}

Consider the following Gardner-Morikawa transformation in B.1

$$
\varepsilon^{1 / 2}\left(x_{1}-V t\right) \rightarrow x_{1}, \varepsilon^{1 / 2} x_{2} \rightarrow x_{2}, \varepsilon^{1 / 2} x_{3} \rightarrow x_{3}, \varepsilon^{3 / 2} t \rightarrow t .
$$

We obtain the parameterized system

$$
\left\{\begin{array}{l}
\varepsilon \partial_{t} n-V \partial_{x_{1}} n+\nabla \cdot(n \mathbf{u})=0 \\
\varepsilon \partial_{t} \mathbf{u}-V \partial_{x_{1}} \mathbf{u}+\mathbf{u} \cdot \nabla \mathbf{u}+T_{i} \frac{\nabla n}{n}=-\nabla \phi+\frac{1}{\varepsilon^{1 / 2}} \mathbf{u} \times e_{1} \\
\varepsilon \Delta \phi=e^{\phi}-n,
\end{array}\right.
$$

where $\varepsilon$ denotes the amplitude of the initial disturbance and is assumed to be small compared with unity and $V$ is the wave speed to be determined. We consider the following formal expansion

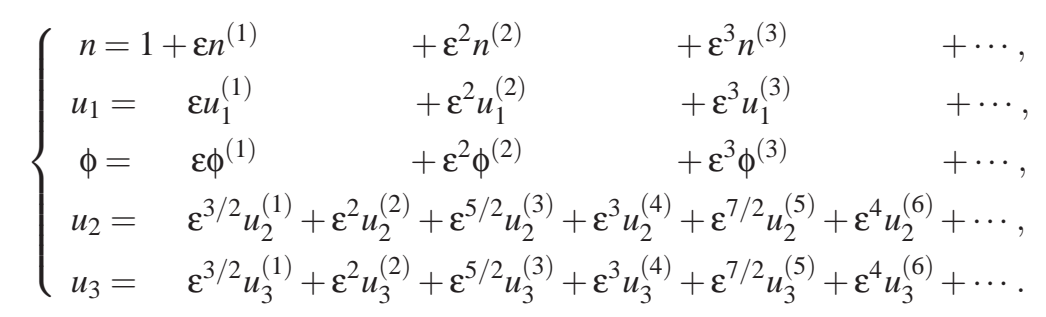

Plugging the formal expansion (B.4) into the system $\underline{B}$, we get a power series of $\varepsilon$, whose coefficients depend on $\left(n^{(k)}, \mathbf{u}^{(k)}, \phi^{(k)}\right)$ for $k \geq 1$.

\section{B.1.1 Derivation of the ZKE for $n^{(1)}$}

At the order of $\varepsilon$, we obtain

Coefficients of $\varepsilon^{1}$ :

$$
\left\{\begin{array}{l}
-V \partial_{x_{1}} n^{(1)}+\partial_{x_{1}} u_{1}^{(1)}=0 \\
V \partial_{x_{1}} u_{1}^{(1)}-T_{i} \partial_{x_{1}} n^{(1)}=\partial_{x_{1}} \phi^{(1)} \\
\phi^{(1)}=n^{(1)} \\
T_{i} \partial_{x_{2}} n^{(1)}=-\partial_{x_{2}} \phi^{(1)}+u_{3}^{(1)} \\
T_{i} \partial_{x_{3}} n^{(1)}=-\partial_{x_{3}} \phi^{(1)}-u_{2}^{(1)}
\end{array}\right.
$$

Consider B.5a B.4c . To get a nontrivial solution, it is necessary to require the determinant of the coefficient matrix of B.5a - B.4c to vanish to obtain

$$
V^{2}=T_{i}+1
$$

For definiteness, we let $V=\sqrt{T_{i}+1}$.

At the orders of $\varepsilon^{3 / 2}$ and $\varepsilon^{2}$, we obtain Coefficients of $\varepsilon^{3 / 2}$ :

$$
\left\{\begin{array}{l}
\partial_{x_{2}} u_{2}^{(1)}+\partial_{x_{3}} u_{3}^{(1)}=0 \\
-V \partial_{x_{1}} u_{2}^{(1)}=u_{3}^{(2)} \\
-V \partial_{x_{1}} u_{3}^{(1)}=-u_{2}^{(2)}
\end{array}\right.
$$


Coefficients of $\varepsilon^{2}$ :

$$
\left\{\begin{array}{l}
\partial_{t} n^{(1)}-V \partial_{x_{1}} n^{(2)}+\partial_{x_{1}}\left(n^{(1)} u_{1}^{(1)}\right)+\partial_{x_{1}} u_{1}^{(2)}+\partial_{x_{2}} u_{2}^{(2)}+\partial_{x_{3}} u_{3}^{(2)}=0 \\
\partial_{t} u_{1}^{(1)}-V \partial_{x_{1}} u_{1}^{(2)}+u_{1}^{(1)} \partial_{x_{1}} u_{1}^{(1)}+T_{i}\left\{\partial_{x_{1}} n^{(2)}-n^{(1)} \partial_{x_{1}} n^{(1)}\right\}=-\partial_{x_{1}} \phi^{(2)} \\
\Delta \phi^{(1)}=\phi^{(2)}+\frac{1}{2}\left(\phi^{(1)}\right)^{2}-n^{(2)} \\
-V \partial_{x_{1}} u_{2}^{(2)}+T_{i}\left\{\partial_{x_{2}} n^{(2)}-n^{(1)} \partial_{x_{2}} n^{(1)}\right\}=-\partial_{x_{2}} \phi^{(2)}+u_{3}^{(3)} \\
-V \partial_{x_{1}} u_{3}^{(2)}+T_{i}\left\{\partial_{x_{3}} n^{(2)}-n^{(1)} \partial_{x_{3}} n^{(1)}\right\}=-\partial_{x_{3}} \phi^{(2)}-u_{2}^{(3)}
\end{array}\right.
$$

From $(\mathrm{B} .5 \mathrm{a})-(\mathrm{B} .5 \mathrm{c})$ and $(\mathrm{B} .6)$, we can assume without loss of generality that

$$
u_{1}^{(1)}=V n^{(1)}, \quad \phi^{(1)}=n^{(1)} .
$$

From (B.5d) and (B.5e), we have

$$
\left\{\begin{array}{l}
u_{2}^{(1)}=-T_{i} \partial_{x_{3}} n^{(1)}-\partial_{x_{3}} \phi^{(1)}=-V^{2} \partial_{x_{3}} n^{(1)} \\
u_{3}^{(1)}=T_{i} \partial_{x_{2}} n^{(1)}+\partial_{x_{2}} \phi^{(1)}=V^{2} \partial_{x_{2}} n^{(1)}
\end{array}\right.
$$

thanks to (B.6) and (B.9). Therefore, to solve $n^{(1)}, \mathbf{u}^{(1)}$ and $\phi^{(1)}$, we need only to solve $n^{(1)}$.

To find out the equation satisfied by $n^{(1)}$, we take $\partial_{x_{1}}$ of $(\mathrm{B} .8 \mathrm{c}$, multiply (B.8a) with $V$, and then add them to $\underline{B} .8 \mathrm{~b}$. We thus obtain

$$
\partial_{t} n^{(1)}+V n^{(1)} \partial_{x_{1}} n^{(1)}+\frac{1}{2 V} \partial_{x_{1}} \Delta n^{(1)}+\frac{1}{2}\left\{\partial_{x_{2}} u_{2}^{(2)}+\partial_{x_{3}} u_{3}^{(2)}\right\}=0 .
$$

On the other hand, from $(\overline{B .7 c}),(B .7 b)$ and $(\bar{B} .10)$, we have

$$
\begin{aligned}
& \partial_{x_{2}} u_{2}^{(2)}=V \partial_{x_{2} x_{1}} u_{3}^{(1)}=V^{3} \partial_{x_{1}} \partial_{x_{2}}^{2} n^{(1)}, \\
& \partial_{x_{3}} u_{3}^{(2)}=-V \partial_{x_{3} x_{1}} u_{2}^{(1)}=V^{3} \partial_{x_{1}} \partial_{x_{3}}^{2} n^{(1)},
\end{aligned}
$$

thanks to (B.6). Inserting this into (B.11), we obtain the Zakarov-Kuznetsov equation

$$
\partial_{t} n^{(1)}+n^{(1)} \partial_{x_{1}} n^{(1)}+\frac{1}{2 V} \partial_{x_{1}}^{3} n^{(1)}+\frac{V^{3}}{2} \partial_{x_{1}} \Delta_{\perp} n^{(1)}=0,
$$

where $\Delta_{\perp}=\partial_{x_{2}}^{2}+\partial_{x_{3}}^{2}$ in $3 \mathrm{D}$.

Proposition B.1. Let $s \geq 9 / 8$, the Cauchy problem of ZKE $\left(\mathbb{B . 1 3}\right.$ is locally well-posed in $H^{s}\left(\mathbb{R}^{3}\right)$.

Proof. See [17].

Remark B.1. B.9), B.10) and (B.13) are a closed system. Once $n^{(1)}$ is solved from B.13, we have all the other first order profiles $\left(\mathbf{u}^{(1)}, \phi^{(1)}\right)$ from $(\mathrm{B} .9)$ and $(\mathrm{B} .10)$. Furthermore, we can also solve $\left(u_{2}^{(2)}, u_{3}^{(2)}\right)$ from $(\mathrm{B} .7 \mathrm{c})$ and $(\mathrm{B} .7 \mathrm{~b})$. In other words, $\left(n^{(1)}, \mathbf{u}^{(1)}, \phi^{(1)}\right)$ and $\left(u_{2}^{(2)}, u_{3}^{(2)}\right)$ can be solved independently, although the equations (B.5), (B.7) and (B.8) for the coefficients of $\varepsilon, \varepsilon^{3 / 2}$ and $\varepsilon^{2}$ depend on the higher order profiles $\left(n^{(2)}, u_{1}^{(2)}, \phi^{(2)}\right)$ and $\left(u_{2}^{(3)}, u_{3}^{(3)}, u_{2}^{(4)}, u_{3}^{(4)}\right)$. 


\section{B.1.2 Derivation of the Linearized ZKE for $n^{(2)}$}

Now, we derive the equation that satisfied by $n^{(2)}$. At the order of $\varepsilon^{5 / 2}$, we obtain Coefficients of $\varepsilon^{5 / 2}$ :

$$
\left\{\begin{array}{l}
\partial_{x_{2}}\left(u_{2}^{(3)}+n^{(1)} u_{2}^{(1)}\right)+\partial_{x_{3}}\left(u_{3}^{(3)}+n^{(1)} u_{3}^{(1)}\right)=0 \\
u_{2}^{(1)} \partial_{x_{2}} u_{1}^{(1)}+u_{3}^{(1)} \partial_{x_{3}} u_{1}^{(1)} \\
\partial_{t} u_{2}^{(1)}-\partial_{x_{1}} u_{2}^{(3)}+u_{1}^{(1)} \partial_{x_{1}} u_{2}^{(1)}=u_{3}^{(4)} \\
\partial_{t} u_{3}^{(1)}-\partial_{x_{1}} u_{3}^{(3)}+u_{1}^{(1)} \partial_{x_{1}} u_{3}^{(1)}=-u_{2}^{(4)}
\end{array}\right.
$$

We first note that $(\overline{B .14 b})$ is consistent with $(\overline{B .5 d})$ and $(B .5 e)$. Indeed, from $(\overline{B .5 d})$ and $(\bar{B} .5 \mathrm{e})$, we can derive $(\bar{B} .14 b)$ by noting $(\bar{B} .9)$. Also, $(\mathrm{B} .14 \mathrm{a})$ is consistent with $(\overline{\mathrm{B} .5 \mathrm{~d}}),(\mathrm{B} .5 \mathrm{e}), \overline{\mathrm{B} .7 \mathrm{~b}},(\mathrm{~B} .7 \mathrm{c}),(\mathrm{B} .8 \mathrm{~d}),(\mathrm{B} .8 \mathrm{e})$. Indeed, from (B.8d) and (B.8e), we have

$$
\begin{aligned}
\partial_{x_{2}} u_{2}^{(3)}+\partial_{x_{3}} u_{3}^{(3)} & =V \partial_{x_{1} x_{2}} u_{3}^{(2)}-V \partial_{x_{1} x_{3}} u_{2}^{(2)} \\
& =-V \partial_{x_{1}}^{2} \partial_{x_{2}} u_{2}^{(1)}-V \partial_{x_{1}}^{2} \partial_{x_{3}} u_{3}^{(1)} \\
& =0,
\end{aligned}
$$

where we have used $(\overline{B .7 b})$ and $(\overline{B .7 c})$ in the second equality and $(\overline{B .5 d})$ and $(\overline{B .5 e})$ in the third equality. Similarly, by using $(\mathrm{B} .5 \mathrm{~d})$ and $(\mathrm{B} .5 \mathrm{e})$, we obtain

$$
\begin{aligned}
\partial_{x_{2}}\left(n^{(1)} u_{2}^{(1)}\right)+\partial_{x_{3}}\left(n^{(1)} u_{3}^{(1)}\right) & =\left\{n^{(1)}\left(\partial_{x_{2}} u_{2}^{(1)}+\partial_{x_{3}} u_{3}^{(1)}\right)\right\}+\left\{u_{2}^{(1)} \partial_{x_{2}} n^{(1)}+u_{3}^{(1)} \partial_{x_{3}} n^{(1)}\right\} \\
& =0 .
\end{aligned}
$$

From (B.14d) and $(\overline{B .8 d})$, we have

$$
\begin{aligned}
u_{2}^{(4)} & =\partial_{x_{1}} u_{3}^{(3)}-\left\{\partial_{t} u_{3}^{(1)}+u_{1}^{(1)} \partial_{x_{1}} u_{3}^{(1)}\right\} \\
& =\partial_{x_{1} x_{2}} \phi^{(2)}+T_{i} \partial_{x_{1} x_{2}} n^{(2)}+{\underline{a_{2}}}^{(1)},
\end{aligned}
$$

where ${\underline{a_{2}}}^{(1)}=-\left\{V \partial_{x_{1}}^{2} u_{2}^{(2)}+\partial_{t} u_{3}^{(1)}+T_{i} \partial_{x_{1}}\left(n^{(1)} \partial_{x_{2}} n^{(1)}\right)+u_{1}^{(1)} \partial_{x_{1}} u_{3}^{(1)}\right\}$. Therefore,

$$
\partial_{x_{2}} u_{2}^{(4)}=\partial_{x_{1}} \partial_{x_{2}}^{2} \phi^{(2)}+T_{i} \partial_{x_{1}} \partial_{x_{2}}^{2} n^{(2)}+\partial_{x_{2}} \underline{a}^{(1)} .
$$

Similarly, from (B.14c) and (B.8e $)$, we obtain

$$
\partial_{x_{3}} u_{3}^{(4)}=\partial_{x_{1}} \partial_{x_{3}}^{2} \phi^{(2)}+T_{i} \partial_{x_{1}} \partial_{x_{3}}^{2} n^{(2)}+\partial_{x_{3}} \underline{a}_{3}{ }^{(1)},
$$

where $\underline{a}_{3}^{(1)}=\left\{\partial_{t} u_{2}^{(1)}+u_{1}^{(1)} \partial_{x_{1}} u_{2}^{(1)}-V \partial_{x_{1}}^{2} u_{3}^{(2)}-T_{i} \partial_{x_{1}}\left(n^{(1)} \partial_{x_{3}} n^{(1)}\right)\right\}$.

At the order of $\varepsilon^{3}$, we obtain 
Coefficients of $\varepsilon^{3}$ :

$$
\left\{\begin{array}{c}
\partial_{t} n^{(2)}-\partial_{x_{1}} n^{(3)}+\partial_{x_{1}}\left(n^{(1)} u_{1}^{(2)}+n^{(2)} u_{1}^{(1)}\right)+\partial_{x_{1}} u_{1}^{(3)}+\partial_{x_{2}} u_{2}^{(4)}+\partial_{x_{3}} u_{3}^{(4)} \\
\quad+\left\{\partial_{x_{2}}\left(n^{(1)} u_{2}^{(2)}\right)+\partial_{x_{3}}\left(n^{(1)} u_{3}^{(2)}\right)\right\}=0 \\
\partial_{t} u_{1}^{(2)}-\partial_{x_{1}} u_{1}^{(3)}+u_{1}^{(1)} \partial_{x_{1}} u_{1}^{(2)}+u_{1}^{(2)} \partial_{x_{1}} u_{1}^{(1)}+T_{i}\left\{\partial_{x_{1}} n^{(3)}-n^{(1)} \partial_{x_{1}} n^{(2)}\right. \\
\left.\quad-\left(n^{(2)}-\left(n^{(1)}\right)^{2}\right) \partial_{x_{1}} n^{(1)}\right\}=-\partial_{x_{1}} \phi^{(3)}+\left\{u_{2}^{(2)} \partial_{x_{2}} u_{1}^{(1)}+u_{3}^{(2)} \partial_{x_{3}} u_{1}^{(1)}\right\} \\
\Delta \phi^{(2)}=\phi^{(3)}+\phi^{(1)} \phi^{(2)}+\frac{1}{3 !}\left(\phi^{(1)}\right)^{3}-n^{(3)} \\
\partial_{t} u_{2}^{(2)}-\partial_{x_{1}} u_{2}^{(4)}+u_{1}^{(1)} \partial_{x_{1}} u_{2}^{(2)}+T_{i}\left\{\partial_{x_{2}} n^{(3)}-n^{(1)} \partial_{x_{2}} n^{(2)}\right. \\
\left.\quad-\left(n^{(2)}-\left(n^{(1)}\right)^{2}\right) \partial_{x_{2}} n^{(1)}\right\}=-\partial_{x_{2}} \phi^{(3)}+u_{3}^{(5)} \\
\partial_{t} u_{3}^{(2)}-\partial_{x_{1}} u_{3}^{(4)}+u_{1}^{(1)} \partial_{x_{1}} u_{3}^{(2)}+T_{i}\left\{\partial_{x_{3}} n^{(3)}-n^{(1)} \partial_{x_{3}} n^{(2)}\right. \\
\left.\quad-\left(n^{(2)}-\left(n^{(1)}\right)^{2}\right) \partial_{x_{3}} n^{(1)}\right\}=-\partial_{x_{3}} \phi^{(3)}-u_{2}^{(5)}
\end{array}\right.
$$

We first note that from $\overline{\mathrm{B} .8 \mathrm{c}}$, we can assume without loss of generality that

$$
\phi^{(2)}=n^{(2)}+\underline{\phi}^{(1)},
$$

where $\underline{\phi}^{(1)}=\Delta \phi^{(1)}-\frac{1}{2}\left(\phi^{(1)}\right)^{2}$ is known from $\left(\overline{B .13}\right.$, since $\phi^{(1)}=n^{(1)}$ from $\overline{B .9}$. From $\overline{\text { B.8a }}$, we have

$$
\partial_{x_{1}} u_{1}^{(2)}=V \partial_{x_{1}} n^{(2)}+\underline{\mathfrak{n}}^{(1)},
$$

where $\underline{\mathfrak{n}}^{(1)}=-\partial_{t} n^{(1)}-\partial_{x_{1}}\left(n^{(1)} u_{1}^{(1)}\right)-\partial_{x_{2}} u_{2}^{(2)}-\partial_{x_{3}} u_{3}^{(2)}$. Without loss of generality, we can assume that

$$
u_{1}^{(2)}=n^{(2)}+\underline{n}^{(1)},
$$

where $\underline{n}^{(1)}=\int_{-\infty}^{x_{1}} \underline{\mathfrak{n}}^{(1)} d x_{1}$.

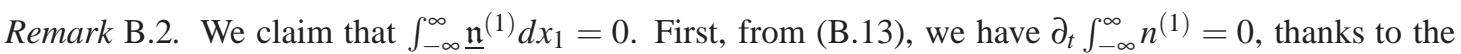
divergence theorem. On the other hand, by $\left(\mathbb{B}\right.$.12 , we have $\underline{\mathfrak{n}}^{(1)}=-\partial_{t} n^{(1)}-\partial_{x_{1}}\left\{\left(n^{(1)} u_{1}^{(1)}\right)+\Delta_{\perp} n^{(1)}\right\}$. The claim then follows, again thanks to divergence theorem.

By taking $\partial_{x_{1}}$ of $(\overline{B .17 \mathrm{c}})$, multiplying $(\mathrm{B} .17 \mathrm{a})$ with $V$ and then adding them to $(\mathrm{B} .17 \mathrm{~b})$, we obtain a linearized inhomogeneous ZKE for $n^{(2)}$ :

$$
\partial_{t} n^{(2)}+\partial_{x_{1}}\left(n^{(1)} n^{(2)}\right)+\frac{1}{2} \partial_{x_{1}}^{3} n^{(2)}+\partial_{x_{1}} \Delta_{\perp} n^{(2)}=\underline{G}^{(1)},
$$

where $\underline{G}^{(1)}$ is the inhomogeneous term, depending only on $n^{(1)}$. Here, we have used (B.15), (B.16), (B.18) and (B.19). Furthermore, we also get the coefficients of $\varepsilon^{7 / 2}$, which depend only on $n^{(2)}$ directly or indirectly:

Coefficients of $\varepsilon^{7 / 2}$ :

$$
\left\{\begin{array}{l}
\partial_{x_{2}}\left(u_{2}^{(5)}+n^{(1)} u_{2}^{(3)}+n^{(2)} u_{2}^{(1)}\right)+\partial_{x_{3}}\left(u_{3}^{(5)}+n^{(1)} u_{3}^{(3)}+n^{(2)} u_{3}^{(1)}\right)=0 \\
u_{2}^{(3)} \partial_{x_{2}} u_{1}^{(1)}+u_{2}^{(1)} \partial_{x_{2}} u_{1}^{(2)}+u_{3}^{(3)} \partial_{x_{3}} u_{1}^{(1)}+u_{3}^{(1)} \partial_{x_{3}} u_{1}^{(2)} \\
\partial_{t} u_{2}^{(3)}-\partial_{x_{1}} u_{2}^{(5)}+\mathbf{u}^{(2)} \nabla u_{2}^{(1)}+u_{1}^{(1)} \partial_{x_{1}} u_{2}^{(3)}+u_{2}^{(1)} \partial_{x_{2}} u_{2}^{(2)}+u_{3}^{(1)} \partial_{x_{3}} u_{2}^{(2)}=u_{3}^{(6)} \\
\partial_{t} u_{3}^{(3)}-\partial_{x_{1}} u_{3}^{(5)}+\mathbf{u}^{(2)} \nabla u_{3}^{(1)}+u_{1}^{(1)} \partial_{x_{1}} u_{3}^{(3)}+u_{2}^{(1)} \partial_{x_{2}} u_{3}^{(2)}+u_{3}^{(1)} \partial_{x_{3}} u_{3}^{(2)}=-u_{2}^{(6)}
\end{array}\right.
$$


Remark B.3 (Continuation of Remark B.1). Once $n^{(2)}$ is solved from B.20), then $u_{1}^{(2)}, \phi^{(2)}, u_{2}^{(3)}, u_{3}^{(3)}, u_{2}^{(4)}$ and $u_{3}^{(4)}$ are all known. Although the expression for the coefficients of $\varepsilon^{3}$ and $\varepsilon^{7 / 2}$ depend on the higher approximations $\left(n^{(3)}, u_{1}^{(3)}, \phi^{(3)}\right)$ and $\left(u_{2}^{(5)}, u_{3}^{(5)}, u_{2}^{(6)}, u_{3}^{(6)}\right)$, they can be solved independently. Furthermore, $n^{(i)}, u_{1}^{(i)}, \phi^{(i)}$ for $i \leq 2$ and $u_{2}^{(j)}$ and $u_{3}^{(j)}$ for $j \leq 4$ will make the systems (B.7), B.8) and (B.14) of the coefficients of $\varepsilon, \varepsilon^{3 / 2}, \varepsilon^{2}, \varepsilon^{5 / 2}$ valid exactly.

\section{B.1.3 The linearized ZKE for $n^{(k)}$}

Inductively, we can derive all the profiles $n^{(k)}, \mathbf{u}^{(k)}$ and $\phi^{(k)} \cdot n^{(k)}$ for $k \geq 3$ satisfy a linearized ZKE similar to B.20

$$
\partial_{t} n^{(k)}+\partial_{x_{1}}\left(n^{(1)} n^{(k)}\right)+\frac{1}{2} \partial_{x_{1}}^{3} n^{(2)}+\partial_{x_{1}} \Delta_{\perp} n^{(k)}=\underline{G}^{(k-1)}
$$

where $\underline{G}^{(k-1)}$ depends only on $n^{(i)}$ for $j \leq k-1$.

Proposition B.2. Let $s \geq 9 / 8$, the Cauchy problem of the linearized inhomogeneous ZKE (B.22) for $k \geq 9 / 8$ is well-posed in $H^{s}\left(\mathbb{R}^{3}\right)$.

Remark B.4 (Continuation of Remark B.3). In particular, we consider the case of $k=3$. Let $n^{(i)}(i=$ $1,2,3)$ be solved from (B.13) and (B.22) for $k=2,3$. Then $u_{1}^{(i)}$ and $\phi^{(i)}$ for $i=1,2,3$ and $u_{2}^{(j)}$ and $u_{3}^{(j)}$ for $j=1, \cdots, 6$ are all known. They will make the systems of the coefficients up to order of $\varepsilon^{7 / 2}$ valid exactly.

\section{B.2 Remainder equation for $\mathrm{ZKE}$}

To make the previous formal derivation rigorous, we consider the following expansion with remainder $\operatorname{term}\left(n_{R}^{\varepsilon}, \mathbf{u}_{R}^{\varepsilon}, \phi_{R}^{\varepsilon}\right)$,

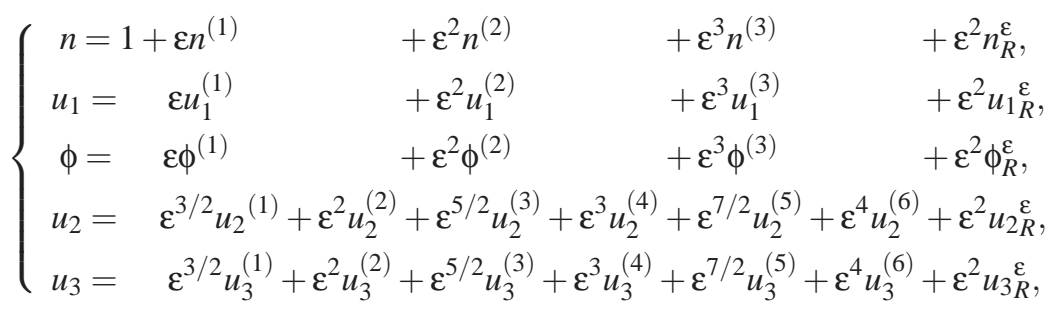

where $\mathbf{u}_{R}^{\varepsilon}=\left(u_{1}^{\varepsilon}, u_{2}^{\varepsilon}, u_{3}^{\varepsilon}\right)$. Here $n^{(1)}, n^{(2)}$ and $n^{(3)}$ satisfy B.13, B.20) and B.22 for $k=3$. The other profiles $u_{1}^{(i)}$ and $\phi^{(i)}$ for $i=1,2,3$ and $u_{2}^{(j)}$ and $u_{3}^{(j)}$ for $j=1, \cdots, 6$ are solved from the systems ( $\mathrm{B} .5$, (B.7), (B.8), (B.14), B.17) and (B.21) of coefficients up to order $\varepsilon^{7 / 2}$. See Remark B.1, B.3 and B.4

For notational convenience, we denote $\mathbf{u}=\left(u_{1}, u_{2}, u_{3}\right)^{T}, \mathbf{u}_{R}^{\varepsilon}=\left(u_{1}^{\varepsilon}, u_{2}^{\varepsilon}, u_{3}^{\varepsilon}\right)^{T}$ and

$$
\begin{aligned}
\widetilde{n} & =n^{(1)}+\varepsilon n^{(2)}+\varepsilon^{2} n^{(3)}, \quad \widetilde{\phi}=\phi^{(1)}+\varepsilon \phi^{(2)}+\varepsilon^{2} \phi^{(3)}, \\
\widetilde{\mathbf{u}} & =\left(\widetilde{u}_{1}, \widetilde{u}_{2}, \widetilde{u}_{3}\right)^{T}, \quad \widetilde{u}_{1}=u_{1}^{(1)}+\varepsilon u_{1}^{(2)}+\varepsilon^{2} u_{1}^{(3)}, \\
\widetilde{u}_{2} & =\varepsilon^{1 / 2} u_{2}^{(1)}+\varepsilon u_{2}^{(2)}+\varepsilon^{3 / 2} u_{2}^{(3)}+\varepsilon^{2} u_{2}^{(4)}+\varepsilon^{5 / 2} u_{2}^{(5)}+\varepsilon^{3} u_{2}^{(6)}, \\
\widetilde{u}_{3} & =\varepsilon^{1 / 2} u_{3}^{(1)}+\varepsilon u_{3}^{(2)}+\varepsilon^{3 / 2} u_{3}^{(3)}+\varepsilon^{2} u_{3}^{(4)}+\varepsilon^{5 / 2} u_{3}^{(5)}+\varepsilon^{3} u_{3}^{(6)} .
\end{aligned}
$$


Proposition B.3. Let $(n, \boldsymbol{u}, \phi)$ in (B.23) be a solution of the Euler-Poisson system (B.1), then $\left(n_{R}^{\varepsilon}, \boldsymbol{u}_{R}^{\varepsilon}, \phi_{R}^{\varepsilon}\right)$ satisfy the following remainder system

$$
\left\{\begin{aligned}
\partial_{t} n_{R}^{\varepsilon}- & \frac{V \boldsymbol{e}_{1}-\boldsymbol{u}}{\varepsilon} \cdot \nabla n_{R}^{\varepsilon}+\frac{n}{\varepsilon} \nabla \cdot \boldsymbol{u}_{R}^{\varepsilon}+n_{R}^{\varepsilon} \nabla \cdot \widetilde{\boldsymbol{u}}+\boldsymbol{u}_{R}^{\varepsilon} \cdot \nabla \widetilde{n}+\varepsilon R_{n}=0 \\
\partial_{t} \boldsymbol{u}_{R}^{\varepsilon}- & \frac{V \boldsymbol{e}_{1}-\boldsymbol{u}}{\varepsilon} \cdot \nabla \boldsymbol{u}_{R}^{\varepsilon}+\boldsymbol{u}_{R}^{\varepsilon} \nabla \cdot \widetilde{\boldsymbol{u}}+\frac{T_{i}}{\varepsilon n} \bar{\nabla} n_{R}^{\varepsilon} \\
& \quad-\frac{T_{i} \boldsymbol{p}}{\varepsilon n} n_{R}^{\varepsilon}-\frac{T_{i} \varepsilon}{n} \boldsymbol{R}_{T}+\varepsilon \boldsymbol{R}_{\boldsymbol{u}}=-\frac{1}{\varepsilon} \nabla \phi_{R}^{\varepsilon}+\frac{1}{\varepsilon^{3 / 2}} \boldsymbol{u}_{R}^{\varepsilon} \times \boldsymbol{e}_{1}, \\
\varepsilon \Delta \phi_{R}^{\varepsilon}= & \phi_{R}^{\varepsilon}-n_{R}^{\varepsilon}+\varepsilon \phi^{(1)} \phi_{R}^{\varepsilon}+\varepsilon^{3 / 2} R_{\phi}
\end{aligned}\right.
$$

where $\boldsymbol{u}_{R}^{\varepsilon}=\left(u_{1}^{\varepsilon}, u_{2}^{\varepsilon}, u_{3}^{\varepsilon}\right)$ and $\widetilde{n}, \widetilde{\boldsymbol{u}}$ and $\widetilde{\phi}$ are given in (B.24). Here, $R_{n}, \boldsymbol{R}_{\boldsymbol{u}}=\left(R_{\boldsymbol{u} 1}, R_{\boldsymbol{u} 2}, R_{\boldsymbol{u} 3}\right)$ depend only on $n^{(k)}, \boldsymbol{u}^{(k)}$ and $\phi^{(k)}$, and $R_{\phi}$ depends on $\phi_{R}^{\varepsilon}$ in the form $R_{\phi}=F\left(\sqrt{\varepsilon} \phi_{R}^{\varepsilon}\right) \phi_{R}^{\varepsilon}+\sqrt{\varepsilon} R_{\phi}^{\prime}$ for some $R_{\phi}^{\prime}$ depending only on $n^{(k)}, \boldsymbol{u}^{(k)}$ and $\phi^{(k)}$. In $\mathrm{B} .25 \mathrm{~b}, \boldsymbol{p}=\left(p_{1}, p_{2}, p_{3}\right)$ and $\boldsymbol{R}_{T}=\left(R_{T 1}, R_{T 2}, R_{T 3}\right)$ are finite combinations of $n^{(1)}, n^{(2)}$ and $n^{(3)}$. In $\mathrm{B} .25 \mathrm{a}, \boldsymbol{e}_{1}=(1,0,0)^{\prime}$ is a constant vector.

The derivation of such a system for $\left(n_{R}^{\varepsilon}, \mathbf{u}_{R}^{\varepsilon}, \phi_{R}^{\varepsilon}\right)$ is similar to that of 2.21 in the KPE limit case. (B.25c) can also be written in the equivalent form of 2.29) in Proposition 2.4. The remainder term $R_{\phi}$ and $\bar{R}_{\phi}$ satisfy the same estimates of Lemma2.1. These claims can be proved exactly as those in Appendix A and hence omitted.

\section{Commutator estimates}

We give two important inequalities which are widely used throughout this paper [14].

Lemma C.1. Let $\alpha$ be any multi-index with $|\alpha|=k$ and $p \in(1, \infty)$. Then there exists some constant $C>0$ such that

$$
\begin{aligned}
&\left\|\partial_{x}^{\alpha}(f g)\right\|_{L^{p}} \leq C\left\{\|f\|_{L^{p_{1}}}\|g\|_{\dot{H}^{s, p_{2}}}+\|f\|_{\dot{H}^{s, p_{3}}}\|g\|_{L^{p_{4}}}\right\}, \\
&\left\|\left[\partial_{x}^{\alpha}, f\right] g\right\|_{L^{p}} \leq C\left\{\|\nabla f\|_{L^{p_{1}}}\|g\|_{\dot{H}^{k-1, p_{2}}}+\|f\|_{\dot{H}^{k, p_{3}}}\|g\|_{L^{p_{4}}}\right\},
\end{aligned}
$$

where $f, g \in \mathscr{S}$, the Schwartz class and $p_{2}, p_{3} \in(1,+\infty)$ such that

$$
\frac{1}{p}=\frac{1}{p_{1}}+\frac{1}{p_{2}}=\frac{1}{p_{3}}+\frac{1}{p_{4}} \text {. }
$$

Acknowledgment This research is supported by NSFC under grant 11001285 . The author thanks B. Pausader for pointing out the problem, helpful discussions and encouragement.

\section{References}

[1] J. Bourgain, On the Cauchy problem for the Kadomstev-Petviashvili equation, Geom. Funct. Anal., 3(4), (1993)315-341.

[2] D. Chiron and F. Rousset, The KdV/KP-I limit of the nonlinear Schrodinger equation, SIAM J. Math. Anal., 42(1), (2010)64-96.

[3] S. Cordier and E. Grenier, Quasineutral limit of an Euler-Poisson system arising from plasma physics, Commun. Partial Differential Equations, 25(5\&6), (2000)1099-1113.

[4] S. Engelberg, H. Liu, E. Tadmor, Critical thresholds in Euler-Poisson equations, Indiana Univ. Math. J., 50, (2001)109-257.

[5] E. Grenier, Pseudo-differential energy estimates of singular perturbations, Comm. Pure Appl. Math., 50(9), (1997)0821-0865. 
[6] E. Grenier, Y. Guo and B. Pausader, Derivation of the ion equation, In preparation, 2011.

[7] Y. Guo, Smooth irrotational flows in the large to the Euler-Poisson system in $R^{3+1}$, Commun. Math. Phys., 195, (1998)249-265.

[8] Y. Guo and J. Jang, Global Hilbert expansion for the Vlasov-Poisson-Boltzmann system, Commun. Math. Phys., 299, (2010)469-501.

[9] Y. Guo and B. Pausader, Global smooth ion dynamics in the Euler-Poisson system, Commun. Math. Phys., 303, (2011)89-125.

[10] Y. Guo and X. Pu, KdV limit of the Euler-Poisson equations, submitted.

[11] M. Hadac, S. Herr and H. Koch, Well-posedness and scattering for the KP-II equation in a critical space, Ann. Inst. H. Poincaré Anal. Non Linéaire, 26, (2009)917-941

[12] P. Isaza, J. Lopez and J. Mejia, The Cauchy problem for the Kadomtsev-Petviashbili (KPII) equation in three space dimensions, Comm. Partial Differential Equations, 32, (2007)611-641.

[13] B.B. Kadomtsev and V. I. Petviashvili, On the stability of solitary waves in weakly dispersive media, Soviet Phys. Dokl. 15, (1970)539-541.

[14] T. Kato and G. Ponce, Commutator estimates and the Euler and Navier-Stokes equations, Comm. Pure Appl. Math., 41, 891-907(1988)

[15] D.J. Korteweg and G. de Vries, On the change of form of long waves advancing in a rectangular channel, and on a new type of long stationary waves, Phil. Mag., 39, (1985)422-443.

[16] H.L. Li, A. Matsumura, G. Zhang, Optimal decay rate of the compressible Navier-Stokes-Poisson system in $\mathbb{R}^{3}$. Arch. Ration. Mech. Anal. 196(2), (2010)681-713.

[17] F. Linares and J.C. Saut, The Cauchy problem for the 3D Zakharov-Kuznetsov equation, Discret. Contin. Dyn. Syst., 24(2), (2009)547-565.

[18] H. Liu, E. Tadmor, Spectral dynamics of the velocity gradient field in restricted flows. Commun. Math. Phys. 228(3), (2002)435-466.

[19] H. Liu, E. Tadmor, Critical thresholds in 2D restricted Euler-Poisson equations. SIAM J. Appl. Math. 63(6), (2003)1889-1910.

[20] L. Molinet, J. Saut and N. Tzvetkov, Global well-posedness for the KP-II equation on the background of a non-localized solution, Ann. Inst. H. Poincaré Anal. Non Linéaire, 28, (2011)653-676.

[21] G. Schneider, C.E. Wayne, The long-wave limit for the water wave problem I. The case of zero surface tension, Comm. Pure Appl. Math., 53(12), (2000)1475-1535.

[22] E.M. Stein, Singular integrals and differentiability properties of functions, Princeton University Press, Princeton, New Jersey, 1970.

[23] V. E. Zakharov and E. A. Kuznetzov, On three dimensional solitons, Sov. Phys. JETP., 39(1974), 285-286. 